\title{
EXTINCTION TIME FOR THE WEAKER OF TWO COMPETING SIS EPIDEMICS
}

\author{
FABIO LOPES AND MALWINA LUCZAK
}

\begin{abstract}
We consider a simple stochastic model for the spread of a disease caused by two virus strains in a closed homogeneously mixing population of size N. The spread of each strain in the absence of the other one is described by the stochastic logistic SIS epidemic process, and we assume that there is perfect cross-immunity between the two strains, that is, individuals infected by one are temporarily immune to re-infections and infections by the other. For the case where one strain has a strictly larger basic reproductive ratio than the other, and the stronger strain on its own is supercritical (that is, its basic reproductive ratio is larger than 1), we derive precise asymptotic results for the distribution of the time when the weaker strain disappears from the population, that is, its extinction time. We further extend our results to certain parameter values where the difference between the two reproductive ratios may tend to 0 as $N \rightarrow \infty$.

In proving our results, we illustrate a new approach to a fluid limit approximation for a sequence of Markov chains in the vicinity of a stable fixed point of the limit.
\end{abstract}

\section{INTRODUCTION}

Mathematical models of epidemics provide important tools for understanding the spread of many diseases relevant to public health, and may help health authorities and organizations develop measures to prevent and manage epidemic outbreaks, as well as control the emergence of new infections.

Infectious disease control is constantly challenged by the diversity of pathogen populations and their continuous evolution in response to changing environments, technological advances (e.g. air travel, antibiotics, see Schrag and Wiener 1995), interactions with their hosts (e.g. the structure of the network of contacts, see Leventhal et al. 2015), as well as interactions with other pathogens (e.g. interference, see Gart and De Vries 1966).

2000 Mathematics Subject Classification. 60J27, 92D30.

Key words and phrases. stochastic SIS logistic epidemic; competing SIS epidemics; time to extinction; near-critical epidemic.

FL was first a postdoctoral research assistant of ML funded by EPSRC Leadership Fellowship EP/J004022/2, and was later supported partly by Conicyt/Fondecyt Proyecto Postdoctorado N.3160163.

ML was supported partly by EPSRC Leadership Fellowship EP/J004022/2 and partly by ARC Future Fellowship FT170100409. 
The vast majority of mathematical models of epidemics in the literature view infectious diseases as caused by a single and stable pathogen strain. Such models tend to be more tractable, and may even yield accurate descriptions of the short-term dynamics of certain diseases. However, they may be inappropriate for predicting the long-term evolutionary dynamics of pathogen populations, see Humplik et al. (2014), or for analysing pathogen infections where host susceptibility may be altered due to infections by other pathogens. Examples include interference between co-morbid diseases (e.g. yaws and chickenpox, see Gart and De Vries 1966), as well as scenarios where successive exposures to different strains of the pathogen may have important consequences for disease infectiousness and severity (e.g. dengue fever, see Feng and Velasco-Hernandez 1997).

Pathogen evolution and the capacity of pathogens to adapt to changes in their environment and hosts are both regarded as important factors for emergence of new diseases (Antia et al. 2003; Arinaminpathy and McLean 2009). For instance, it is known that pathogen strains which are sufficiently antigenically similar may induce a (partial) cross-protective immune response, so that hosts infected by one of the strains may acquire different degrees of temporary or permanent immunity to re-infections and infections by antigenically similar strains. Thus, if a certain closed population of hosts is affected by a particular virus strain and a number of individuals infected by an antigenically similar strain are introduced, then the different pathogen strains may interact as if competing for susceptible individuals in the host population.

A long-standing principle in ecology known as the competitive exclusion principle (Levin 1970) predicts that, when species sharing the same ecological niche compete for limited resources, then the one with even the slightest advantage will eventually outcompete the others and become dominant. This form of competition is believed to be particularly important in the evolution of RNA viruses (Domingo et al. 1996; Moya et al. 2004). For instance, it is shown by Bahl et al. (2009) that viral gene flow from Eurasia had led to replacement of endemic avian influenza viruses in North America; moreover, the authors argue that the most likely mechanism for that was competition for susceptible hosts.

A number of deterministic models of coexistence and competitive exclusion in multiple strains pathogen populations have been studied, describing the dynamics of important diseases such as gonorrhea (Castillo-Chavez et al. 1999), AIDS (Anderson and May 1996), and influenza (Andreasen et al. 1997); see also references within these works. However, deterministic models often fail to provide accurate descriptions of infectious disease dynamics at the beginning or at the end of an outbreak; this is partly because random fluctuations when the number of infectives is small can significantly affect the outcome, in particular, the chances of the disease persisting in the population, as well as the duration of the outbreak. 
In this work, we consider a simple model for the spread of a disease with stochastic susceptible-infective-susceptible (SIS) dynamics caused by two different virus strains with a perfect cross-protective immune response, so individuals infected by one strain are temporarily immune to re-infections and infections by the other strain. We focus on the case where one of the virus strains has some advantage over its competitor (a higher basic reproductive ratio), and competitive exclusion occurs. Starting with positive but otherwise arbitrary proportions of infected individuals of each virus strain in a large host population, we track the long-term evolution of this process, so as to obtain the distribution of the time until competitive exclusion occurs, that is the extinction time of the weaker virus strain.

The simplest stochastic model for a disease with SIS dynamics is the stochastic SIS logistic epidemic model. In that model, each individual within the population is either susceptible or infective. We assume a population of size $N$, and let $\lambda>0$ denote the infection rate. Each infective individual encounters uniformly at random another member of the population at rate $\lambda$; if the encountered individual is susceptible, then he/she becomes infective. Also, each infective individual recovers at rate $\mu>0$ and, once recovered, becomes susceptible again.

Let $Y_{N}(t)$ denote the number of infective individuals in the population at time $t$; then $\left(Y_{N}(t)\right)_{t \geq 0}$ is a continuous-time Markov chain on $\{0,1, \ldots, N\}$ with transition rates from state $Y$ given by

$$
\begin{array}{ll}
Y \rightarrow Y+1 & \text { at rate } \lambda Y(1-Y / N) ; \\
Y \rightarrow Y-1 & \text { at rate } \mu Y .
\end{array}
$$

The extinction time $\tau_{N}$ is defined as $\tau_{N}=\inf \left\{t \geq 0: Y_{N}(t)=0\right\}$, and, since the state space is finite, $\tau_{N}$ is a.s. finite. The following theorem summarises asymptotic results for the distribution of $\tau_{N}$ in the case where the initial epidemic infects a positive proportion of the population, see Andersson and Djehiche (1998), as well as Brightwell, House, and Luczak (2018).

We recall that a random variable $W$ has a standard Gumbel distribution if $\mathbb{P}(W \leq w)=e^{-e^{-w}}$, for all $w \in \mathbb{R}$.

Theorem 1. Let $\lambda, \mu, \alpha>0$, and suppose that $X_{N}(0) / N \rightarrow \alpha$ as $N \rightarrow \infty$.

(i) (Supercritical case; Andersson and Djehiche (1998).) If $\lambda>\mu$, then $\tau_{N} / \mathbb{E}\left(\tau_{N}\right) \rightarrow Z$ in distribution, as $N \rightarrow \infty$, where $Z$ is an exponential random variable with parameter 1 . Furthermore,

$$
\mathbb{E}\left(\tau_{N}\right) \sim \sqrt{\frac{2 \pi}{N}} \frac{\lambda}{(\lambda-\mu)^{2}} e^{N v},
$$

as $N \rightarrow \infty$, where $v=\log (\lambda / \mu)-1+\frac{\mu}{\lambda}$.

(ii) (Subcritical case; Brightwell, House and Luczak (2018).) If $\lambda<\mu$, then as $N \rightarrow \infty$

$(\mu-\lambda) \tau_{N}-\{\log \alpha+\log N+\log (1-\lambda / \mu)-\log (1+\lambda \alpha /(\mu-\lambda))\} \rightarrow W$ in distribution, where $W$ is a standard Gumbel random variable. 
When $\lambda=\mu$, then, for most starting states, the time to extinction is of the order $N^{1 / 2}$, see Nasell (2011). Brightwell, House and Luczak (2018) also consider more general initial conditions, as well as determine the extinction time when $\lambda=\lambda(N), \mu=\mu(N)$ satisfy $\mu-\lambda \rightarrow 0$ and $(\mu-\lambda) N^{1 / 2} \rightarrow \infty$ (the barely subcritical case).

The stochastic SIS logistic competition model describes the spread of a disease in a homogeneously mixing population via two different virus strains, say types 1 and 2, which are sufficiently antigenically similar to induce a cross-protective immune response. An individual infected with strain $i$ ( $i=$ $1,2)$ stays infected for an exponentially distributed time with rate $\mu_{i}>0$, and, during the infectious period, they independently make an infectious contact to a random individual according to a Poisson process with rate $\lambda_{i}>$ 0 ; if the individual is currently susceptible, then they become infected with strain $i$ as a result. The dynamics can thus be described as a two-dimensional continuous-time Markov chain $\left(X_{N}(t)\right)_{t \geq 0}=\left(X_{N, 1}(t), X_{N, 2}(t)\right)_{t \geq 0}$, where $X_{N, 1}(t)$ and $X_{N, 2}(t)$ denote the numbers of individuals infected with strains of type 1 and 2 respectively, at time $t$. The state space is $S_{N}=\left\{\left(X_{1}, X_{2}\right)^{T}\right.$ : $\left.X_{1}, X_{2} \in \mathbb{Z}^{+}, 0 \leq X_{1}+X_{2} \leq N\right\}$, and the transition rates from state $\left(X_{1}, X_{2}\right)$ can be written as follows:

$$
\begin{array}{llll}
\left(X_{1}, X_{2}\right) \rightarrow\left(X_{1}+1, X_{2}\right) & \text { at rate } & \lambda_{1} X_{1}\left(1-X_{1} / N-X_{2} / N\right) ; \\
\left(X_{1}, X_{2}\right) \rightarrow\left(X_{1}, X_{2}+1\right) & \text { at rate } & \lambda_{2} X_{2}\left(1-X_{1} / N-X_{2} / N\right) ; \\
\left(X_{1}, X_{2}\right) \rightarrow\left(X_{1}-1, X_{2}\right) & \text { at rate } & \mu_{1} X_{1} ; \\
\left(X_{1}, X_{2}\right) \rightarrow\left(X_{1}, X_{2}-1\right) & \text { at rate } & \mu_{2} X_{2} .
\end{array}
$$

We note that, in the absence of one of the strains, the other strain evolves according to the basic stochastic SIS logistic epidemic model described above.

We assume that $\lambda_{1} / \mu_{1}>\lambda_{2} / \mu_{2}$, and $\lambda_{1} / \mu_{1}>1$, as well as that $X_{N, 1}(0) /$ $N \rightarrow \alpha$ and $X_{N, 2}(0) / N \rightarrow \beta$ as $N \rightarrow \infty(0<\alpha, \beta, \alpha+\beta \leq 1)$. The assumption $\lambda_{1} / \mu_{1}>\lambda_{2} / \mu_{2}$ means that strain 1 has a higher basic reproductive ratio, and is thus more infectious than strain 2 . Since $\lambda_{1} / \mu_{1}>1$, Theorem 1 implies that the stronger subtype, in the absence of its competitor, would stay endemic in the population for a time that grows exponentially in the size $N$ of the population.

This model was proposed by Parsons and Quince (2007a,b) as an extension to the Moran model for a haploid population studied in Moran (1958). They assume that both alleles (strains) are supercritical, which in our setting translates to assuming that $\lambda_{2} / \mu_{2}>1$. Parsons and Quince (2007a) consider the case where one of alleles (strains) is weaker (the case considered in the present paper), while Parsons and Quince (2007b) consider the case where both types of allele (strain) have equal fitness, translating to the case $\lambda_{1} / \mu_{1}=\lambda_{2} / \mu_{2}$, not studied here. Parsons and Quince (2007a,b) study the allele fixation probability for this model, equivalent to the probability of one virus strain displacing the other in the competing epidemic setting. Also, Humplik et al. (2014) study the effects of virulence on the probability of 
strain 2 invading strain 1, mainly for small populations, though they appear to be unaware of the earlier works of Parsons and Quince (2007a,b).

For a closely related model with $K \geq 2$ types, Parsons, Quince and Plotkin (2008) obtain analytic approximations for the expected fixation time (i.e. the time until competitive exclusion occurs), which turns out to be linear in the population size when all the alleles (strains) have the same (supercritical) basic reproductive ratio. These authors further argue that a similar result should hold for the model considered in our paper, and Kogan et al. (2014) have shown this is indeed the case for $K=2$ strains of equal strength.

Theorem 2 below concerns the case where there is a dominant, supercritical, strain and each of the two strains initially affects a positive fraction of the population. Under these conditions, competitive exclusion of the weaker strain by the stronger occurs with high probability (i.e. with probability tending to 1 as the population size $N \rightarrow \infty$ ). Our result shows that, with high probability, the extinction time for the weaker type scales logarithmically while the time to extinction for the dominant strain scales exponentially with the population size.

The corresponding deterministic SIS logistic competition model is among the simplest epidemic models for infections caused by multiple pathogen strains. It is represented by the pair

$$
\begin{aligned}
& \frac{d x_{1}(t)}{d t}=\lambda_{1} x_{1}(t)\left(1-x_{1}(t)-x_{2}(t)\right)-\mu_{1} x_{1}(t) \\
& \frac{d x_{2}(t)}{d t}=\lambda_{2} x_{2}(t)\left(1-x_{1}(t)-x_{2}(t)\right)-\mu_{2} x_{2}(t)
\end{aligned}
$$

of differential equations, and is thus a particular instance of the deterministic Lotka-Volterra system - see Lotka (1925), Volterra (1931), Zeeman (1995) and Chapter 8 of Renshaw (2011) - which has found applications, for instance, in biology, ecology, and economics. Various stochastic LotkaVolterra systems have also been studied. For instance, Kirupaharan and Allen (2004) study a stochastic Lotka-Volterra system with multiple species and demography (i. e. births and deaths), with a focus on the probability distributions of the numbers of individuals of each species conditioned on non-extinction. They provide numerical examples of competitive exclusion as well as coexistence, and compare the behaviour of the stochastic model to its deterministic version. Also, Cattiaux and Méléard (2010) consider a stochastic Lotka-Volterra process on $\mathbb{R}_{+}^{2}$, modeling interactions in a two-type density dependent population as a generalisation of the one-dimensional logistic Feller diffusion. They study the long-term behaviour of the process, proving existence and uniqueness of the quasi-stationary distribution in different regions of the parameter space. For the parameter region in which the two types compete, they show that there is a timescale on which only one type survives, though they do not consider the distribution of the time until competitive exclusion occurs. 
Let $R_{0,1}=\lambda_{1} / \mu_{1}$ and $R_{0,2}=\lambda_{2} / \mu_{2}$ denote the basic reproductive ratios of the two strains. $\kappa_{N}=\inf \left\{t \geq 0: X_{N, 2}(t)=0\right\}$, the time when the weaker competing species goes extinct. Let also $\tau_{N}=\inf \left\{t \geq 0: X_{N, 1}(t)=0\right\}$, the time the stronger species becomes extinct. We now state our main result, concerning the distribution of $\kappa_{N}, \tau_{N}$.

Theorem 2. Suppose that $R_{0,1}>R_{0,2}$ and that $R_{0,1}>1$. Suppose further that $X_{N, 1}(0) / N \rightarrow \alpha$ and $X_{N, 2}(0) / N \rightarrow \beta$ as $N \rightarrow \infty$, where $\alpha, \beta>0$ and $\alpha+\beta \leq 1$. Then, as $N \rightarrow \infty$,

$$
\mu_{2}\left(1-\frac{R_{0,2}}{R_{0,1}}\right) \kappa_{N}-\left[\log \left(N \beta\left(1-\frac{R_{0,2}}{R_{0,1}}\right)\right)+\frac{R_{0,2} \mu_{2}}{R_{0,1} \mu_{1}} \log \left(\frac{1-R_{0,1}^{-1}}{\alpha}\right)\right] \rightarrow W,
$$

where $W$ has a standard Gumbel distribution.

Furthermore, as $N \rightarrow \infty$,

$$
\mathbb{E}\left(\tau_{N}\right) \sim \sqrt{\frac{2 \pi}{N}} \frac{\lambda_{1}}{\left(\lambda_{1}-\mu_{1}\right)^{2}} e^{N v_{1}}
$$

as $N \rightarrow \infty$, where $v_{1}=\log \left(\lambda_{1} / \mu_{1}\right)-1+\frac{\mu_{1}}{\lambda_{1}}$, and $\tau_{N} / \mathbb{E}\left(\tau_{N}\right) \rightarrow Z$ in distribution, where $Z$ is an exponential random variable with parameter 1.

Theorem 2 thus shows that the extinction time $\kappa_{N}$ of the weaker strain can be written as

$$
\kappa_{N}=\frac{\log N+Z}{\mu_{2}\left(1-\frac{R_{0,2}}{R_{0,1}}\right)},
$$

where $Z$ is a random variable with a bounded mean and variance, while the extinction time $\tau_{N}$ of the stronger strain asymptotically has the same distribution as if the weaker strain was absent to begin with.

The long-term behaviour of Markov population processes is of considerable importance in applications. In epidemic models, long-term phenomena include extinction of certain pathogen strains, or replacement of a dominant pathogen strain in the host population by another more adapted pathogen strain introduced into the host population e.g. due to mutation or migration. Mathematically, these phenomena are related to the behaviour of the scaled process near fixed points of its approximating differential equation, including absorbing boundaries for one or more coordinates. Recently, Barbour, Hamza, Kaspi and Klebaner (2015) have shown that, under appropriate conditions, a density dependent Markov population process that starts near an absorbing boundary and manages to escape from it, still can be wellapproximated by the deterministic solution as described by the standard theory but with a random time shift, and that the time to escape from such a boundary is random and of order $O(\log N)$, see Theorem 1.1 in Barbour et al. (2015). Also, similar to the phenomenon we investigate in the present work, they describe in a very general setting the behaviour of a class of population processes near a fixed point at which one or more coordinates of the process have value 0 , i.e. they are extinct, and derive the limit distribution for the extinction times for such processes as a standard Gumbel 
random variable, after scaling and centering, see Theorem 1.2 in Barbour et al. (2015). In both results, the randomness when the process is escaping or reaching an absorbing boundary is captured by a branching process approximation. However, at their level of generality, the formulae they obtain contain non-explicit constants, and their bounds on the rate of convergence are too weak to investigate near-critical phenomena. Also, rigorous justification of such a general approximation, based on an abstract coupling of Thorisson (see Theorem 7.3 in Thorisson 2000) is quite involved.

In the present work, we develop a related but more direct and optimal approximation to prove an explicit formula for the extinction time of the weaker virus strain in the stochastic logistic SIS competition model. Like the approach of Barbour et al. (2015), our approach is based on decomposing the drifts of the process into linear and non-linear parts, and using a variation of constants formula. However, we additionally take full advantage of the fact that the non-linear parts are small in the neighbourhood of a fixed point, and provide more refined bounds on the deviations of the martingale transform appearing in the equations. Similar ideas were also used in a different context by Barbour and Luczak (2012) and, in discrete time, by Brightwell and Luczak (2012).

Unlike the approach of Barbour et al. (2015), the precision of our approximation facilitates study of near-critical phenomena. In Section 7, we allow the basic reproductive ratios $R_{0,1}$ and $R_{0,2}$ to be functions of the population size $N$. We show that Theorem 2 below can be extended to certain near-critical regimes where $\lambda_{1} / \mu_{1}-\lambda_{2} / \mu_{2} \rightarrow 0$, while $\lambda_{1} / \mu_{1}$ may or may not tend to 1 as $N \rightarrow \infty$. We do not cover the entire spectrum of near-critical behaviours: the example considered here is meant as a proof of concept, and a full investigation will be carried out systematically in future work. One challenge of such an investigation will be to understand the behaviour of the approximating deterministic process in various near-critical regimes. Also in future work we intend to study the critical case when the strengths of the two strains are even closer to identical, and to extend our results to competition of more than 2 strains. A further project is to rigorously study the probability that the strongest strain wins when starting with only a small number of infected individuals relative to the number of infectives with weaker strains, in particular in near-critical scenarios, where there is likely to be a delicate interplay between initial conditions and the asymptotic differences between the strengths of the different strains.

In Section 2, we present some preliminaries concerning the stability of fixed points of the deterministic logistic SIS competition model. Furthermore, we give an overview of the strategy used to prove Theorem 2 , The idea is that the stochastic logistic SIS competition process follows closely the corresponding deterministic process for a long time, until the latter one is close to its attractive fixed point at $\left(\left(\lambda_{1}-\mu_{1}\right) / \lambda_{1}, 0\right)^{T}$. From there on, the time to extinction for the second species is short, and well approximated by a linear birth-and-death chain, with the randomness captured by the 
Gumbel distribution. We break up the analysis of the process into phases, similarly to the approach of Brightwell, House and Luczak (2018) used to prove a general version of Theorem 1 $(i i)$. We analyze each of these phases in the subsequent sections. In Section 6, we combine the results obtained in the preceding sections to prove Theorem 2.

In Section 7, we state and prove Theorem 4, which extends our work to a near-critical case where $R_{0,1}=R_{0,1}(N)$ and $R_{0,2}=R_{0,2}(N)$ are such that $R_{0,1}-R_{0,2} \rightarrow 0$, while $R_{0,1}-1$ may or may not tend to 0 but satisfies $\left(R_{0,1}-R_{0,2}\right)\left(R_{0,1}-1\right)^{-1} \rightarrow 0$ as $N \rightarrow \infty$. In the particular case we consider, $\mu_{1}=\mu_{2}=1$, and $\lambda_{1}=\lambda_{1}(N), \lambda_{2}=\lambda_{2}(N)$ are such that $\left(\lambda_{1}-\lambda_{2}\right)\left(\lambda_{1}-\right.$ $1)^{-1} \rightarrow 0$. We further assume that

$$
N\left(\lambda_{1}-\lambda_{2}\right)^{3}\left(\lambda_{1}-1\right)^{-1} / \log \log \left(N\left(\lambda_{1}-\lambda_{2}\right)^{2}\right) \rightarrow \infty .
$$

Condition 2 is an artefact of our proof technique, and does not define the transition to criticality. We do believe that, similarly to a single stochastic SIS logistic epidemic, the condition $N\left(\lambda_{1}-\lambda_{2}\right)^{2} \rightarrow \infty$ is necessary and sufficient for the formula to hold. It seems feasible to refine our proof technique by splitting the differential equation approximation phase into subphases, possibly to a great enough extent so as to relax our assumption on separation from criticality to the best possible ; however, in the interest of greater clarity, we do not explore such improvements in the present paper. A more detailed discussion of this as well as of what happens when the condition $N\left(\lambda_{1}-\lambda_{2}\right)^{2} \rightarrow \infty$ is not satisfied is included in Subsection 7.1.

Throughout our proofs, we treat $X_{N}(t)$ and $x(t)$ as column vectors.

\section{Preliminaries}

In this section, we discuss the deterministic Lotka-Volterra system. For suitable choices of parameter values, this model becomes the deterministic logistic SIS competition model, and approximates the stochastic logistic SIS competition model over certain timescales.

We further outline the proof of our main result, Theorem 2 .

2.1. A deterministic version of the competition model. The deterministic competitive Lotka-Volterra system represents a community of $k$ mutually competing species described by equations

$$
\frac{d x_{i}(t)}{d t}=x_{i}(t)\left(b_{i}-\sum_{j=1}^{k} a_{i j} x_{j}(t)\right), \quad i=1, \ldots, k,
$$

where $x_{i}(t)$ denotes the population size of the $i$-th species at time $t$. It is assumed that $b_{i}>0$ for all $i$, and $a_{i j}>0$ for all $i, j$. For each $i=1, \ldots, k$, species $i$ would by itself, in the absence of all the other species, exhibit logistic growth, that is, its behaviour would be described by the equation

$$
\frac{d x_{i}(t)}{d t}=x_{i}(t)\left(b_{i}-a_{i i} x_{i}(t)\right), \quad b_{i}, a_{i i}>0
$$


This equation has two fixed points: 0 and $b_{i} / a_{i i}$, the latter being the carrying capacity of species $i$.

The following result of Zeeman (1995) gives simple algebraic criteria on the parameters $b_{i}$ and $a_{i j}$ of (3) which guarantee that, for all strictly positive initial conditions of (3), all but one of the species is driven to extinction, while the one remaining species in the community stabilizes at its own carrying capacity.

We recall that a fixed point $x^{*}$ of a system of ordinary differential equations is globally attractive on a set $U$ if and only if its basin of attraction is equal to $U$. In other words, $x^{*}$ is globally attractive if every solution to the system with initial condition in $U$ converges to $x^{*}$ as $t \rightarrow \infty$.

Theorem 3. (Zeeman 1995) Suppose that system (3) satisfies the inequalities

$$
\begin{aligned}
\frac{b_{j}}{a_{j j}}<\frac{b_{i}}{a_{i j}} \quad \forall i<j \\
\frac{b_{j}}{a_{j j}}>\frac{b_{i}}{a_{i j}} \quad \forall i>j .
\end{aligned}
$$

Then fixed point $\left(\frac{b_{1}}{a_{11}}, 0, \ldots, 0\right)^{T}$ is globally attractive on the interior of $\mathbb{R}_{+}^{k}$.

Clearly, if conditions (4) are satisfied, and $x_{i}(0)=0$ for some $i>1$, then the solution $x(t)$ still converges to $\left(\frac{b_{1}}{a_{11}}, 0, \ldots, 0\right)^{T}$ as $t \rightarrow \infty$, as this case amounts to eliminating species $i$ from the equations.

In the case $\lambda_{1} / \mu_{1}>\lambda_{2} / \mu_{2}>1$, the stochastic competition model can be naturally associated with a particular two-dimensional instance of (3) with $b_{i}=\lambda_{i}-\mu_{i}$ for $i=1,2$, and $a_{i j}=\lambda_{i}$ for $i, j=1,2$, which gives the system (11) with initial conditions $x(0)=\left(x_{1}(0), x_{2}(0)\right)^{T} \in \mathbb{R}_{+}^{2}$. In epidemic modelling, $x_{1}(t)$ and $x_{2}(t)$ represent the proportions of individuals who at time $t$ are infected by strains 1 and 2 respectively, in a closed homogeneously mixing population. By Theorem 3, all solutions with $x_{1}(0)>0$ converge to $\left(\frac{\lambda_{1}-\mu_{1}}{\lambda_{1}}, 0\right)^{T}$ as $t \rightarrow \infty$.

We claim this still holds even when $\lambda_{2} / \mu_{2} \leq 1$. First of all, observe that $x_{2}(t)^{\mu_{1}} / x_{1}(t)^{\mu_{2}}$ is decreasing in $t$, and hence one can see that $d x_{1}(t) / d t \geq 0$ if $x_{1}(t) \leq \varepsilon$ for $\varepsilon>0$ small enough. (We can choose $\varepsilon$ such that $\varepsilon+$ $\left(\varepsilon / x_{1}(0)\right)^{\mu_{2} / \mu_{1}} \leq 1-\mu_{1} / \lambda_{1}$.) Now consider the Lyapunov function

$$
\phi\left(x_{1}, x_{2}\right)=\frac{1}{2}\left(x_{1}+x_{2}-1+\frac{\mu_{1}}{\lambda_{1}}\right)^{2}+x_{2}\left(\frac{\mu_{2}}{\lambda_{2}}-\frac{\mu_{1}}{\lambda_{1}}\right) .
$$


This function is non-negative, and is zero only at the fixed point $\left(x_{1}, x_{2}\right)=$ $\left(1-\frac{\mu_{1}}{\lambda_{1}}, 0\right)$. The derivative is given by

$$
\begin{aligned}
& \frac{d}{d t} \phi\left(x_{1}(t), x_{2}(t)\right) \\
& \quad=\left(x_{1}+x_{2}-1+\frac{\mu_{1}}{\lambda_{1}}\right)\left(\frac{d x_{1}}{d t}+\frac{d x_{2}}{d t}\right)+\left(\frac{\mu_{2}}{\lambda_{2}}-\frac{\mu_{1}}{\lambda_{1}}\right) \frac{d x_{2}}{d t} \\
& \quad=-\lambda_{1} x_{1}\left(x_{1}+x_{2}-1+\frac{\mu_{1}}{\lambda_{1}}\right)^{2}+\left(x_{1}+x_{2}-1+\frac{\mu_{2}}{\lambda_{2}}\right) \frac{d x_{2}}{d t} \\
& \quad=-\lambda_{1} x_{1}\left(x_{1}+x_{2}-1+\frac{\mu_{1}}{\lambda_{1}}\right)^{2}-\lambda_{2} x_{2}\left(x_{1}+x_{2}-1+\frac{\mu_{2}}{\lambda_{2}}\right)^{2},
\end{aligned}
$$

and is non-positive everywhere; furthermore, for any $\varepsilon>0$, it is zero in $\left\{\left(x_{1}, x_{2}\right)^{T}: x_{1} \geq \varepsilon, x_{2} \geq 0, x_{1}+x_{2} \leq 1\right\}$ only at the fixed point $\left(1-\frac{\mu_{1}}{\lambda_{1}}, 0\right)^{T}$. Since $d x_{1}(t) / d t \geq 0$ if $x_{1}(t) \leq \varepsilon$, the set $\left\{\left(x_{1}, x_{2}\right)^{T}: x_{1} \geq \varepsilon, x_{2} \geq 0, x_{1}+x_{2} \leq\right.$ $1\}$ is invariant for the deterministic logistic SIS competition model. As $\varepsilon$ can be taken arbitrarily small, the claim follows.

It is easy to check that each solution $x(t)=\left(x_{1}(t), x_{2}(t)\right)$ to (1) must satisfy

$$
\frac{\left(x_{1}(t)\right)^{\lambda_{2}}}{\left(x_{2}(t)\right)^{\lambda_{1}}}=\frac{\left(x_{1}(0)\right)^{\lambda_{2}}}{\left(x_{2}(0)\right)^{\lambda_{1}}} e^{\left(\mu_{2} \lambda_{1}-\mu_{1} \lambda_{2}\right) t}, \quad \text { for all } t \geq 0 .
$$

Given a solution $(x(t))_{t \geq 0}$ to (1), this relation can be used to calculate the time $t_{a \rightarrow b}$ spent by $(x(t))_{t \geq 0}$ to travel from a point $a=\left(a_{1}, a_{2}\right)^{T}$ to another point $b=\left(b_{1}, b_{2}\right)^{T}$ :

$$
t_{a \rightarrow b}=\frac{\lambda_{2}}{\mu_{2} \lambda_{1}-\mu_{1} \lambda_{2}} \log \left(b_{1} / a_{1}\right)-\frac{\lambda_{1}}{\mu_{2} \lambda_{1}-\mu_{1} \lambda_{2}} \log \left(b_{2} / a_{2}\right) .
$$

The Jacobian of (11) at $\left(\frac{\lambda_{1}-\mu_{1}}{\lambda_{1}}, 0\right)^{T}$ is given by

$$
A=\left(\begin{array}{cc}
-\left(\lambda_{1}-\mu_{1}\right) & -\left(\lambda_{1}-\mu_{1}\right) \\
0 & -\left(\mu_{2}-\lambda_{2} \mu_{1} / \lambda_{1}\right)
\end{array}\right)
$$

and thus has eigenvalues $-\left(\lambda_{1}-\mu_{1}\right)$ and $-\left(\mu_{2}-\lambda_{2} \mu_{1} / \lambda_{1}\right)$, which are real and strictly negative under the assumptions that $\lambda_{1} / \mu_{1}>1$ and $\lambda_{1} / \mu_{1}>\lambda_{2} / \mu_{2}$. By standard theory, the speed of convergence is determined by $-\min \left\{\lambda_{1}-\right.$ $\left.\mu_{1}, \mu_{2}-\lambda_{2} \mu_{1} / \lambda_{1}\right\}$. Indeed, by Chapter VII $\S 29$, Theorem VII in Walter (1998), for any $0<\sigma<\min \left\{\lambda_{1}-\mu_{1}, \mu_{2}-\lambda_{2} \mu_{1} / \lambda_{1}\right\}$, there exist $\eta>0$, $C>0$ such that, if $\left\|\left(x_{1}(0), x_{2}(0)\right)^{T}-\left(\frac{\lambda_{1}-\mu_{1}}{\lambda_{1}}, 0\right)^{T}\right\|_{2}<\eta$, then

$$
\left\|\left(x_{1}(t), x_{2}(t)\right)^{T}-\left(\frac{\lambda_{1}-\mu_{1}}{\lambda_{1}}, 0\right)^{T}\right\|_{2} \leq C e^{-\sigma t} \text { for all } t \geq 0 .
$$

In Subsection 2.2 below, we will give a stronger bound, as well as a lower bound on the speed of convergence. 
EXTINCTION TIME FOR THE WEAKER OF TWO COMPETING SIS EPIDEMICS 11

2.2. Convergence to fixed point. Let $\eta_{1}=\lambda_{1}-\mu_{1}$ and let $\eta_{2}=\mu_{2}-$ $\lambda_{2} \mu_{1} / \lambda_{1}$. Note that $\eta_{1}, \eta_{2}>0$ and $-\eta_{1},-\eta_{2}$ are the eigenvalues of $A$. Let

$$
a=1-\frac{\eta_{2}}{\eta_{1}}
$$

and assume that $a \neq 0$, that is $\eta_{2} \neq \eta_{1}$, When $a=0$, then the matrix (7) has repeated eigenvalues. We will consider this case at the end of this subsection.

We introduce new co-ordinates $\tilde{x}_{1}(t)=x_{1}(t)-\frac{\lambda_{1}-\mu_{1}}{\lambda_{1}}+\frac{1}{a} x_{2}(t)$ and $\tilde{x}_{2}(t)=$ $x_{2}(t)$, and let $\tilde{x}(t)=\left(\tilde{x}_{1}(t), \tilde{x}_{2}(t)\right)^{T}$. In the new co-ordinates, the differential equation (11) is expressed as

$$
\begin{aligned}
\frac{d \tilde{x}_{1}(t)}{d t} & =-\eta_{1} \tilde{x}_{1}(t)-\lambda_{1} \tilde{x}_{1}(t)^{2}-\frac{\eta_{2}\left(\lambda_{1}-\lambda_{2}\right)}{\eta_{1}}\left(\frac{\tilde{x}_{2}(t)}{a}\right)^{2}+\left(\lambda_{1}-\lambda_{2}+\frac{\lambda_{1} \eta_{2}}{\eta_{1}}\right) \tilde{x}_{1}(t) \frac{\tilde{x}_{2}(t)}{a} \\
\frac{d \tilde{x}_{2}(t)}{d t} & =-\eta_{2} \tilde{x}_{2}(t)-\lambda_{2} \tilde{x}_{2}(t) \tilde{x}_{1}(t)+\frac{\lambda_{2}}{a} \frac{\eta_{2}}{\eta_{1}} \tilde{x}_{2}(t)^{2} .
\end{aligned}
$$

Note the diagonal form of the linear terms in the equation, reflecting the fact that $(1,1 / a)$ and $(0,1)$ are the left eigenvectors of the matrix $A$, with eigenvalues $-\eta_{1}$ and $-\eta_{2}$ respectively.

Lemma 1. Suppose that $a \neq 0$. Let $L=\min \left\{\eta_{1}, \eta_{2}\right\}$. Also, let $L_{1}=$ $\left(\lambda_{1}+\left|\lambda_{1}-\lambda_{2}\right|\right) \frac{\eta_{1}+\eta_{2}}{\eta_{1}}$. Suppose $\tilde{x}(0)$ is such that

$$
y(0)=\max \left\{\left|\tilde{x}_{1}(0)\right|, \tilde{x}_{2}(0) /|a|\right\} \leq L / 2 L_{1} .
$$

Then, for all $t \geq 0,\left|\tilde{x}_{1}(t)\right| \leq 2 y(0) e^{-t L}$, and $\tilde{x}_{2}(t) \leq 2|a| y(0) e^{-t L}$.

Proof. We can write, as is standard,

$$
\tilde{x}(t)=\tilde{x}(0)+\int_{0}^{t} F(\tilde{x}(s)) d s,
$$

where $F: \mathbb{R}^{2} \rightarrow \mathbb{R}^{2}$ is given by

$$
F(x)=\left(\begin{array}{c}
F_{1}(x) \\
F_{2}(x)
\end{array}\right)=\left(\begin{array}{c}
-\eta_{1} x_{1}-\lambda_{1} x_{1}^{2}-\frac{\eta_{2}\left(\lambda_{1}-\lambda_{2}\right)}{\eta_{1}}\left(\frac{x_{2}}{a}\right)^{2}+\left(\lambda_{1}-\lambda_{2}+\frac{\lambda_{1} \eta_{2}}{\eta_{1}}\right) x_{1} \frac{x_{2}}{a} \\
-\eta_{2} x_{2}-\lambda_{2} x_{2} x_{1}+\frac{\lambda_{2}}{a} \frac{\eta_{2}}{\eta_{1}} x_{2}^{2}
\end{array}\right) .
$$

We then decompose

$$
F(x)=\tilde{A}\left(\begin{array}{l}
x_{1} \\
x_{2}
\end{array}\right)+\tilde{F}(x),
$$

where

$$
\tilde{A}=\left(\begin{array}{cc}
-\eta_{1} & 0 \\
0 & -\eta_{2},
\end{array}\right)
$$

and

$$
\tilde{F}(x)=\left(\begin{array}{c}
-\lambda_{1} x_{1}^{2}-\frac{\eta_{2}\left(\lambda_{1}-\lambda_{2}\right)}{\eta_{1}}\left(\frac{x_{2}}{a}\right)^{2}+\left(\lambda_{1}-\lambda_{2}+\frac{\lambda_{1} \eta_{2}}{\eta_{1}}\right) x_{1} \frac{x_{2}}{a} \\
-\lambda_{2} x_{2} x_{1}+\frac{\lambda_{2}}{a} \frac{\eta_{2}}{\eta_{1}} x_{2}^{2}
\end{array}\right) .
$$


It is then not hard to check that the solution $\tilde{x}(t)$ satisfies

$$
\tilde{x}(t)=e^{t \tilde{A}} \tilde{x}(0)+\int_{0}^{t} e^{(t-s) \tilde{A}} \tilde{F}(\tilde{x}(s)) d s,
$$

or, equivalently,

$$
\begin{aligned}
& \left(\begin{array}{l}
\tilde{x}_{1}(t) \\
\tilde{x}_{2}(t)
\end{array}\right)=\left(\begin{array}{c}
e^{-t \eta_{1}} \tilde{x}_{1}(0) \\
e^{-t \eta_{2}} x_{2}(0)
\end{array}\right) \\
& +\int_{0}^{t}\left(\begin{array}{c}
e^{-(t-s) \eta_{1}}\left[-\lambda_{1} \tilde{x}_{1}(s)^{2}-\frac{\eta_{2}\left(\lambda_{1}-\lambda_{2}\right)}{\eta_{1}}\left(\frac{\tilde{x}_{2}(s)}{a}\right)^{2}+\left(\lambda_{1}-\lambda_{2}+\frac{\lambda_{1} \eta_{2}}{\eta_{1}}\right) \tilde{x}_{1}(s) \frac{\tilde{x}_{2}(s)}{a}\right] \\
e^{-(t-s) \eta_{2}}\left[-\lambda_{2} \tilde{x}_{2}(s) \tilde{x}_{1}(s)+\frac{\lambda_{2}}{a} \frac{\eta_{2}}{\eta_{1}} \tilde{x}_{2}(s)^{2}\right]
\end{array}\right) d s .
\end{aligned}
$$

Let $y_{1}(t)=\left|\tilde{x}_{1}(t)\right| e^{L t}$, let $y_{2}(t)=\frac{\tilde{x}_{2}(t)}{|a|} e^{L t}$ and let $y(t)=\max \left\{y_{1}(t), y_{2}(t)\right\}$.

Then, from the above,

$$
\begin{aligned}
y_{1}(t) \leq & y_{1}(0)+\int_{0}^{t} e^{L s}\left[\lambda_{1} \tilde{x}_{1}(s)^{2}+\frac{\eta_{2}\left|\lambda_{1}-\lambda_{2}\right|}{\eta_{1}}\left(\frac{\tilde{x}_{2}(s)}{a}\right)^{2}\right. \\
& \left.+\left|\lambda_{1}-\lambda_{2}+\frac{\lambda_{1} \eta_{2}}{\eta_{1}}\right|\left|\tilde{x}_{1}(s)\right| \frac{\tilde{x}_{2}(s)}{|a|}\right] d s \\
\leq & y_{1}(0)+\left(\lambda_{1}+\frac{\eta_{2}\left|\lambda_{1}-\lambda_{2}\right|}{\eta_{1}}+\left|\lambda_{1}-\lambda_{2}\right|+\frac{\lambda_{1} \eta_{2}}{\eta_{1}}\right) \int_{0}^{t} y(s)^{2} e^{-L s} d s \\
\leq & y_{1}(0)+\frac{\eta_{1}+\eta_{2}}{\eta_{1}}\left(\lambda_{1}+\left|\lambda_{1}-\lambda_{2}\right|\right) \int_{0}^{t} y(s)^{2} e^{-L s} d s,
\end{aligned}
$$

and also

$$
y_{2}(t) \leq y_{2}(0)+\lambda_{2} \frac{\eta_{1}+\eta_{2}}{\eta_{1}} \int_{0}^{t} y(s)^{2} e^{-L s} d s
$$

so that

$$
y(t) \leq y(0)+L_{1} \int_{0}^{t} y(s)^{2} e^{-L s} d s .
$$

Now, the equation

$$
z(t)=z(0)+L_{1} \int_{0}^{t} z(s)^{2} e^{-L s} d s
$$

is solved by

$$
z(t)=\frac{L z(0)}{L+L_{1} z(0)\left(e^{-L t}-1\right)},
$$

for all $t$, as long as $z(0)<L / L_{1}$, and, if $z(0) \leq L / 2 L_{1}$, then $z(t) \leq 2 z(0)$ for all $t$. Now a standard argument, considering the difference $z(t)-y(t)$, shows that $y(t) \leq z(t) \leq 2 z(0)$ provided that $y(0) \leq z(0) \leq L / 2 L_{1}$.

So $\left|\tilde{x}_{1}(t)\right| \leq 2 \max \left\{\tilde{x}_{1}(0), \tilde{x}_{2}(0) /|a|\right\} e^{-L t}$ and $\tilde{x}_{2}(t) \leq 2|a| \max \left\{\tilde{x}_{1}(0), \tilde{x}_{2}(0) /|a|\right\} e^{-L t}$ for all $t$, as required. 
EXTINCTION TIME FOR THE WEAKER OF TWO COMPETING SIS EPIDEMICS 13

Lemma 2. Suppose that $a \neq 0$. Let $L, L_{1}$ be as in Lemma 1 . Suppose $\tilde{x}(0)$ is such that

$$
y(0)=\max \left\{\left|\tilde{x}_{1}(0)\right|, \tilde{x}_{2}(0) /|a|\right\} \leq L / 8 L_{1} .
$$

Then, for all $t \geq 0, x_{2}(t) \leq 2 x_{2}(0) e^{-t \eta_{2}}$, and $x_{2}(t) \geq \frac{1}{2} x_{2}(0) e^{-t \eta_{2}}$.

Proof. Defining $y(t)$ as in the proof of Lemma 1 .

$$
\begin{aligned}
x_{2}(t) & \leq x_{2}(0) e^{-t \eta_{2}}+\lambda_{2} \int_{0}^{t} e^{-(t-s) \eta_{2}}\left|\tilde{x}_{1}(s)\right| x_{2}(s) d s+\frac{\lambda_{2}}{|a|} \frac{\eta_{2}}{\eta_{1}} \int_{0}^{t} e^{-(t-s) \eta_{2}} \tilde{x}_{2}(s)^{2} d s \\
& \leq x_{2}(0) e^{-t \eta_{2}}+2 \lambda_{2} \frac{\eta_{1}+\eta_{2}}{\eta_{1}} e^{-t \eta_{2}} y(0) \int_{0}^{t} x_{2}(s) e^{s \eta_{2}} e^{-s L} d s .
\end{aligned}
$$

Letting $\tilde{y}_{2}(t)=x_{2}(t) e^{t \eta_{2}}$, and using the fact that $\lambda_{2} \leq \lambda_{1}+\left|\lambda_{1}-\lambda_{2}\right|$,

$$
\tilde{y}_{2}(t) \leq \tilde{y}_{2}(0)+2 L_{1} y(0) \int_{0}^{t} \tilde{y}_{2}(s) e^{-s L} d s
$$

so, by Gronwall's lemma,

$$
\tilde{y}_{2}(t) \leq \tilde{y}_{2}(0) \exp \left(2 L_{1} y(0) \int_{0}^{t} e^{-s L} d s\right) \leq \tilde{y}_{2}(0) \exp \left(2 L_{1} y(0) / L\right)
$$

so $y_{2}(t) \leq 2 y_{2}(0)$, for all $t$, as required, since $y(0) \leq L / 8 L_{1}$.

Furthermore, for all $t$,

$$
\begin{aligned}
x_{2}(t) & \geq x_{2}(0) e^{-t \eta_{2}}-\lambda_{2} \int_{0}^{t} e^{-(t-s) \eta_{2}}\left|\tilde{x}_{1}(s)\right| x_{2}(s) d s-\frac{\lambda_{2}}{|a|} \frac{\eta_{2}}{\eta_{1}} \int_{0}^{t} e^{-(t-s) \eta_{2}} \tilde{x}_{2}(s)^{2} d s \\
& \geq x_{2}(0) e^{-t \eta_{2}}-2 L_{1} e^{-t \eta_{2}} y(0) \int_{0}^{t} x_{2}(s) e^{s \eta_{2}} e^{-L s} d s \\
& \geq x_{2}(0) e^{-t \eta_{2}}-4 L_{1} y(0) x_{2}(0) e^{-t \eta_{2}} \int_{0}^{t} e^{-s L} d s \\
& \geq x_{2}(0) e^{-t \eta_{2}}-\frac{4 L_{1} y(0)}{L} x_{2}(0) e^{-t \eta_{2}} \\
& \geq \frac{1}{2} x_{2}(0) e^{-t \eta_{2}} .
\end{aligned}
$$

In the case $\eta_{1}=\eta_{2}$, we work with the original variables $x_{1}(t), x_{2}(t)$, and write

$$
\begin{aligned}
& \left(\begin{array}{c}
x_{1}(t)-\frac{\lambda_{1}-\mu_{1}}{\lambda_{1}} \\
x_{2}(t)
\end{array}\right)=e^{t A}\left(\begin{array}{c}
x_{1}(0)-\frac{\lambda_{1}-\mu_{1}}{\lambda_{1}} \\
x_{2}(0)
\end{array}\right)+\int_{0}^{t} e^{A(t-s)} \tilde{F}(x(s)) d s \\
& =e^{t A}\left(\begin{array}{c}
x_{1}(0)-\frac{\lambda_{1}-\mu_{1}}{\lambda_{1}} \\
x_{2}(0)
\end{array}\right) \\
& \quad+\int_{0}^{t} e^{A(t-s)}\left(\begin{array}{c}
-\lambda_{1}\left(x_{1}(s)-\frac{\lambda_{1}-\mu_{1}}{\lambda_{1}}\right)^{2}-\lambda_{1}\left(x_{1}(s)-\frac{\lambda_{1}-\mu_{1}}{\lambda_{1}}\right) x_{2}(s) \\
-\lambda_{2}\left(x_{1}(s)-\frac{\lambda_{1}-\mu_{1}}{\lambda_{1}}\right) x_{2}(s)-\lambda_{2}\left(x_{2}(s)\right)^{2}
\end{array}\right) d s
\end{aligned}
$$


where

$$
e^{t A}=\left(\begin{array}{cc}
e^{-t\left(\lambda_{1}-\mu_{1}\right)} & -\left(\lambda_{1}-\mu_{1}\right) t e^{-t\left(\lambda_{1}-\mu_{1}\right)} \\
0 & e^{-t\left(\lambda_{1}-\mu_{1}\right)}
\end{array}\right) .
$$

Let $y(t)=e^{t\left(\lambda_{1}-\mu_{1}\right) / 2} \max \left\{\left|x_{1}(t)-\left(\lambda_{1}-\mu_{1}\right) / \lambda_{1}\right|, x_{2}(t)\right\}$.

Lemma 3. Suppose that $a=0$. Assume that $y(0) \leq\left(\lambda_{1}-\mu_{1}\right) / 32\left(\lambda_{1}+\lambda_{2}\right)$. Then, for all $t \geq 0,\left|x_{1}(t)-\left(\lambda_{1}-\mu_{1}\right) / \lambda_{1}\right| \leq 4 y(0) e^{-t\left(\lambda_{1}-\mu_{1}\right) / 2}$ and $x_{2}(t) \leq$ $4 y(0) e^{-t\left(\lambda_{1}-\mu_{1}\right) / 2}$.

Proof. Since $\lambda_{2}\left[\left(\lambda_{1}-\mu_{1}\right) t+\lambda_{1} / \lambda_{2}\right] e^{-t\left(\lambda_{1}-\mu_{1}\right) / 2} \leq 2\left(\lambda_{1}+\lambda_{2}\right)$ and $\left[\left(\lambda_{1}-\mu_{1}\right) t+\right.$ 1] $e^{-t\left(\lambda_{1}-\mu_{1}\right) / 2} \leq 2$, we have

$$
\begin{aligned}
& \left|x_{1}(t)-\left(\lambda_{1}-\mu_{1}\right) / \lambda_{1}\right| \\
& \quad \leq 2 y(0) e^{-t\left(\lambda_{1}-\mu_{1}\right) / 2}+4\left(\lambda_{1}+\lambda_{2}\right) e^{-t\left(\lambda_{1}-\mu_{1}\right) / 2} \int_{0}^{t} y(s)^{2} e^{-s\left(\lambda_{1}-1\right) / 2} d s,
\end{aligned}
$$

and

$$
x_{2}(t) \leq y(0) e^{-t\left(\lambda_{1}-\mu_{1}\right) / 2}+2 \lambda_{2} e^{-t\left(\lambda_{1}-\mu_{1}\right) / 2} \int_{0}^{t} y(s)^{2} e^{-s\left(\lambda_{1}-\mu_{1}\right) / 2} d s .
$$

It follows using the same argument as in the proof of Lemma 1 that

SO

$$
y(t) \leq 2 y(0)+4\left(\lambda_{1}+\lambda_{2}\right) \int_{0}^{t} y(s)^{2} e^{-s\left(\lambda_{1}-\mu_{1}\right) / 2} d s,
$$

$$
y(t) \leq \frac{\left(\lambda_{1}-\mu_{1}\right) y(0)}{\left(\lambda_{1}-\mu_{1}\right) / 2+8\left(\lambda_{1}+\lambda_{2}\right) y(0)\left(e^{-t\left(\lambda_{1}-\mu_{1}\right) / 2}-1\right)} .
$$

Thus if $y(0) \leq\left(\lambda_{1}-\mu_{1}\right) / 32\left(\lambda_{1}+\lambda_{2}\right), y(t) \leq 4 y(0)$, so $\left|x_{1}(t)-\left(\lambda_{1}-\mu_{1}\right) / \lambda_{1}\right| \leq$ $4 \max \left\{x_{1}(0), x_{2}(0)\right\} e^{-t\left(\lambda_{1}-\mu_{1}\right) / 2}$ and $x_{2}(t) \leq 4 \max \left\{x_{1}(0), x_{2}(0)\right\} e^{-t\left(\lambda_{1}-\mu_{1}\right) / 2}$.

Lemma 4. Suppose that $a=0$. Assume that $y(0) \leq\left(\lambda_{1}-\mu_{1}\right) / 32\left(\lambda_{1}+\lambda_{2}\right)$. Then, for all $t \geq 0, x_{2}(t) \leq 2 x_{2}(0) e^{-t\left(\lambda_{1}-\mu_{1}\right)}$ and $x_{2}(t) \geq \frac{1}{2} x_{2}(0) e^{-t\left(\lambda_{1}-\mu_{1}\right)}$.

Proof. Letting $\tilde{y}_{2}(t)=x_{2}(t) e^{\left(\lambda_{1}-\mu_{1}\right) t}$, we have, using Lemma 3 ,

so

$$
\tilde{y}_{2}(t) \leq \tilde{y}_{2}(0)+4 \lambda_{2} y(0) \int_{0}^{t} \tilde{y}_{2}(s) e^{-s\left(\lambda_{1}-\mu_{1}\right) / 2} d s,
$$

$\tilde{y}_{2}(t) \leq \tilde{y}_{2}(0) \exp \left(8 \lambda_{2} y(0) /\left(\lambda_{1}-\mu_{1}\right)\right)$, so $x_{2}(t) \leq 2 x_{2}(0) e^{-t\left(\lambda_{1}-\mu_{1}\right)}$, since $y(0) \leq\left(\lambda_{1}-\mu_{1}\right) / 32 \lambda_{2}$. Finally,

$$
\begin{aligned}
\tilde{y}_{2}(t) & \geq \tilde{y}_{2}(0)-4 \lambda_{2} y(0) \int_{0}^{t} \tilde{y}_{2}(s) e^{-s\left(\lambda_{1}-\mu_{1}\right) / 2} d s \\
& \geq \tilde{y}_{2}(0)-8 \lambda_{2} y(0) x_{2}(0) \int_{0}^{t} e^{-s\left(\lambda_{1}-\mu_{1}\right) / 2} d s \\
& \geq x_{2}(0)-16 \lambda_{2} y(0) x_{2}(0) /\left(\lambda_{1}-\mu_{1}\right),
\end{aligned}
$$

so $y_{2}(t) \geq \frac{1}{2} x_{2}(0) e^{-t\left(\lambda_{1}-\mu_{1}\right)}$. 
2.3. Proof strategy. As we mentioned in the introduction, we break up the analysis of the competition process into phases as follows.

Initial phase ('burn-in' period): By standard theory, see for instance Kurtz (1970) or Darling and Norris (2008), over a fixed length (i.e. independent of $N$ ) interval $\left[0, t_{0}\right], X_{N}(t) / N$, is well approximated by the solution $x(t)$ of (11) starting from the same (or nearby) initial condition. We will choose $t_{0}$ such that $x_{1}\left(t_{0}\right)$ is close to $\frac{\lambda_{1}-\mu_{1}}{\lambda_{1}}$ and $x_{2}\left(t_{0}\right)$ is very small. (This is possible by Theorem 3 and the discussion following it, since the fixed point $\left(\left(\lambda_{1}-\mu_{1}\right) / \lambda_{1}, 0\right)^{T}$ is stable. $)$

Intermediate phase: After time $t_{0}$, we linearise (1) and its stochastic analogue around $\left(\left(\lambda_{1}-\mu_{1}\right) / \lambda_{1}, 0\right)^{T}$, and use this to show that $x_{N}(t)=$ $X_{N}(t) / N$ follows the solution $x(t)$ to (11) for quite a long time after $t_{0}$. Our approach here is a variation on standard martingale techniques adapted to exploit the proximity of a stable fixed point.

We choose the time $t_{N, 1}$ as the time when $x_{2}(t)$ drops down to $N^{-1 / 4}$ (so $X_{N, 2}(t)$ will be around $\left.N^{3 / 4}\right)$.

Final phase: This phase starts with $X_{N, 1}(t)$ near $\frac{\lambda_{1}-\mu_{1}}{\lambda_{1}} N$ and $X_{N, 2}(t)$ near $N^{3 / 4}$. From then onwards, 'logistic effects' can be ignored, and the path of $X_{N, 2}(t)$ can be sandwiched between the paths of two subcritical linear birth and death processes also starting near $N^{3 / 4}$. Since the time to extinction of a linear birth and death process is well known, we obtain the distribution of the remaining time until the extinction of $X_{N, 2}(t)$.

Theorem 2 follows by adding up the times spent in each phase.

\section{INITIAL PHASE}

Lemma 5. Let $x_{N}(t)=X_{N}(t) / N$. Let $t_{0}>0$, let $0<\delta \leq(\log 4) t_{0}\left(\lambda_{1}+1\right)$, and assume that $\left\|x_{N}(0)-x(0)\right\|_{1} \leq \delta$. Then

$$
\mathbb{P}\left(\sup _{t \leq t_{0}}\left\|x_{N}(t)-x(t)\right\|_{1} \leq 2 \delta e^{\left(5 \lambda_{1}+1\right) t_{0}}\right) \leq 4 e^{-\delta^{2} N / 4 t_{0}\left(\lambda_{1}+1\right)} .
$$

Proof. The general method is described in, for instance, Darling and Norris (2008). In the next section, we will develop a variant adapted to the case where the solution $x(t)$ is in the neighbourhood of a stable fixed point.

As is standard, we write

$$
x(t)=x(0)+\int_{0}^{t} F(x(s)) d s,
$$

where $F: \mathbb{R}^{2} \rightarrow \mathbb{R}^{2}$ is given by

$$
F(x)=\left(\begin{array}{l}
F_{1}(x) \\
F_{2}(x)
\end{array}\right)=\left(\begin{array}{l}
\lambda_{1} x_{1}\left(1-x_{1}-x_{2}\right)-x_{1} \\
\lambda_{2} x_{2}\left(1-x_{1}-x_{2}\right)-x_{2}
\end{array}\right) .
$$

Also, $x_{N}(t)=X_{N}(t) / N$ satisfies

$$
x_{N}(t)=x_{N}(0)+\int_{0}^{t} F\left(x_{N}(s)\right) d s+M_{N}(t),
$$


where $\left(M_{N}(t)\right)$ is a zero-mean martingale.

We can take $5 \lambda_{1}+1$ for a Lipschitz constant of $F$ with respect to $\|\cdot\|_{1}$ in the subset of $\mathbb{R}^{2}$ given by $\left\{x=\left(x_{1}, x_{2}\right)^{T}: 0 \leq x_{1}, x_{2} \leq 1\right\}$. Then for $t \leq t_{0}$,

$$
\begin{aligned}
& \left\|x_{N}(t)-x(t)\right\|_{1} \\
& \quad \leq\left\|x_{N}(0)-x(0)\right\|_{1}+\int_{0}^{t_{0}}\left\|F\left(x_{N}(s)\right)-F(x(s))\right\|_{1} d s+\sup _{t \leq t_{0}}\left\|M_{N}(t)\right\|_{1} \\
& \quad \leq\left\|x_{N}(0)-x(0)\right\|_{1}+\left(5 \lambda_{1}+1\right) \int_{0}^{t_{0}} \sup _{u \leq s}\left\|x_{N}(u)-x(u)\right\|_{1} d s+\sup _{t \leq t_{0}}\left\|M_{N}(t)\right\|_{1} \\
& \quad \leq\left(\left\|x_{N}(0)-x(0)\right\|_{1}+\sup _{t \leq t_{0}}\left\|M_{N}(t)\right\|_{1}\right) e^{\left(5 \lambda_{1}+1\right) t_{0}},
\end{aligned}
$$

so

$$
\sup _{t \leq t_{0}}\left\|x_{N}(t)-x(t)\right\|_{1} \leq\left(\left\|x_{N}(0)-x(0)\right\|_{1}+\sup _{t \leq t_{0}}\left\|M_{N}(t)\right\|_{1}\right) e^{\left(5 \lambda_{1}+1\right) t_{0}} .
$$

For $\theta \in \mathbb{R}^{2}$, let

$$
\begin{aligned}
& Z_{N}(t, \theta) \\
& =\exp \left(\theta^{T}\left(x_{N}(t)-x_{N}(0)\right)-\int_{0}^{t} d s \sum_{y} q_{N}\left(x_{N}(s), x_{N}(s)+y\right)\left(e^{\theta^{T} y}-1\right)\right) \\
& =\exp \left(\theta^{T} M_{N}(t)-\int_{0}^{t} d s \sum_{y} q_{N}\left(x_{N}(s), x_{N}(s)+y\right)\left(e^{\theta^{T} y}-1-\theta^{T} y\right)\right),
\end{aligned}
$$

where $q_{N}(x, x+y)$ denotes the rate of $y$ jumps of $x_{N}(t)$ when in state $x$. Then $\left(Z_{N}(t, \theta)\right)$ is a mean 1 martingale. Note that, for our model, the jumps $y$ are of the form $( \pm 1 / N, 0)^{T}$ and $(0, \pm 1 / N)^{T}$, and $F(x)=\sum_{y} q_{N}(x, x+y) y$.

Using the identity $e^{z}-1-z=z^{2} \int_{r=0}^{1} e^{r z}(1-r) d r$, we write $Z_{N}(t, \theta)$ as $Z_{N}(t, \theta)$

$$
=\exp \left(\theta^{T} M_{N}(t)-\int_{0}^{t} \sum_{y} q_{N}\left(x_{N}(s), x_{N}(s)+y\right)\left(\theta^{T} y\right)^{2}\left(\int_{0}^{1} e^{r \theta^{T} y}(1-r) d r\right) d s\right) .
$$

As the jumps $y$ are of the form $( \pm 1 / N, 0)^{T}$ and $(0, \pm 1 / N)^{T}$,

$$
\int_{0}^{1} e^{r \theta^{T} y}(1-r) d r \leq \frac{1}{2} e^{\gamma}
$$

for $\|\theta\| \leq \gamma N$. It follows that, for all $t$,

$$
Z_{N}(t, \theta) \geq \exp \left(\theta^{T} M_{N}(t)-\frac{1}{2} e^{\gamma} \int_{0}^{t} \sum_{y} q_{N}\left(x_{N}(s), x_{N}(s)+y\right)\left(\theta^{T} y\right)^{2} d s\right) .
$$

In particular, let $\theta_{1}, \theta_{2} \in \mathbb{R}$, and let $\theta^{i}=\theta_{i} e_{i}$ (where $e_{i}$ is the unit vector with 1 in the $i$-th co-ordinate). Then, for all $t$, for $i=1,2$,

$$
Z_{N}\left(t, \theta^{i}\right) \geq \exp \left(\theta_{i} M_{N, i}(t)-\frac{1}{2} e^{\gamma} \theta_{i}^{2} t\left(\lambda_{1}+1\right) \frac{1}{N}\right) .
$$


For $\delta>0$, let $T_{+}^{i}(\delta)=\inf \left\{t \geq 0: M_{N, i}(t)>\delta\right\}$ and let $T_{-}^{i}(\delta)=\inf \{t \geq 0:$ $\left.M_{N, i}(t)<-\delta\right\}$. By optional stopping and Markov inequality,

$$
\mathbb{P}\left(T_{+}^{i}(\delta) \leq t_{0}\right) \leq \exp \left(-\theta_{i} \delta+\frac{1}{2} e^{\gamma} \theta_{i}^{2} t_{0}\left(\lambda_{1}+1\right) \frac{1}{N}\right)
$$

Choosing $\gamma=\log 2, \theta_{i}=N \delta / 2 t_{0}\left(\lambda_{1}+1\right) 0 \leq \theta_{i} \leq \gamma N$, as long as $\delta \leq$ $(\log 4) t_{0}\left(\lambda_{1}+1\right)$. We then obtain, for $i=1,2$,

$$
\mathbb{P}\left(T_{+}^{i}(\delta) \leq t_{0}\right) \leq e^{-\delta^{2} N / 4 t_{0}\left(\lambda_{1}+1\right)} .
$$

Arguing similarly about negative $\delta$, we see that, for $i=1,2$,

$$
\mathbb{P}\left(\sup _{t \leq t_{0}}\left|M_{N, i}(t)\right|>\delta\right) \leq 2 e^{-\delta^{2} N / 4 t_{0}\left(\lambda_{1}+1\right)} .
$$

Then the lemma follows from (13).

\section{INTERMEDIATE PHASE: LONG-TERM DIFFERENTIAL EQUATION APPROXIMATION}

As in the previous section, we use $x_{N}(t)$ to denote $X_{N}(t) / N$. The aim of this section is to show that $x_{N}(t)$ stays concentrated around the solution $x(t)$ of the deterministic system (1) for a long period of time, provided $x_{N}(0)$ and $x(0)$ are close to each other, and $x(0)$ is close to the fixed point $\left(\left(\lambda_{1}-\mu_{1}\right) / \lambda_{1}, 0\right)^{T}$.

We will treat in detail only the case where the eigenvalues of matrix $A$ are distinct, so that $a \neq 0$. By analogy with the notation in Section 2.2, we let $\tilde{x}_{N, 1}(t)=x_{N, 1}(t)-\frac{\lambda_{1}-\mu_{1}}{\lambda_{1}}+\frac{1}{a} x_{N, 2}(t), \tilde{x}_{N, 2}(t)=x_{N, 2}(t)$, and we let $\tilde{x}_{N}(t)$ be the column vector with components $\tilde{x}_{N, 1}(t)$ and $\tilde{x}_{N, 2}(t)$.

As in Section 2.2, let $L=\min \left\{\eta_{1}, \eta_{2}\right\}$ and let $L_{1}=\left(\lambda_{1}+\left|\lambda_{1}-\lambda_{2}\right|\right) \frac{\eta_{1}+\eta_{2}}{\eta_{1}}$.

Let $b=\frac{|a|+1}{|a|}$, let $a_{1}=\left(2 \eta_{1}\right)^{-1} b^{2}\left(\lambda_{1}+\mu_{1}+\lambda_{2}+\mu_{2}\right)$ and let $a_{2}=$ $\left(2 \eta_{2}\right)^{-1}\left(\lambda_{2}+\mu_{2}\right)$. Let also $\tilde{L}=\max \left\{\eta_{1}, \eta_{2}\right\}$.

Lemma 6. Let $\omega$ satisfy $0<\omega<4(\log 2)^{2} N a_{i} / b^{2}$ for $i=1,2$. For $t>0$, let

$$
f_{N}(t)=\max \left\{\left|\tilde{x}_{N, 1}(t)-\tilde{x}_{1}(t)\right|,\left|\tilde{x}_{N, 2}(t)-\tilde{x}_{2}(t)\right||a|^{-1}\right\},
$$

and suppose that

$$
f_{N}(0) \leq e^{\tilde{L}}\left(\frac{\omega\left(a_{1}+a_{2}\right)}{N}\right)^{1 / 2} .
$$

Suppose also that $y(0)=\max \left\{\tilde{x}_{1}(0),|a|^{-1} \tilde{x}_{2}(0)\right\} \leq L / 8 L_{1}$. Then

$$
\mathbb{P}\left(\sup _{t \leq\left\lceil e^{\omega / 8}\right\rceil} f_{N}(t)>8 e^{\tilde{L}}\left(\frac{\omega\left(a_{1}+a_{2}\right)}{N}\right)^{1 / 2}\right) \leq 8 e^{-\omega / 8} .
$$

The proof of Lemma 6 will follow shortly. By standard theory,

$$
\tilde{x}_{N}(t)=\tilde{x}_{N}(0)+\int_{0}^{t} F\left(\tilde{x}_{N}(s)\right) d s+M_{N}(t),
$$


where $\left(M_{N}(t)\right)$ is a martingale, and $F(x)$ is the drift of $\left(\tilde{x}_{N}(t)\right)$ when in state $x$, given as in (10). Analogously to the deterministic process $x(t)$,

$$
\begin{aligned}
\tilde{x}_{N}(t) & =e^{\tilde{A} t} \tilde{x}_{N, 0}+\int_{0}^{t} e^{\tilde{A}(t-s)} \tilde{F}\left(X_{N}(s)\right) d s+\int_{0}^{t} e^{\tilde{A}(t-s)} d M_{N}(s) \\
= & \left(\begin{array}{c}
e^{-t \eta_{1}} \tilde{x}_{N, 1}(0) \\
e^{-t \eta_{2}} \tilde{x}_{N, 2}(0)
\end{array}\right) \\
& +\int_{0}^{t}\left(\begin{array}{c}
e^{-(t-s) \eta_{1}}\left[-\lambda_{1} \tilde{x}_{N, 1}(s)^{2}-\frac{\eta_{2}\left(\lambda_{1}-\lambda_{2}\right)}{\eta_{1}}\left(\frac{\tilde{x}_{N, 2}(s)}{a}\right)^{2}+\left(\lambda_{1}-\lambda_{2}+\frac{\lambda_{1} \eta_{2}}{\eta_{1}}\right) \frac{\tilde{x}_{N, 1}(s) \tilde{x}_{N, 2}(s)}{a}\right] \\
e^{-(t-s) \eta_{2}}\left[-\lambda_{2} \tilde{x}_{N, 2}(s) \tilde{x}_{N, 1}(s)+\frac{\lambda_{2} \eta_{2}}{\eta_{1} a} \tilde{x}_{N, 2}(s)^{2}\right]
\end{array}\right) d s \\
& +\quad+\int_{0}^{t} e^{\tilde{A}(t-s)} d M_{N}(s),
\end{aligned}
$$

where $\tilde{A}$ is as in (11) and $\tilde{F}$ is as in (12). (This formula is proven in the same way as the 'variation of constants' formula in Lemma 4.1 in Barbour and Luczak (2012).)

The following analysis of the martingale transform $\int_{0}^{t} e^{(t-s) \tilde{A}} d M_{N}(s)$ is generic, and applicable in the context of any finite dimensional Markov chain.

Lemma 7. Let $(X(t))$ be a Markov chain with state space $S \subseteq \mathbb{R}^{k}$, where $k$ is a positive integer. For $x, y \in \mathbb{R}^{k}$ such that $x, x+y \in S$, let $\tilde{q}(x, x+y)$ denote the rate of jump $y$ from $x$, and assume that $\|y\| \leq B$ for each possible jump $y$. Suppose further that, for each $x \in S$, the drift $F(x):=\sum_{y} \tilde{q}(x, x+y) y$ at $x$ can be written in the form

$$
F(x)=\tilde{A} x+\tilde{F}(x),
$$

where $\tilde{A}$ is a $k \times k$ matrix with non-positive eigenvalues. Let $(M(t))$ be the corresponding Dynkin martingale, that is,

$$
X(t)=X(0)+\int_{0}^{t}(\tilde{A} X(s)+\tilde{F}(X(s))) d s+M(t) .
$$

Given a vector $\mathbf{e} \in \mathbb{R}^{k}$ with $\|\mathbf{e}\|=1$, let $v_{\mathbf{e}}(x, u)=\sum_{y} \tilde{q}(x, x+y)\left(\mathbf{e}^{T} e^{\tilde{A} u} y\right)^{2}$, and, for $K>0$, let $S_{\mathbf{e}}(K)=\inf \left\{t \geq 0: \int_{0}^{t} v_{\mathbf{e}}(X(s), t-s) d s>K\right\}$. Let $S_{i}(K)=S_{\mathbf{e}_{i}}(K)$, where $\mathbf{e}_{i}$ is a unit vector with 1 in the $i$-th co-ordinate.

Suppose $\mathbf{e}$ is a unit eigenvector of $\tilde{A}$ with eigenvalue $-\eta$, where $\eta \geq 0$. Then, given $K, \sigma>0$, and $0<\omega<4(\log 2)^{2} K / B^{2}$

$$
\mathbb{P}\left(\sup _{t \leq \sigma\left\lceil e^{\omega / 8}\right\rceil \wedge S_{\mathbf{e}}(K)}\left|\int_{0}^{t} \mathbf{e}^{T} e^{\tilde{A}(t-s)} d M(s)\right|>e^{\sigma \eta} \sqrt{\omega K}\right) \leq 4 e^{-\omega / 8} .
$$

Given numbers $K_{1}, \ldots, K_{k}>0$, let $S\left(K_{1}, \ldots, K_{k}\right)=\wedge_{i=1}^{k} S_{i}\left(K_{i}\right)$. Let $\omega$ satisfy $0<\omega<4(\log 2)^{2} K_{i} / B^{2}$ for each $i$. Then, for an arbitrary unit 
EXTINCTION TIME FOR THE WEAKER OF TWO COMPETING SIS EPIDEMICS 19

vector $\mathbf{e}$,

$$
\begin{array}{r}
\mathbb{P}\left(\sup _{t \leq \sigma\left\lceil e^{\omega / 8}\right\rceil \wedge S\left(K_{1}, \ldots, K_{k}\right)}\left|\int_{0}^{t} \mathbf{e}^{T} e^{\tilde{A}(t-s)} d M(s)\right|>e^{\sqrt{k} \sigma \min \left\{\|\tilde{A}\|_{1},\|\tilde{A}\|_{\infty}\right.} \sqrt{\omega} \sqrt{\sum_{i} K_{i}}\right) \\
\leq 4 k e^{-\omega / 8} .
\end{array}
$$

Proof. Fix a time $\tau>0$, and consider $M^{\tau}$ given by

$$
M^{\tau}(t)=\int_{0}^{t \wedge \tau} e^{\tilde{A}(\tau-s)} d M(s)=\int_{0}^{t \wedge \tau} e^{\tilde{A}(\tau-s)}\left(d X(s)-\sum_{y} y \tilde{q}(X(s), X(s)+y) d s\right) .
$$

Then $\left(M^{\tau}(t)\right)$ is a zero mean martingale. Also, for each $t \geq 0, \int_{0}^{t} e^{\tilde{A}(t-s)} d M(s)=$ $M^{t}(t)$. We now define

$Y^{\tau}(t)$

$$
\begin{aligned}
& =\int_{0}^{t \wedge \tau} e^{\tilde{A}(\tau-s)} F s(X(s)) d s+M^{\tau}(t)=\int_{0}^{t \wedge \tau} \sum_{y} e^{\tilde{A}(\tau-s)} y \tilde{q}(X(s), X(s)+y) d s \\
& +\int_{0}^{t \wedge \tau} e^{\tilde{A}(\tau-s)}\left(d X(s)-\sum_{y} y \tilde{q}(X(s), X(s)+y) d s\right)=\int_{0}^{t \wedge \tau} e^{\tilde{A}(\tau-s)} d X(s) .
\end{aligned}
$$

For $\theta \in \mathbb{R}^{k}$, let $R^{\tau}(t, \theta)$ be defined by

$$
R^{\tau}(t, \theta)=e^{\theta^{T} Y^{\tau}(t)}-\int_{0}^{t \wedge \tau} \sum_{y} \tilde{q}(X(r), X(r)+y)\left(e^{\theta^{T}\left[Y^{\tau}(r)+e^{\tilde{A}(\tau-r)} y\right]}-e^{\theta^{T} Y^{\tau}(r)}\right) d r
$$

Then $\left(R^{\tau}(t, \theta)\right)$ is a martingale. Also, for $\theta \in \mathbb{R}^{k},\left(Z^{\tau}(t, \theta)\right)$ given by

$$
Z^{\tau}(t, \theta)=e^{\theta^{T} Y^{\tau}(t)} \exp \left(-\int_{0}^{t \wedge \tau} \sum_{y} \tilde{q}(X(s), X(s)+y)\left(e^{\theta^{T} e^{\tilde{A}(\tau-s)} y}-1\right) d s\right)
$$


is a mean 1 martingale, since, for all $t$, using integration by parts,

$$
\begin{aligned}
Z^{\tau}(t, \theta) & =e^{\theta^{T} Y^{\tau}(0)} \\
& +\int_{0}^{t} \exp \left(-\int_{0}^{r \wedge \tau} \sum_{y} \tilde{q}(X(s), X(s)+y)\left(e^{\theta^{T} e^{\tilde{A}(\tau-s)} y}-1\right) d s\right) d e^{\theta^{T} Y^{\tau}(r)} \\
& -\int_{0}^{t \wedge \tau} e^{\theta^{T} Y^{\tau}(r)} \sum_{y} \tilde{q}(X(r), X(r)+y)\left(e^{\theta^{T} e^{\tilde{A}(\tau-r)} y}-1\right) \\
& \times \exp \left(-\int_{0}^{r \wedge \tau} \sum_{y} \tilde{q}(X(s), X(s)+y)\left(e^{\theta^{T} e^{\tilde{A}(\tau-s)} y}-1\right) d s\right) d r \\
= & +\int_{0}^{t \wedge \tau} \exp \left(-\int_{0}^{r \wedge \tau} \sum_{y} \tilde{q}(X(s), X(s)+y)\left(e^{\theta^{T} e^{\tilde{A}(\tau-s)} y}-1\right) d s\right) \\
& \times\left(d e^{\theta^{T} Y^{\tau}(r)}-\sum_{y} \tilde{q}(X(r), X(r)+y)\left(e^{\theta^{T}\left[Y^{\tau}(r)+e^{\tilde{A}(\tau-r)} y\right]}-e^{\theta^{T} Y^{\tau}(r)}\right) d r\right) \\
= & +\int_{0}^{t \wedge \tau} \exp \left(-\int_{0}^{r \wedge \tau} \sum_{y} \tilde{q}(X(s), X(s)+y)\left(e^{\theta^{T} e^{\tilde{A}(\tau-s)} y}-1\right) d s\right) d R^{\tau}(r, \theta) .
\end{aligned}
$$

Note that

$$
\begin{aligned}
& Z^{\tau}(t, \theta) \\
& \quad=\exp \left(\theta^{T} Y^{\tau}(t)-\int_{0}^{t \wedge \tau} \sum_{y} \tilde{q}(X(s), X(s)+y) \theta^{T} e^{\tilde{A}(\tau-s)} y d s\right) \\
& \quad \times \exp \left(-\int_{0}^{t \wedge \tau} \sum_{y} \tilde{q}(X(s), X(s)+y)\left(e^{\theta^{T} e^{\tilde{A}(\tau-s)} y}-1-\theta^{T} e^{\tilde{A}(\tau-s)} y\right) d s\right) \\
& \quad=\exp \left(\theta^{T} M^{\tau}(t)-\int_{0}^{t \wedge \tau} \sum_{y} \tilde{q}(X(s), X(s)+y)\left(e^{\theta^{T} e^{\tilde{A}(\tau-s)} y}-1-\theta^{T} e^{\tilde{A}(\tau-s)} y\right) d s\right) .
\end{aligned}
$$

Using the identity $e^{z}-1-z=z^{2} \int_{r=0}^{1} e^{r z}(1-r) d r$, we can rewrite that as

$$
\begin{aligned}
& Z^{\tau}(t, \theta)=\exp \left(\theta^{T} M^{\tau}(t)\right. \\
& \left.\quad-\int_{0}^{t \wedge \tau} \sum_{y} \tilde{q}(X(s), X(s)+y)\left(\theta^{T} e^{\tilde{A}(\tau-s)} y\right)^{2}\left(\int_{0}^{1} e^{r \theta^{T} e^{\tilde{A}(\tau-s)} y}(1-r) d r\right) d s\right) .
\end{aligned}
$$

We have assumed that each jump $y$ satisfies $\|y\| \leq B$, so, since $\tilde{A}$ has negative eigenvalues, $\left\|e^{\tilde{A}(\tau-s)} y\right\|$ is also always bounded by $B$. Hence, for $\|\theta\| \leq \Gamma$,

$$
\int_{0}^{1} e^{r \theta^{T} e^{\tilde{A}(\tau-s)} y}(1-r) d r \leq \frac{1}{2} e^{B \Gamma}
$$


EXTINCTION TIME FOR THE WEAKER OF TWO COMPETING SIS EPIDEMICS 21

It follows that, for all $t$,

$Z^{\tau}(t, \theta) \geq \exp \left(\theta^{T} M^{\tau}(t)-\frac{1}{2} e^{B \Gamma} \int_{0}^{t \wedge \tau} \sum_{y} \tilde{q}(X(s), X(s)+y)\left(\theta^{T} e^{\tilde{A}(\tau-s)} y\right)^{2} d s\right)$.

Writing $\theta=\|\theta\| \mathbf{e}$, for a unit vector $\mathbf{e} \in \mathbb{R}^{k}$,

$$
Z^{\tau}(t, \theta) \geq \exp \left(\|\theta\| \mathbf{e}^{T} M^{\tau}(t)-\frac{1}{2} e^{B \Gamma}\|\theta\|^{2} \int_{0}^{t \wedge \tau} v_{\mathbf{e}}(X(s), \tau-s) d s\right) .
$$

Recall that for $K>0, S_{\mathbf{e}}(K)=\inf \left\{t \geq 0: \int_{0}^{t} v_{\mathbf{e}}(X(s), t-s) d s>K\right\}$. Given any path of the process $(X(t)), \int_{0}^{t} v_{\mathbf{e}}(X(s), t-s) d s$ is continuous in $t$, and so $S_{\mathbf{e}}(K)$ is a stopping time. Also, for $\tau>0, K>0$, let

$$
S_{\mathbf{e}}^{\tau}(K)=\inf \left\{t \geq 0: \int_{0}^{t \wedge \tau} v_{\mathbf{e}}(X(s), \tau-s) d s>K\right\} .
$$

Then necessarily either $S_{\mathbf{e}}^{\tau}(K) \leq \tau$ or $S_{\mathbf{e}}^{\tau}(K)=\infty$; and $S_{\mathbf{e}}^{\tau}(K) \leq \tau$ if and only if $S_{\mathbf{e}}(K) \leq \tau$. Therefore, also, if $S_{\mathbf{e}}(K)>\tau$, then $S_{\mathbf{e}}^{t}(K)>t$ for all $t \leq \tau$.

For $t, \tau>0$, unit vector e, let $M_{\mathbf{e}}^{\tau}(t)=e^{T} M^{\tau}(t)$. Also given $\delta>0$, let $T_{\mathbf{e}}^{\tau,+}(\delta)=\inf \left\{t \geq 0: M_{\mathbf{e}}^{\tau}(t)>\delta\right\}$, and let $T_{i}^{\tau,-}(\delta)=\inf \left\{t \geq 0: M_{\mathbf{e}}^{\tau}(t)<\right.$ $-\delta\}$. Then $T_{\mathbf{e}}^{\tau, \pm}(\delta) \leq \tau$ or $T_{\mathbf{e}}^{\tau, \pm}(\delta)=\infty$.

Given $K>0$, on the event $\left\{T_{\mathbf{e}}^{\tau,+}(\delta) \leq \tau \wedge S_{\mathbf{e}}^{\tau}(K)\right\}$,

$$
Z^{\tau}\left(T_{\mathbf{e}}^{\tau,+}(\delta),\|\theta\| \mathbf{e}\right) \geq \exp \left(\|\theta\| \delta-\frac{1}{2} e^{B \Gamma}\|\theta\|^{2} K\right) .
$$

By optional stopping and Markov inequality,

$$
\mathbb{P}\left(T_{\mathbf{e}}^{\tau,+}(\delta) \leq \tau \wedge S_{\mathbf{e}}^{\tau}(K)\right) \leq \exp \left(-\|\theta\| \delta+\frac{1}{2} e^{B \Gamma}\|\theta\|^{2} K\right)
$$

Choosing $\|\theta\|=\delta e^{-B \Gamma} / K$, and assuming $\delta \leq \Gamma K e^{B \Gamma}$ so that $\|\theta\| \leq \Gamma$, we obtain

and, similarly,

$$
\mathbb{P}\left(T_{\mathbf{e}}^{\tau,+}(\delta) \leq \tau \wedge S_{\mathbf{e}}^{\tau}(K)\right) \leq e^{-\delta^{2} / 2 K e^{B \Gamma}}
$$

$$
\mathbb{P}\left(T_{\mathbf{e}}^{\tau,-}(\delta) \leq \tau \wedge S_{\mathbf{e}}^{\tau}(K)\right) \leq e^{-\delta^{2} / 2 K e^{B \Gamma}} .
$$

Letting $T_{\mathbf{e}}^{\tau}(\delta)=T_{\mathbf{e}}^{\tau,+}(\delta) \wedge T_{\mathbf{e}}^{\tau,-}(\delta)$, it follows that

$$
\mathbb{P}\left(T_{\mathbf{e}}^{\tau}(\delta) \leq \tau \wedge S_{\mathbf{e}}^{\tau}(K)\right) \leq 2 e^{-\delta^{2} / 2 K e^{B \Gamma}}
$$

Choosing $\Gamma=B^{-1} \log 2$ and $\delta=\sqrt{\omega K}$, with $0<\omega<4(\log 2)^{2} K / B^{2}$, we have $\|\theta\| \leq \Gamma$, so

$$
\mathbb{P}\left(\sup _{t \leq \tau \wedge S_{\mathbf{e}}^{\tau}(K)}\left|M_{\mathbf{e}}^{\tau}(t)\right|>\sqrt{\omega K}\right) \leq 2 e^{-\omega / 4} .
$$

We will now apply this to all times $\tau_{1}=\sigma, \tau_{2}=2 \sigma, \ldots, \tau_{\left\lceil e^{\omega / 8}\right\rceil}=\left\lceil e^{\omega / 8}\right\rceil \sigma$, up to time $\sigma\left\lceil e^{\omega / 8}\right\rceil$. Since $e^{\omega / 8} \geq 1$,

$$
\mathbb{P}\left(\exists j: T_{\mathbf{e}}^{\tau_{j}}(\sqrt{\omega K}) \leq \tau_{j} \wedge S_{\mathbf{e}}^{\tau_{j}}(K)\right) \leq 4 e^{-\omega / 8} .
$$


Now, for $t \leq \tau, M^{t}(t)=e^{\tilde{A}(t-\tau)} M^{\tau}(t)$, and so, $M_{\mathbf{e}}^{t}(t)=\mathbf{e}^{T} e^{\tilde{A}(t-\tau)} M^{\tau}(t)$. In particular, if $\mathbf{e}$ is an eigenvector of $\tilde{A}$ with eigenvalue $-\eta$, then $M_{\mathbf{e}}^{t}(t)=$ $e^{-\eta(t-\tau)} M_{\mathbf{e}}^{\tau}(t)$. In general,

$$
\begin{aligned}
M_{\mathbf{e}}^{t}(t) & =\mathbf{e}^{T} e^{\tilde{A}(t-\tau)} M^{\tau}(t) \leq\left\|\mathbf{e}^{T} e^{\tilde{A}(t-\tau)}\right\|\left\|M^{\tau}(t)\right\| \\
& \leq\left\|e^{\tilde{A}(t-\tau)}\right\| \sqrt{\sum_{i}\left(M_{i}^{\tau}(t)\right)^{2}} \leq e^{\nu(t-\tau)} \sqrt{\sum_{i}\left(M_{i}^{\tau}(t)\right)^{2}} \\
& \leq e^{\|\tilde{A}\|(t-\tau)} \sqrt{\sum_{i}\left(M_{i}^{\tau}(t)\right)^{2}} \leq e^{\min \left\{\|\tilde{A}\|_{1},\|\tilde{A}\|_{\infty}\right\} \sqrt{k}(t-\tau)} \sqrt{\sum_{i}\left(M_{i}^{\tau}(t)\right)^{2}},
\end{aligned}
$$

where $M_{i}^{\tau}(t)=M_{\mathbf{e}_{I}}^{\tau}(t)$ with $\mathbf{e}_{i}$ a unit vector with 1 in the $i$-th co-ordinate, $\nu$ is the largest eigenvalue of $\left(\tilde{A}+\tilde{A}^{T}\right) / 2,\|\tilde{A}\|_{\infty}=\max _{i} \sum_{j}\left|\tilde{A}_{i j}\right|$ and $\|\tilde{A}\|_{1}=$ $\max _{j} \sum_{i}\left|\tilde{A}_{i j}\right|$.

Choosing $\sigma<\tau$, if $\mathbf{e}$ is an eigenvector of $\tilde{A}$ with eigenvalue $-\eta$, then

$$
\sup _{\tau-\sigma \leq t \leq \tau}\left|M_{\mathbf{e}}^{t}(t)\right| \leq e^{\sigma \eta} \sup _{\tau-\sigma \leq t \leq \tau}\left|M_{\mathbf{e}}^{\tau}(t)\right| .
$$

For general e,

$$
\sup _{\tau-\sigma \leq t \leq \tau}\left|M_{\mathbf{e}}^{t}(t)\right| \leq e^{\min \left\{\|\tilde{A}\|_{1},\|\tilde{A}\|_{\infty}\right\} \sqrt{k} \sigma} \sqrt{\sum_{i}\left(M_{i}^{\tau}(t)\right)^{2}}
$$

From the above, since if $t<S_{\mathbf{e}}(K)$, then $S_{\mathbf{e}}^{t}(K)=\infty$, for $\mathbf{e}$ an eigenvector of $\tilde{A}$ with eigenvalue $\eta$,

$$
\mathbb{P}\left(\sup _{t \leq \sigma\left\lceil e^{\omega / 8}\right\rceil \wedge S_{\mathbf{e}}(K)}\left|M_{\mathbf{e}}^{t}(t)\right|>e^{\sigma \eta} \sqrt{\omega K}\right) \leq 4 e^{-\omega / 8} .
$$

Recalling that, given numbers $K_{1}, \ldots, K_{k}>0, S\left(K_{1}, \ldots, K_{k}\right)=\wedge_{i=1}^{k} S_{i}\left(K_{i}\right)$, we have, for a general vector $\mathbf{e}$,

$$
\mathbb{P}\left(\sup _{t \leq \sigma\left\lceil e^{\omega / 8}\right\rceil \wedge S\left(K_{1}, \ldots, K_{k}\right)}\left|M_{\mathbf{e}}^{t}(t)\right|>e^{\min \left\{\|\tilde{A}\|_{1},\|\tilde{A}\|_{\infty}\right\} \sqrt{k} \sigma} \sqrt{\omega} \sqrt{\sum_{i} K_{i}}\right) \leq 4 k e^{-\omega / 8} .
$$

Proof of Lemma 6. The possible jumps $y$ of $\left(\tilde{x}_{N}(t)\right)$ are of the form $\pm(1 / N, 0)^{T}$ and $\pm(1 / a N, 1 / N)^{T}$, so $\left\|e^{\tilde{A}(\tau-s)} y\right\| \leq B:=b / N$ for $s \leq \tau$, where $b=(|a|+1) /|a|$. 
EXTINCTION TIME FOR THE WEAKER OF TWO COMPETING SIS EPIDEMICS 23

We bound $\left.\int_{0}^{t} v_{i}\left(\tilde{x}_{N}(s), t-s\right) d s=\int_{0}^{t} \sum_{y} \tilde{q}_{N}\left(\tilde{x}_{N}(s), \tilde{x}_{N}(s)+y\right)\left(e^{\tilde{A}(t-s)} y\right)_{i}\right)^{2} d s:$

$$
\begin{aligned}
& \int_{0}^{t} v_{1}\left(\tilde{x}_{N}(s), t-s\right) d s \\
& \leq \frac{\lambda_{1}}{N} \int_{0}^{t} x_{N, 1}(s)\left(1-x_{N, 1}(s)-x_{N, 2}(s)\right) e^{-2 \eta_{1}(t-s)} d s \\
&+\frac{\mu_{1}}{N} \int_{0}^{t} x_{N, 1}(s) e^{-2 \eta_{1}(t-s)} d s+\frac{\mu_{2}}{N a^{2}} \int_{0}^{t} x_{N, 2}(s) e^{-2 \eta_{1}(t-s)} d s \\
&+\frac{\lambda_{2}}{N a^{2}} \int_{0}^{t} x_{N, 2}(s)\left(1-x_{N, 1}(s)-x_{N, 2}(s)\right) e^{-2 \eta_{1}(t-s)} d s \\
& \leq N^{-1}\left(\lambda_{1}+\mu_{1}\right) \int_{0}^{t} e^{-2 \eta_{1}(t-s)} d s+N^{-1} \frac{\left(\lambda_{2}+\mu_{2}\right)}{a^{2}} \int_{0}^{t} e^{-2 \eta_{1}(t-s)} d s \\
& \quad \leq N^{-1}\left(2 \eta_{1}\right)^{-1}\left[\left(\lambda_{1}+\mu_{1}\right)+a^{-2}\left(\lambda_{2}+\mu_{2}\right)\right] \leq \frac{a_{1}}{N} ; \\
& \int_{0}^{t} v_{2}\left(\tilde{x}_{N}(s), t-s\right) d s \\
& \leq \quad \frac{\lambda_{2}}{N} \int_{0}^{t} x_{N, 2}(s)\left(1-x_{N, 1}(s)-x_{N, 2}(s)\right) e^{-2 \eta_{2}(t-s)} d s+\frac{\mu_{2}}{N} \int_{0}^{t} x_{N, 2}(s) e^{-2 \eta_{2}(t-s)} d s \\
& \leq \quad N^{-1}\left(\lambda_{2}+\mu_{2}\right) \int_{0}^{t} e^{-2 \eta_{2}(t-s)} d s \leq N^{-1} \frac{\lambda_{2}+\mu_{2}}{2 \eta_{2}}=\frac{a_{2}}{N} .
\end{aligned}
$$

Now,

$$
\begin{aligned}
\left|\tilde{x}_{N, 2}(t)-\tilde{x}_{2}(t)\right| & \leq e^{-t \eta_{2}}\left|\tilde{x}_{N, 2}(0)-\tilde{x}_{2}(0)\right|+\left|\int_{0}^{t} e^{-(t-s) \eta_{2}} d M_{N, 2}(s) d s\right| \\
& +\frac{\lambda_{2}}{|a|} \frac{\eta_{2}}{\eta_{1}} \int_{0}^{t} e^{-(t-s) \eta_{2}}\left|\left(\tilde{x}_{N, 2}(s)\right)^{2}-\left(\tilde{x}_{2}(s)\right)^{2}\right| d s \\
& +\lambda_{2} \int_{0}^{t} e^{-(t-s) \eta_{2}}\left|\tilde{x}_{N, 1}(s) \tilde{x}_{N, 2}(s)-\tilde{x}_{1}(s) \tilde{x}_{2}(s)\right| d s
\end{aligned}
$$

and

$$
\begin{aligned}
& \left|\tilde{x}_{N, 1}(t)-\tilde{x}_{1}(t)\right| \\
& \quad \leq e^{-t \eta_{1}}\left|\tilde{x}_{N, 1}(0)-\tilde{x}_{1}(0)\right|+\left|\int_{0}^{t} e^{-(t-s) \eta_{1}} d M_{N, 1}(s) d s\right| \\
& \quad+\lambda_{1} \int_{0}^{t} e^{-(t-s) \eta_{1}}\left|\tilde{x}_{N, 1}(s)^{2}-\tilde{x}_{1}(s)^{2}\right| d s \\
& \quad+\frac{\eta_{2}\left|\lambda_{1}-\lambda_{2}\right|}{\eta_{1} a^{2}} \int_{0}^{t} e^{-(t-s) \eta_{1}}\left|\tilde{x}_{N, 2}(s)^{2}-\tilde{x}_{2}(s)^{2}\right| d s \\
& \quad+\frac{1}{|a|}\left(\left|\lambda_{1}-\lambda_{2}\right|+\frac{\lambda_{1} \eta_{2}}{\eta_{1}}\right) \int_{0}^{t} e^{-(t-s) \eta_{1}}\left|\tilde{x}_{N, 1}(s) \tilde{x}_{N, 2}(s)-\tilde{x}_{1}(s) \tilde{x}_{2}(s)\right| d s .
\end{aligned}
$$


Then, using Lemma 1,

$$
\begin{aligned}
& \frac{1}{|a|}\left|\tilde{x}_{N, 2}(t)-\tilde{x}_{2}(t)\right| \\
& \leq e^{-t \eta_{2}} f_{N}(0)+\left|\int_{0}^{t} e^{-(t-s) \eta_{2}} d M_{N, 2}(s) d s\right| \\
& \quad+\frac{\lambda_{2}}{|a|} \frac{\eta_{2}}{\eta_{1}} \int_{0}^{t} e^{-(t-s) \eta_{2}} f_{N}(s)\left(2 \tilde{x}_{2}(s)+|a| f_{N}(s)\right) d s \\
& +\frac{1}{|a|} \int_{0}^{t} e^{-(t-s) \eta_{2}} \lambda_{2} f_{N}(s)\left(\tilde{x}_{2}(s)+|a| f_{N}(s)+|a|\left|\tilde{x}_{1}(s)\right|\right) d s \\
& \leq e^{-t \eta_{2}} f_{N}(0)+\lambda_{2}\left(\frac{\eta_{2}}{\eta_{1}}+1\right) \int_{0}^{t}\left(f_{N}(s)\right)^{2} e^{-(t-s) \eta_{2}} d s \\
& \quad+\lambda_{2}\left(\frac{2 \eta_{2}}{\eta_{1}}+1\right) \int_{0}^{t} f_{N}(s) e^{-(t-s) \eta_{2}} \frac{1}{|a|} \tilde{x}_{2}(s) d s+\lambda_{2} \int_{0}^{t} f_{N}(s) e^{-(t-s) \eta_{2}}\left|\tilde{x}_{1}(s)\right| d s \\
& +\left|\int_{0}^{t} e^{-(t-s) \eta_{2}} d M_{N, 2}(s) d s\right| \\
& \leq e^{-t \eta_{2}} f_{N}(0)+L_{1} \int_{0}^{t}\left(f_{N}(s)\right)^{2} e^{-(t-s) \eta_{2}} d s+4 L_{1} y(0) e^{-t L} \int_{0}^{t} f_{N}(s) d s \\
& +\left|\int_{0}^{t} e^{-(t-s) \eta_{2}} d M_{N, 2}(s) d s\right|
\end{aligned}
$$

and

$$
\begin{aligned}
& \left|\tilde{x}_{N, 1}(t)-\tilde{x}_{1}(t)\right| \\
& \quad \leq \quad e^{-t \eta_{1}} f_{N}(0)+\left|\int_{0}^{t} e^{-(t-s) \eta_{1}} d M_{N, 1}(s) d s\right| \\
& \quad+\lambda_{1} \int_{0}^{t} e^{-(t-s) \eta_{1}} f_{N}(s)\left(2\left|\tilde{x}_{1}(s)\right|+f_{N}(s)\right) d s \\
& \quad+\frac{\left|\lambda_{1}-\lambda_{2}\right| \eta_{2}}{\eta_{1}} \int_{0}^{t} e^{-(t-s) \eta_{1}} f_{N}(s)\left(2 \frac{\tilde{x}_{2}(s)}{|a|}+f_{N}(s)\right) d s \\
& \quad+\left(\left|\lambda_{1}-\lambda_{2}\right|+\frac{\lambda_{1} \eta_{2}}{\eta_{1}}\right) \int_{0}^{t} e^{-(t-s) \eta_{1}} f_{N}(s)\left(\frac{\tilde{x}_{2}(s)}{|a|}+f_{N}(s)+\left|\tilde{x}_{1}(s)\right|\right) d s \\
& \quad \leq e^{-t \eta_{1}} f_{N}(0)+L_{1} \int_{0}^{t} e^{-(t-s) \eta_{1}}\left(f_{N}(s)\right)^{2} d s \\
& \quad+4 L_{1} e^{-t L} y(0) \int_{0}^{t} f_{N}(s) d s+\left|\int_{0}^{t} e^{-(t-s) \eta_{1}} d M_{N, 1}(s) d s\right|
\end{aligned}
$$

Let $T_{1}$ be the infimum of times $t$ such that

$$
\left|\int_{0}^{t} e^{-(t-s) \eta_{i}} d M_{N, i}(s) d s\right|>\left(\frac{\omega a_{i}}{N}\right)^{1 / 2} e^{\eta_{i}},
$$


EXTINCTION TIME FOR THE WEAKER OF TWO COMPETING SIS EPIDEMICS 25 for $i=1$ or $i=2$. On the event $t<T_{1}$,

$$
\begin{aligned}
\frac{\left|\tilde{x}_{N, 2}(t)-\tilde{x}_{2}(t)\right|}{|a|} & \leq e^{-t \eta_{2}} f_{N}(0)+L_{1} \int_{0}^{t} e^{-(t-s) \eta_{2}}\left(f_{N}(s)\right)^{2} d s \\
& +4 L_{1} y(0) e^{-t L} \int_{0}^{t} f_{N}(s) d s+\left(\frac{\omega a_{2}}{N}\right)^{1 / 2} e^{\eta_{2}}
\end{aligned}
$$

and

$$
\begin{aligned}
\left|\tilde{x}_{N, 1}(t)-\tilde{x}_{1}(t)\right| & \leq e^{-t \eta_{1}} f_{N}(0)+L_{1} \int_{0}^{t} e^{-(t-s) \eta_{1}}\left(f_{N}(s)\right)^{2} d s \\
& +4 L_{1} y(0) e^{-t L} \int_{0}^{t} f_{N}(s) d s+\left(\frac{\omega a_{1}}{N}\right)^{1 / 2} e^{\eta_{1}}
\end{aligned}
$$

Hence, for $t<T_{1}$,

$$
\begin{aligned}
f_{N}(t) \leq & e^{-t L} f_{N}(0)+L_{1} \int_{0}^{t} e^{-(t-s) L}\left(f_{N}(s)\right)^{2} d s \\
& +4 L_{1} y(0) e^{-t L} \int_{0}^{t} f_{N}(s) d s+\left(\frac{2 \omega\left(a_{1}+a_{2}\right)}{N}\right)^{1 / 2} e^{\tilde{L}} .
\end{aligned}
$$

Let $T_{2}=\inf \left\{t: f_{N}(t)>10 e^{\tilde{L}}\left(\frac{\omega\left(a_{1}+a_{2}\right.}{N}\right)^{1 / 2}\right\}$. Then on the event $t<T_{1} \wedge T_{2}$,

$$
\begin{aligned}
f_{N}(t) \leq & e^{-t L} f_{N}(0)+100 e^{2 \tilde{L}} \frac{L_{1}}{L} \frac{\omega\left(a_{1}+a_{2}\right)}{N}+4 L_{1} y(0) e^{-t L} \int_{0}^{t} f_{N}(s) d s \\
& +\left(\frac{2 \omega\left(a_{1}+a_{2}\right)}{N}\right)^{1 / 2} e^{\tilde{L}}
\end{aligned}
$$

so, for $N$ large enough,

$$
f_{N}(t) \leq e^{-t L} f_{N}(0)+4 L_{1} y(0) e^{-t L} \int_{0}^{t} f_{N}(s) d s+2\left(\frac{2 \omega\left(a_{1}+a_{2}\right)}{N}\right)^{1 / 2} e^{\tilde{L}} .
$$

Letting $g_{N}(t)=f_{N}(t) e^{t L}$, we see that, for large $N$, on the event $t<T_{1} \wedge T_{2}$,

$$
g_{N}(t) \leq g_{N}(0)+4 L_{1} y(0) \int_{0}^{t} e^{-s L} g_{N}(s) d s+2 e^{t L}\left(\frac{2 \omega\left(a_{1}+a_{2}\right)}{N}\right)^{1 / 2} e^{\tilde{L}} .
$$

By Grönwall's lemma, for large $N$, for $t<T_{1} \wedge T_{2}$,

$$
g_{N}(t) \leq\left(g_{N}(0)+2 e^{t L+\tilde{L}}\left(\frac{2 \omega\left(a_{1}+a_{2}\right)}{N}\right)^{1 / 2}\right) e^{\frac{4 L_{1} y(0)}{L}}
$$

so, if $y(0) \leq L / 8 L_{1}$ and $f_{N}(0) \leq e^{\tilde{L}}\left(\omega\left(a_{1}+a_{2}\right) / N\right)^{1 / 2}$, then

$$
f_{N}(t) \leq 2\left(f_{N}(0)+2 e^{\tilde{L}}\left(\frac{2 \omega\left(a_{1}+a_{2}\right)}{N}\right)^{1 / 2}\right) \leq 8 e^{\tilde{L}}\left(\frac{\omega\left(a_{1}+a_{2}\right)}{N}\right)^{1 / 2} .
$$

Fix $0<t_{0} \leq e^{\omega / 8}$. Let $T_{3}=\inf \left\{t: f_{N}(t)>8 e^{\tilde{L}}\left(\frac{\omega\left(a_{1}+a_{2}\right)}{N}\right)^{1 / 2}\right\}$. We now apply Lemma 7 to $\left(\tilde{x}_{N}(t)\right)$, with matrix $\tilde{A}$ as in (11) and $\tilde{F}$ as in (12), $B=b / N, \sigma=1$. We take $\eta$ to be equal to $\eta_{i}$ and $K$ to be equal to $K_{i}=a_{i} N^{-1}$, and $\mathbf{e}$ to be equal to $\mathbf{e}_{i}$, for $i=1,2$, and note that we have 
FABIO LOPES AND MALWINA LUCZAK

shown that $\left.\mathbb{P}\left(\int_{0}^{t} v_{i}\left(\tilde{x}_{N}(s), t-s\right) d s \leq K_{i} \quad i=1,2, \quad \forall t \leq\left\lceil e^{\omega / 8}\right\rceil\right\}\right)=1$. Lemma 7 then implies that $\mathbb{P}\left(T_{1} \leq\left\lceil e^{\omega / 8}\right\rceil\right) \leq 8 e^{-\omega / 8}$.

Also, since jumps are of size $O(1 / N), \mathbb{P}\left(T_{2} \leq T_{3}\right)=0$ for large $N$. Furthermore, we showed above that $\mathbb{P}\left(T_{3}<T_{1} \wedge T_{2}\right)=0$. Then we can only have $T_{3}<T_{1}$ if $T_{3} \geq T_{2}$, and hence $\mathbb{P}\left(T_{3}<T_{1}\right)=0$. It follows that

$\mathbb{P}\left(T_{3} \leq\left\lceil e^{\omega / 8}\right\rceil\right) \leq \mathbb{P}\left(T_{1} \leq\left\lceil e^{\omega / 8}\right\rceil\right)+\mathbb{P}\left(T_{3}<T_{1} \wedge T_{2}\right)+\mathbb{P}\left(T_{2} \leq T_{3}\right) \leq 8 e^{-\omega / 8}$, which completes the proof of Lemma 6 ,

Remark 1. When $a=0$, the matrix $A$ has a repeated eigenvalue, and is not diagonalisable. We instead work with the original variables $x_{N, 1}(t)$ and $x_{N, 2}(t)$. Letting $f_{N}(t)=\max \left\{\left|x_{N, 1}(t)-x_{1}(t)\right|,\left|x_{N, 2}(t)-x_{2}(t)\right|\right\}$, we can show, using Lemmas 3 and 4, and the fact that $\lambda_{2} e^{-\left(\lambda_{1}-\mu_{1}\right) t / 2}\left[\left(\lambda_{1}-\mu_{1}\right) t+\right.$ $\left.\lambda_{1} / \lambda_{2}\right] \leq 2\left(\lambda_{1}+\lambda_{2}\right)$ for all $t$, that

$$
\begin{aligned}
f_{N}(t) & \leq 2 e^{-\left(\lambda_{1}-\mu_{1}\right) t / 2} f_{N}(0)+4\left(\lambda_{1}+\lambda_{2}\right) \int_{0}^{t} e^{-\left(\lambda_{1}-\mu_{1}\right)(t-s) / 2} f_{N}(s)^{2} d s \\
& +32\left(\lambda_{1}+\lambda_{2}\right) y(0) e^{-\left(\lambda_{1}-\mu_{1}\right) t / 2} \int_{0}^{t} f_{N}(s) d s+M,
\end{aligned}
$$

where

$$
\begin{gathered}
M=\max \left\{\left|\int_{0}^{t}\left(e^{A(t-s)} d M_{N}(s)\right)_{1} d s\right|,\left|\int_{0}^{t}\left(e^{A(t-s)} d M_{N}(s)\right)_{2} d s\right|\right\}, \\
e^{u A}=\left(\begin{array}{cc}
e^{-u\left(\lambda_{1}-\mu_{1}\right)} & -\left(\lambda_{1}-\mu_{1}\right) u e^{-u\left(\lambda_{1}-\mu_{1}\right)} \\
0 & e^{-u\left(\lambda_{1}-\mu_{1}\right)}
\end{array}\right),
\end{gathered}
$$

$y(0)=\max \left\{\left|x_{1}(0)-\left(\lambda_{1}-\mu_{1}\right) / \lambda_{1}\right|, x_{2}(0)\right\}$. The remainder of the analysis can then be carried out in a way analogous to the case with distinct eigenvalues. We apply Lemma 7, taking $\mathbf{e}=\mathbf{e}_{i}$ for $i=1,2$, and noting that $\mathbf{e}_{2}$ is an eigenvector of $A$. We further take $B=3 / N, K_{1}=K_{2}=N^{-1}(2 \eta)^{-1}\left(\lambda_{1}+\mu_{1}+\lambda_{2}+\right.$ $\left.\mu_{2}\right)$, where $\eta=\eta_{1}=\eta_{2}=\lambda_{1}-\mu_{1}$. Then we can bound the size of the martingale deviation $M$ by $e^{3\left(\lambda_{1}-\mu_{1}\right) / 2} N^{-1 / 2} \sqrt{\omega\left(\lambda_{1}+\mu_{1}+\lambda_{2}+\mu_{2}\right) /\left(\lambda_{1}-\mu_{1}\right)}$, and hence we can show that

$$
f_{N}(t) \leq 8 e^{3\left(\lambda_{1}-\mu_{1}\right) / 2} N^{-1 / 2} \sqrt{\omega\left(\lambda_{1}+\mu_{1}+\lambda_{2}+\mu_{2}\right) /\left(\lambda_{1}-\mu_{1}\right)},
$$

provided $f_{N}(0) \leq e^{3\left(\lambda_{1}-\mu_{1}\right) / 2} N^{-1 / 2} \sqrt{\omega\left(\lambda_{1}+\mu_{1}+\lambda_{2}+\mu_{2}\right) /\left(\lambda_{1}-\mu_{1}\right)}$ and $y(0) \leq$ $\left(\lambda_{1}-\mu_{1}\right) / 128\left(\lambda_{1}+\lambda_{2}\right)$.

\section{Final Phase}

We will prove the following lemma.

Lemma 8. For $w \in \mathbb{R}$, let $t_{N}(y, w)=\left(\mu_{2}-\lambda_{2} \mu_{1} / \lambda_{1}\right)^{-1}(\log y+\log (1-$ $\left.\left.\lambda_{2} \mu_{1} / \lambda_{1} \mu_{2}\right)+w\right)$. Let $0<\varepsilon<1 / 4$. Suppose that $\left|x_{N, 1}(0)-\frac{\lambda_{1}-\mu_{1}}{\lambda_{1}}\right| \leq N^{-\varepsilon}$ and $\left|x_{N, 2}(0)-N^{-1 / 4}\right| \leq N^{-1 / 3}$. Then, as $N \rightarrow \infty$,

$$
\mathbb{P}\left(\kappa_{N} \leq t_{N}\left(N^{3 / 4}, w\right)\right) \rightarrow e^{-e^{-w}} .
$$


Let $(Y(t))_{t \geq 0}$ be a subcritical linear birth and death chain, with birth and death rates $\lambda$ and $\mu$, where $\mu>\lambda$. Let $T^{Y}=\inf \{t \geq 0: Y(t)=0\}$. It is a well known fact, see for example Renshaw (2011), that, for $t \geq 0$,

$$
\mathbb{P}\left(T^{Y} \leq t\right)=\mathbb{P}(Y(t)=0)=\left(1-\frac{(\mu-\lambda) e^{-(\mu-\lambda) t}}{\mu-\lambda e^{-(\mu-\lambda) t}}\right)^{Y(0)} .
$$

Assume that $Y(0)=y$, and let

$$
t(y, w)=\frac{\log y+\log (\mu-\lambda)-\log \mu+w}{\mu-\lambda},
$$

for $y$ and $w$ such that this is positive. Then $e^{-(\mu-\lambda) t(y, w)}=\mu e^{-w} /(\mu-\lambda) y$, so

$$
\mathbb{P}\left(T^{Y} \leq t(y, w)\right)=\left(1-\frac{e^{-w} / y}{1-\lambda e^{-w} /(\mu-\lambda) y}\right)^{y} .
$$

Now, consider a sequence $\left(Y_{N}(t)\right)$ of linear birth and death chains with birth rate $\lambda=\lambda(N)$ and death rate $\mu=\mu(N)$, where $\mu(N)>\lambda(N)$. Assume further that $Y_{N}(0)=y(N)$, where $y(\mu-\lambda) \rightarrow \infty$. Then

$$
\begin{aligned}
\mathbb{P}\left(T^{Y_{N}} \leq t(y(N), w)\right) & =\left(1-\frac{e^{-w} / y(N)}{1-\lambda e^{-w} /(\mu-\lambda) y(N)}\right)^{y(N)} \\
& =\left(1-\frac{e^{-w}}{y(N)-\lambda e^{-w} /(\mu-\lambda)}\right)^{y(N)} \rightarrow e^{-e^{-w}}
\end{aligned}
$$

as $N \rightarrow \infty$. In other words, the following result holds for the asymptotic distribution of the extinction times of a sequence of subcritical linear birth and death chains.

Lemma 9. Let $\left(Y_{N}(t)\right)$ be a sequence of subcritical linear birth and death chains with birth and death rates $\lambda(N)$ and $\mu(N)$, respectively, where $\mu(N)>$ $\lambda(N)$. Suppose further that $Y_{N}(0)=y(N)$, where $y(\mu-\lambda) \rightarrow \infty$. Let $T^{Y_{N}}=\inf \left\{t \geq 0: Y_{N}(t)=0\right\}$. Then, as $N \rightarrow \infty$,

$$
(\mu(N)-\lambda(N)) T^{Y_{N}}-(\log y(N)+\log (\mu(N)-\lambda(N))-\log \mu(N)) \rightarrow G,
$$

in distribution, where $G$ has a standard Gumbel distribution.

Proof of Lemma 8. Let $x_{1}(0)=x_{N, 1}(0)$ and $x_{2}(0)=x_{N, 2}(0)$, so $\mid x_{1}(0)-$ $\left(\lambda_{1}-\mu_{1}\right) / \lambda_{1} \mid \leq N^{-\varepsilon}$ and $x_{2}(0) \leq 2 N^{-1 / 4}$.

By Lemma 2, for large enough $N$, for all $t \geq 0, x_{2}(t) \leq 4 N^{-1 / 4}$. Also, by Lemma 1, if $N$ is large enough, for all $t \geq 0,\left|x_{1}(t)-\left(\lambda_{1}-\mu_{1}\right) / \lambda_{1}\right| \leq 4 N^{-\varepsilon}$.

Let $Z_{N}$ be a linear birth and death process defined as follows. The death rate is $\mu_{2}$, the birth rate is

$$
\lambda_{2}\left(1-\frac{\lambda_{1}-\mu_{1}}{\lambda_{1}}+5 N^{-\varepsilon}\right)=\frac{\lambda_{2} \mu_{1}}{\lambda_{1}}+5 \lambda_{2} N^{-\varepsilon},
$$


and $Z_{N}(0)=N^{3 / 4}+N^{2 / 3}$. By Lemma 9, as $N \rightarrow \infty$, in distribution,

$$
\begin{aligned}
\left(\mu_{2}\right. & \left.-\frac{\lambda_{2} \mu_{1}}{\lambda_{1}}-5 \lambda_{2} N^{-\varepsilon}\right) T^{Z_{N}} \\
& -\left(\log \left(N^{3 / 4}+N^{2 / 3}\right)+\log \left(\mu_{2}-\frac{\lambda_{2} \mu_{1}}{\lambda_{1}}-5 \lambda_{2} N^{-\varepsilon}\right)-\log \mu_{2}\right) \rightarrow G,
\end{aligned}
$$

where $G$ has a standard Gumbel distribution, and so, for $w \in \mathbb{R}$,

$\mathbb{P}\left(T^{Z_{N}} \leq \frac{\log \left(N^{3 / 4}+N^{2 / 3}\right)+\log \left(\mu_{2}-\frac{\lambda_{2} \mu_{1}}{\lambda_{1}}-5 \lambda_{2} N^{-\varepsilon}\right)-\log \mu_{2}+w}{\mu_{2}-\frac{\lambda_{2} \mu_{1}}{\lambda_{1}}-5 \lambda_{2} N^{-\varepsilon}}\right) \rightarrow e^{-e^{-w}}$.

This means that

$$
\mathbb{P}\left(T^{Z_{N}} \leq \frac{\log N^{3 / 4}+\log \left(\mu_{2}-\frac{\lambda_{2} \mu_{1}}{\lambda_{1}}\right)-\log \mu_{2}+w+o(1)}{\mu_{2}-\frac{\lambda_{2} \mu_{1}}{\lambda_{1}}}\right) \rightarrow e^{-e^{-w}},
$$

and so also

$$
\left(\mu_{2}-\frac{\lambda_{2} \mu_{1}}{\lambda_{1}}\right) T^{Z_{N}}-\left(\log N^{3 / 4}+\log \left(\mu_{2}-\frac{\lambda_{2} \mu_{1}}{\lambda_{1}}\right)-\log \mu_{2}\right) \rightarrow G .
$$

Let $W_{N}$ be a linear birth and death process defined as follows. The death rate is $\mu_{2}$, the birth rate is

$$
\lambda_{2}\left(1-\frac{\lambda_{1}-\mu_{1}}{\lambda_{1}}-6 N^{-\varepsilon}\right)=\frac{\lambda_{2} \mu_{1}}{\lambda_{1}}-6 \lambda_{2} N^{-\varepsilon},
$$

and $W_{N}(0)=N^{3 / 4}-N^{2 / 3}$. By Lemma 9, in distribution,

$$
\begin{gathered}
\left(\mu_{2}-\frac{\lambda_{2} \mu_{1}}{\lambda_{1}}+6 \lambda_{2} N^{-\varepsilon}\right) T^{W_{N}}-\log \left(N^{3 / 4}\left(1-N^{-1 / 12}\right)\right) \\
-\log \left(\mu_{2}-\frac{\lambda_{2} \mu_{1}}{\lambda_{1}}+6 \lambda_{2} N^{-\varepsilon}\right)+\log \mu_{2} \rightarrow G
\end{gathered}
$$

where $G$ has a standard Gumbel distribution. As above, it follows also that

$$
\left(\mu_{2}-\frac{\lambda_{2} \mu_{1}}{\lambda_{1}}\right) T^{W_{N}}-\left(\log N^{3 / 4}+\log \left(\mu_{2}-\frac{\lambda_{2} \mu_{1}}{\lambda_{1}}\right)-\log \mu_{2}\right) \rightarrow G .
$$

Let $f_{N}(t)$ be as in Lemma 6, and let $E_{t}$ be the event that $f_{N}(s) \leq N^{-1 / 3}$ for all $s<t$. For $N$ large enough, on the event $E_{t}$, for all $s<t$,

$$
-5 N^{-\varepsilon} \leq x_{N, 1}(s)-\frac{\lambda_{1}-1}{\lambda_{1}} \leq 5 N^{-\varepsilon},
$$

and, furthermore,

$$
0 \leq x_{N, 2}(s) \leq 4 N^{-1 / 4}+N^{-1 / 3} \leq N^{-\varepsilon} .
$$

Therefore, on the event $E_{t}$, we can couple $Z_{N}, W_{N}$ and $X_{N, 2}$ in such a way that, for $s \leq t$,

$$
W_{N}(s) \leq X_{N, 2}(s) \leq Z_{N}(s)
$$


It follows that, on the event $E_{t}, T^{Z_{N}} \leq t$ implies $\kappa_{N} \leq t$, and $\kappa_{N} \leq t$ implies $T^{W_{N}} \leq t$. Also, by Lemma 6 (with any $\omega=\omega(N)$ such that $\omega(N) / N^{1 / 3} \rightarrow 0$, $\mathbb{P}\left(\overline{E_{t}}\right) \rightarrow 0$ as long as $t \leq e^{\omega / 8}$. So, choosing $\omega(N)=N^{1 / 4}$, for $t \geq 0$,

$$
\mathbb{P}\left(\left\{T^{Z_{N}} \leq t\right\} \cap E_{t}\right) \leq \mathbb{P}\left(\left\{\kappa_{N} \leq t\right\} \cap E_{t}\right) \leq \mathbb{P}\left(\left\{T^{W_{N}} \leq t\right\} \cap E_{t}\right) .
$$

Hence, for any fixed $w$,

$$
\mathbb{P}\left(\kappa_{N} \leq t_{N}\left(N^{3 / 4}, w\right)\right) \leq \mathbb{P}\left(T^{W_{N}} \leq t_{N}\left(N^{3 / 4}, w\right)\right)+\mathbb{P}\left(\overline{E_{t_{N}\left(N^{3 / 4}, w\right)}}\right) \rightarrow e^{-e^{-w}},
$$

and

$$
\mathbb{P}\left(\kappa_{N} \leq t_{N}\left(N^{3 / 4}, w\right)\right) \geq \mathbb{P}\left(T^{Z_{N}} \leq t_{N}\left(N^{3 / 4}, w\right)\right)-\mathbb{P}\left(\overline{E_{t_{N}\left(N^{3 / 4}, w\right)}}\right) \rightarrow e^{-e^{-w}},
$$

which completes the proof of Lemma 8 ,

\section{Proof of Theorem 2}

By assumption, $x_{N}(0)=\left(\alpha_{N}, \beta_{N}\right)^{T}$, where $\alpha_{N} \rightarrow \alpha$ and $\beta_{N} \rightarrow \beta$ as $N \rightarrow$ $\infty$. We let $x(0)=x_{N}(0)$ as the initial condition for (11). By Theorem 3 and the discussion following it, if $\lambda_{1} / \mu_{1}>\lambda_{2} / \mu_{2}$ and $\lambda_{1} / \mu_{1}>1$, then the fixed point $x^{*}=\left(\frac{\lambda_{1}-\mu_{1}}{\lambda_{1}}, 0\right)^{T}$ of (1) is asymptotically stable, so that there exists $t_{0}>0$ such that, with $y(t)=e^{L t} \max \left\{\left\|\tilde{x}_{1}(t)\right\|, \tilde{x}_{2}(t)|a|^{-1}, L=\min \left\{\eta_{1}, \eta_{2}\right\}\right.$, as defined in Section 2.2. $\left|y\left(t_{0}\right)\right| \leq L / 8 L_{1}$. It is also not hard to see that we can choose a finite $t_{0}$ that works for every value of $N$, for $N$-dependent initial conditions as above.

Let $t_{N}=\inf \left\{t \geq t_{0}: x_{2}(t) \leq N^{-1 / 4}\right\}$. Lemma 2 implies that, as $N \rightarrow \infty$,

$$
t_{N}=\frac{1}{4 \eta_{2}} \log N+O(1) .
$$

It then also follows from Lemmas 1 and 2 that there exists $0<\varepsilon<1 / 4$ such that, if $N$ is large enough, then

$$
\left|x_{1}\left(t_{N}\right)-\frac{\lambda_{1}-\mu_{1}}{\lambda_{1}}\right| \leq \frac{1}{2} N^{-\varepsilon} .
$$

By Lemma 5 with $\delta=N^{-5 / 12}$ and Lemma 6 with $\omega=N^{1 / 4}$, if $N$ is large enough, then

$$
\begin{aligned}
& \sup _{t \leq t_{N}}\left|x_{N, 1}(t)-x_{1}(t)\right| \leq \frac{1}{2} N^{-1 / 3} \leq \frac{1}{2} N^{-\varepsilon} \\
& \sup _{t \leq t_{N}}\left|x_{N, 2}(t)-x_{2}(t)\right| \leq \frac{1}{2} N^{-1 / 3}
\end{aligned}
$$

with probability at least $1-e^{-N^{1 / 12}}$.

On the event that (14) holds, we can use Lemma 8 with $x_{N, 1}\left(t_{N}\right)$ and $x_{N, 2}\left(t_{N}\right)$ as initial values, since these values satisfy its hypotheses in this case. 
By (6), the length $t_{0}$ of the first phase can be written as

$$
\frac{\lambda_{2}}{\mu_{2} \lambda_{1}-\mu_{1} \lambda_{2}} \log \left(x_{1}\left(t_{0}\right) / x_{1}(0)\right)-\frac{\lambda_{1}}{\mu_{2} \lambda_{1}-\mu_{1} \lambda_{2}} \log \left(x_{2}\left(t_{0}\right) / x_{2}(0)\right),
$$

and the length $t_{N, 1}-t_{0}$ of the second phase can be written as

$$
\frac{\lambda_{2}}{\mu_{2} \lambda_{1}-\mu_{1} \lambda_{2}} \log \left(x_{1}\left(t_{N, 1}\right) / x_{1}\left(t_{0}\right)\right)-\frac{\lambda_{1}}{\mu_{2} \lambda_{1}-\mu_{1} \lambda_{2}} \log \left(x_{2}\left(t_{N, 1}\right) / x_{2}\left(t_{0}\right)\right) .
$$

On the event $\mathcal{E}\left(t_{N}\right)$ that (14) holds, by Lemma 8 , the length of the third phase is

$$
\frac{\lambda_{1}}{\mu_{2} \lambda_{1}-\mu_{1} \lambda_{2}}\left(\log N^{3 / 4}+\log \left(1-\frac{\mu_{1} \lambda_{2}}{\lambda_{1} \mu_{2}}\right)\right)+\frac{\lambda_{1}}{\mu_{2} \lambda_{1}-\mu_{1} \lambda_{2}} G_{N},
$$

where $G_{N}$ converges in distribution to a standard Gumbel variable $G$.

It follows that, on the event $\mathcal{E}\left(t_{N}\right)$, the total time $\kappa_{N}$ until the extinction of $X_{N, 2}$ is

$$
\begin{aligned}
& \frac{\lambda_{2}}{\mu_{2} \lambda_{1}-\mu_{1} \lambda_{2}} \log \left(x_{1}\left(t_{N, 1}\right) / \alpha_{N}\right)-\frac{\lambda_{1}}{\mu_{2} \lambda_{1}-\mu_{1} \lambda_{2}} \log \left(N^{-1 / 4} / \beta_{N}\right) \\
+ & \frac{\lambda_{1}}{\mu_{2} \lambda_{1}-\mu_{1} \lambda_{2}}\left(\log N^{3 / 4}+\log \left(1-\frac{\mu_{1} \lambda_{2}}{\mu_{2} \lambda_{1}}\right)\right)+\frac{\lambda_{1}}{\mu_{2} \lambda_{1}-\mu_{1} \lambda_{2}} G_{N} .
\end{aligned}
$$

Since $\mathbb{P}\left(\mathcal{E}\left(t_{N, 1}\right)\right) \rightarrow 1, \alpha_{N} \rightarrow \alpha, \beta_{N} \rightarrow \beta$ as $N \rightarrow \infty$, and, for large $N$, $\left|x_{1}\left(t_{N, 1}\right)-\left(\lambda_{1}-\mu_{1}\right) / \lambda_{1}\right| \leq N^{-\varepsilon}$, for an $\varepsilon>0$, we conclude

$$
\frac{\mu_{2} \lambda_{1}-\mu_{1} \lambda_{2}}{\lambda_{1}} \kappa_{N}-\left(\log N \beta\left(1-\frac{\mu_{1} \lambda_{2}}{\mu_{2} \lambda_{1}}\right)+\frac{\lambda_{2}}{\lambda_{1}} \log \left(\frac{1-\mu_{1} / \lambda_{1}}{\alpha}\right)\right) \rightarrow G,
$$

in distribution, where $G$ is a standard Gumbel, so our proof of the first part of Theorem 2 is complete.

The second part of Theorem 2 follows using Theorem 1, since, after the extinction of the weaker species, the stronger species evolves as a single supercritical logistic epidemic, and we have shown that $\kappa_{N}$ is with high probability negligible in comparison with $\tau_{N}$.

\section{NEAR-CRITICAL PHENOMENA}

In this section, we will show that Theorem 2 can be extended to nearcriticality. As a proof of concept, we consider the following special case where $\mu_{1}=\mu_{2}=1, \lambda_{1}>\lambda_{2}>1$, and $\lambda_{1}-\lambda_{2}=\lambda_{1}(N)-\lambda_{2}(N) \rightarrow 0$ as $N \rightarrow \infty$ (while $\lambda_{1}=\lambda_{1}(N)$ may or may not tend to 1 ). This may for example model a real-world scenario where a slightly more infectious strain emerges during an outbreak, for instance, via a mutation, and we want to know the time taken for it to supplant the weaker one in the population.

We assume that $\left(\lambda_{1}-\lambda_{2}\right)\left(\lambda_{1}-1\right)^{-1} \rightarrow 0$. We further assume that

$$
N\left(\lambda_{1}-\lambda_{2}\right)^{3}\left(\lambda_{1}-1\right)^{-1}\left(\log \log \left(N\left(\lambda_{1}-\lambda_{2}\right)^{2}\right)\right)^{-1} \rightarrow \infty .
$$

We believe that the last condition is not best possible, and that it is only necessary to have $N\left(\lambda_{1}-\lambda_{2}\right)^{2} \rightarrow \infty$ for the formula in Theorem 4 to hold. 
Note that under our assumptions $\left(\lambda_{2}-1\right)\left(\lambda_{1}-1\right)^{-1} \rightarrow 1$. Also, the quantity $a$ defined in (8) satisfies $0<1 \leq a$ for $N$ large enough, and converges to 1 as $N \rightarrow \infty$. As before, we assume that $X_{N, 1}(0)=\alpha_{N}, X_{N, 2}(0)=\beta_{N}$, where $\alpha_{N} \rightarrow \alpha>0$ and $\beta_{N} \rightarrow \beta>0$. We will prove the following result, which is an extension of Theorem 2 to this case.

Theorem 4. Under the above assumptions,

$$
\kappa_{N}=\frac{\lambda_{1}}{\lambda_{1}-\lambda_{2}}\left(\log \left(N \frac{\left(\lambda_{1}-1\right)\left(\lambda_{1}-\lambda_{2}\right)}{\lambda_{1}^{2}} \frac{\beta}{\alpha}\right)+G_{N}\right),
$$

where $G_{N}$ is a random variable converging in distribution to a Gumbel random variable $G$.

To prove Theorem 4, we first prove long-term estimates on the total number of infectives of either type and on the ratio between the number of infectives of the two types.

Let $\omega=\omega(N)>0$ satisfy $N\left(\lambda_{2}-1\right)^{2} / \omega \rightarrow \infty$ as $N \rightarrow \infty$. (Note that our assumptions imply that $N\left(\lambda_{2}-1\right)^{2} \rightarrow \infty$.) We will show that, with high probability (i.e with probability tending to 1 as $N \rightarrow \infty$ ), $x_{N, 1}(t)+x_{N, 2}(t) \geq$ $\left(\lambda_{2}-1\right) / 2 \lambda_{2}$ for all $t \leq\left(\lambda_{2}-1\right)^{-1} e^{\omega / 8}$.

Lemma 10. Let $Y_{N}(t)$ denote the number of infectives in a stochastic logistic SIS epidemic with infection rate $\lambda_{2}$ and recovery rate 1 . Suppose that $X_{N, 1}(0)+X_{N, 2}(0) \geq Y_{N}(0)$. Then, for all $t, X_{N, 1}(t)+X_{N, 2}(t)$ stochastically dominates $Y_{N}(t)$.

Further, let $Z_{N}(t)$ denote the number of infectives in a stochastic SIS logistic epidemic with infection rate $\lambda_{1}$ and recovery rate 1 . Suppose that $X_{N, 1}(0)+X_{N, 2}(0) \leq Z_{N}(0)$. Then, for all $t, X_{N, 1}(t)+X_{N, 2}(t)$ is stochastically dominated by $Z_{N}(t)$.

Proof. The process $X_{N, 1}(t)+X_{N, 2}(t)$ jumps by +1 at rate at least $\lambda_{2}\left(X_{N, 1}+\right.$ $\left.X_{N, 2}\right)\left(1-X_{N, 1}-X_{N, 2}\right)$ and jumps by -1 at rate $X_{N, 1}+X_{N, 2}$. We can then couple $X_{N, 1}+X_{N, 2}$ and $Y_{N}(t)$ so that they always jump down together as much as possible, and jump up together as much as possible, and otherwise each jumps on its own with any excess rate in either direction. With this coupling, $X_{N, 1}(t)+X_{N, 2}(t) \geq Y_{N}(t)$. The second part can be proved analogously.

Lemma 11. Let $Y_{N}(t)$ denote the number of infective individuals in a stochastic SIS logistic epidemic with infection rate $\lambda=\lambda(N)$ and recovery rate $\mu=\mu(N)$. Let $y(t)$ denote the number of infectives in the corresponding deterministic SIS logistic epidemic. We assume that $\lambda=\lambda(N)$ and $\mu=\mu(N)$, where $\lambda, \mu$ are bounded, $\lambda>\mu>0$, and $(\lambda(N)-\mu(N))^{2} N \rightarrow \infty$ as $N \rightarrow \infty$. Let $\omega=\omega(N)>0$ satisfy $N(\lambda-\mu)^{2} / \omega \rightarrow \infty$ as $N \rightarrow \infty$.

We assume that $y(0):=y_{N} \leq 2(\lambda-\mu) / \lambda$, and $y_{N} /(\lambda-\mu)$ is bounded away from 0 as $N \rightarrow \infty$. Further, let $Y_{N}(0)$ be such that $\left|N^{-1} Y_{N}(0)-y(0)\right| \leq$ 
$\frac{1}{2} \sqrt{\frac{2 \omega(\lambda+\mu)}{N \lambda}}$. If $y_{N} \geq(\lambda-\mu) / \lambda$, then, for $N$ sufficiently large,

$$
\mathbb{P}\left(\sup _{t \leq(\lambda-\mu)^{-1} e^{\omega / 8}}\left|N^{-1} Y_{N}(t)-y(t)\right|>4 e^{4} \sqrt{\frac{2 \omega(\lambda+\mu)}{N \lambda}}\right) \leq 4 e^{-\omega / 8} .
$$

If $y_{N}<(\lambda-\mu) / \lambda$, then, for $N$ sufficiently large,

$\mathbb{P}\left(\sup _{t \leq(\lambda-\mu)^{-1} e^{\omega / 8}}\left|N^{-1} Y_{N}(t)-y(t)\right|>4 e^{4(\lambda-\mu) / \lambda y(0)} \sqrt{\frac{2 \omega(\lambda+\mu)}{N \lambda}}\right) \leq 4 e^{-\omega / 8}$.

Suppose further that $(\lambda-\mu) / 2 \lambda \leq y(0)$. Then, in particular, since $N(\lambda-$ $\mu)^{2} / \omega \rightarrow \infty, y_{N}(t)=N^{-1} Y_{N}(t)$ remains well concentrated around $y(t)$ until time at least $(\lambda-\mu)^{-1} e^{\omega / 8}$. Consequently, if $N$ is sufficiently large, $(\lambda-\mu) / 4 \lambda \leq y_{N}(t) \leq 4(\lambda-\mu) / \lambda$ for all $t \leq(\lambda-\mu)^{-1} e^{\omega / 8}$ with probability at least $1-4 e^{-\omega / 8}$.

Proof. Note that $(\lambda-\mu) / \lambda$ is an attractive fixed point for

$$
\frac{d y(t)}{d t}=\lambda y(t)(1-y(t))-\mu y(t)
$$

so $y(t) \rightarrow(\lambda-\mu) / \lambda$.

Let $\tilde{y}(t)=y(t)-(\lambda-\mu) / \lambda$ and let $\tilde{y}_{N}(t)=N^{-1} Y_{N}(t)-(\lambda-\mu) / \lambda$. Then

$$
\tilde{y}(t)=\tilde{y}(0)-(\lambda-\mu) \int_{0}^{t} \tilde{y}(s) d s-\lambda \int_{0}^{t} \tilde{y}(s)^{2} d s
$$

and

$$
\tilde{y}_{N}(t)=\tilde{y}_{N}(0)-(\lambda-\mu) \int_{0}^{t} \tilde{y}_{N}(s) d s-\lambda \int_{0}^{t} \tilde{y}_{N}(s)^{2} d s+m_{N}(t)
$$

where $\left(m_{N}(t)\right)$ is a zero-mean martingale.

It follows that

$$
\tilde{y}(t)=e^{-(\lambda-\mu) t} \tilde{y}(0)-\lambda \int_{0}^{t} e^{-(\lambda-\mu)(t-s)} \tilde{y}(s)^{2} d s,
$$

and

$\tilde{y}_{N}(t)=e^{-(\lambda-\mu) t} \tilde{y}_{N}(0)-\lambda \int_{0}^{t} e^{-(\lambda-\mu)(t-s)} \tilde{y}_{N}(s)^{2} d s+\int_{0}^{t} e^{-(\lambda-\mu)(t-s)} d m_{N}(s)$.

Letting $f_{N}(t)=\left|\tilde{y}(t)-\tilde{y}_{N}(t)\right|=\left|y(t)-y_{N}(t)\right|$, we thus have

$f_{N}(t) \leq f_{N}(0)+\lambda \int_{0}^{t} e^{-(\lambda-\mu)(t-s)} f_{N}(s)\left|\tilde{y}_{N}(s)+\tilde{y}(s)\right| d s+\left|\int_{0}^{t} e^{-(\lambda-\mu)(t-s)} d m_{N}(s)\right|$,

and so

$$
\begin{aligned}
f_{N}(t) & \leq f_{N}(0)+\lambda \int_{0}^{t} e^{-(\lambda-\mu)(t-s)}\left(f_{N}(s)\right)^{2} d s+2 \lambda \int_{0}^{t} e^{-(\lambda-\mu)(t-s)} f_{N}(s)|\tilde{y}(s)| d s \\
& +\left|\int_{0}^{t} e^{-(\lambda-\mu)(t-s)} d m_{N}(s)\right| .
\end{aligned}
$$


To estimate the deviations of the martingale transform $\int_{0}^{t} e^{-(\lambda-\mu)(t-s)} d m_{N}(s)$, we let $T_{1}=\inf \left\{t: y_{N}(t)>2 y(t)\right\}$; on the event $t<T_{1}$,

$$
\begin{aligned}
\int_{0}^{t} v\left(\tilde{y}_{N}(s), t-s\right):= & \frac{\lambda}{N} \int_{0}^{t} y_{N}(s)\left(1-y_{N}(s)\right) e^{-2(\lambda-\mu)(t-s)} d s \\
& +\frac{\mu}{N} \int_{0}^{t} y_{N}(s) e^{-2(\lambda-\mu)(t-s)} d s \\
\leq & \frac{2(\lambda+\mu)}{N} \int_{0}^{t} e^{-2(\lambda-\mu)(t-s)} y(s) d s .
\end{aligned}
$$

As is well known,

$$
y(t)=\frac{y(0)(\lambda-\mu)}{\lambda y(0)+\lambda e^{-(\lambda-\mu) t}\left(\frac{\lambda-\mu}{\lambda}-y(0)\right)},
$$

and so

$$
\tilde{y}(t)=\frac{\tilde{y}(0) e^{-(\lambda-\mu) t}}{y(0) \frac{\lambda}{\lambda-\mu}\left(1-e^{-(\lambda-\mu) t}\right)+e^{-(\lambda-\mu) t}} .
$$

We restrict attention for the moment to the case where $y(0) \geq(\lambda-\mu) / \lambda$, when we have $\tilde{y}(t) \leq \tilde{y}(0) e^{-(\lambda-\mu) t}$, and $(\lambda-\mu) / \lambda \leq y(t) \leq y(0) \leq 2(\lambda-\mu) / \lambda$. (If $y(0) \leq(\lambda-\mu) / \lambda$, then we have $\tilde{y}(t) \leq \frac{\lambda-\mu}{\lambda y(0)} \tilde{y}(0) e^{-(\lambda-\mu) t}$, and $y(0) \leq$ $y(t) \leq(\lambda-\mu) / \lambda$. $)$ It follows that, on the event $t<T_{1}$,

$$
\int_{0}^{t} v\left(\tilde{y}_{N}(s), t-s\right) \leq \frac{2(\lambda+\mu)}{N \lambda} .
$$

Given $\omega=\omega(N)>0$, let $T_{2}$ be the infimum of times $t$ such that

$$
\left|\int_{0}^{t} e^{-(\lambda-\mu)(t-s)} d m_{N}(s)\right|>3 \sqrt{\frac{2 \omega(\lambda+\mu)}{N \lambda}} .
$$

By Lemma77applied to $\left(y_{N}(t)\right)$, with $B=1 / N, \tilde{A}=-(\lambda-\mu), \sigma=1 /(\lambda-\mu)$, $\eta=\lambda-\mu, K=\frac{2(\lambda+\mu)}{N \lambda}$, we see that, if $\omega \leq 8(\log 2)^{2} N(\lambda+\mu) / \lambda$ (which will hold for $N$ large enough if $\left.N(\lambda-\mu)^{2} / \omega \rightarrow \infty\right)$ and $(\lambda-\mu)^{-1}\left\lceil e^{\omega / 8}\right\rceil \geq t_{0}=$ $t_{0}(N)$, then

$$
\mathbb{P}\left(T_{2} \leq T_{1} \wedge t_{0}\right) \leq 4 e^{-\omega / 8}
$$

Also, by the above, and using the assumption that $f_{N}(0) \leq \frac{1}{2} \sqrt{\frac{2 \omega(\lambda+\mu)}{N \lambda}}$, on the event $t_{0}<T_{1} \wedge T_{2}$, for all $t \leq t_{0}$,

$$
\begin{aligned}
f_{N}(t) \leq & \lambda \int_{0}^{t} e^{-(\lambda-\mu)(t-s)}\left(f_{N}(s)\right)^{2} d s+2 \lambda \int_{0}^{t} e^{-(\lambda-\mu)(t-s)} f_{N}(s)|\tilde{y}(s)| d s \\
& +\frac{7}{2} \sqrt{\frac{2 \omega(\lambda+\mu)}{N \lambda}} .
\end{aligned}
$$


Let $T_{3}$ be the infimum of times $t$ such that $f_{N}(t)>5 e^{4} \sqrt{\frac{2 \omega(\lambda+\mu)}{N \lambda}}$. Then, if $N$ is large enough, on the event $t_{0}<T_{1} \wedge T_{2} \wedge T_{3}$, for $t \leq t_{0}$,

$f_{N}(t) \leq \frac{\lambda}{\lambda-\mu} \frac{50 e^{8} \omega(\lambda+\mu)}{N \lambda}+2 \lambda \int_{0}^{t} e^{-(\lambda-\mu)(t-s)} f_{N}(s)|\tilde{y}(s)| d s+\frac{7}{2} \sqrt{\frac{2 \omega(\lambda+\mu)}{N \lambda}}$.

Since $N(\lambda-\mu)^{2} / \omega \rightarrow \infty$, then, for $N$ large enough,

$$
\frac{\lambda}{\lambda-\mu} \frac{50 e^{8} \omega(\lambda+\mu)}{N} \leq \frac{1}{2} \sqrt{\frac{2 \omega(\lambda+\mu)}{N \lambda}},
$$

and so, for $N$ large enough, on the event $t_{0}<T_{1} \wedge T_{2} \wedge T_{3}$, for $t \leq t_{0}$,

$$
f_{N}(t) e^{(\lambda-\mu) t} \leq 2 \lambda \int_{0}^{t} e^{(\lambda-\mu) s} f_{N}(s)|\tilde{y}(s)| d s+4 e^{(\lambda-\mu) t} \sqrt{\frac{2 \omega(\lambda+\mu)}{N \lambda}} .
$$

From (18), $\tilde{y}(t) \leq e^{-(\lambda-\mu) t} \tilde{y}(0) \leq 2 e^{-(\lambda-\mu) t}(\lambda-\mu) / \lambda$, so, by Grönwall's inequality, on the event $t_{0}<T_{1} \wedge T_{2} \wedge T_{3}$, for $t \leq t_{0}$,

$$
f_{N}(t) \leq 4 \sqrt{\frac{2 \omega(\lambda+\mu)}{N \lambda}} e^{4} .
$$

Letting $T_{4}$ be the infimum of $t$ with $f_{N}(t)>4 \sqrt{\frac{2 \omega(\lambda+\mu)}{N \lambda}} e^{4}$, from the above

$$
\mathbb{P}\left(T_{4} \leq t_{0}\right) \leq \mathbb{P}\left(T_{1} \wedge T_{2} \wedge T_{3} \leq T_{4} \wedge t_{0}\right),
$$

and so, since $\mathbb{P}\left(T_{3} \leq T_{4}\right)=0$ and $\mathbb{P}\left(T_{1} \leq T_{4}\right)=0$ for $N$ large enough,

$$
\mathbb{P}\left(T_{4} \leq t_{0}\right) \leq \mathbb{P}\left(T_{1} \leq T_{4}\right)+\mathbb{P}\left(T_{3} \leq T_{4}\right)+\mathbb{P}\left(T_{2} \leq T_{1} \wedge t_{0}\right) \leq 4 e^{-\omega / 8},
$$

and so, as claimed, for $N$ large enough,

$$
\mathbb{P}\left(\sup _{t \leq(\lambda-\mu)^{-1}\left\lceil e^{\omega / 8}\right\rceil}\left|y_{N}(t)-y(t)\right|>4 e^{4} \sqrt{\frac{2 \omega(\lambda+\mu)}{N \lambda}}\right) \leq 4 e^{-\omega / 8},
$$

and the remaining conclusions also follow in the case when $y(0) \geq(\lambda-\mu) / \lambda$.

The case $y(0) \leq(\lambda-\mu) / \lambda$ is similar, using the inequality from (18) that

$$
|\tilde{y}(t)| \leq \frac{|\tilde{y}(0)|(\lambda-\mu)}{y(0) \lambda} e^{-(\lambda-\mu) t}
$$

Lemma 12. Let $Y_{N}(t)$ denote the number of infective individuals in a stochastic SIS logistic epidemic with infection rate $\lambda=\lambda(N)$ and recovery rate $\mu=\mu(N)$. Let $y(t)$ denote the number of infectives in the corresponding deterministic SIS logistic epidemic. We assume that $\lambda=\lambda(N)$ and $\mu=\mu(N)$, where $\lambda, \mu$ are bounded, $\lambda>\mu>0$, and $(\lambda(N)-\mu(N))^{2} N \rightarrow \infty$ as $N \rightarrow \infty$.

We further assume that $y_{N}(0)=N^{-1} Y_{N}(0)>2(\lambda-\mu) / \lambda$, and we let $y(0)=y_{N}(0)$. Let $\tau$ be such that $y(\tau)=2(\lambda-\mu) / \lambda$. Then, for $N$ sufficiently large,

$\mathbb{P}\left(\sup _{t \leq \tau}\left|y_{N}(t)-y(t)\right|>2\left(N(\lambda-\mu)^{2}\right)^{1 / 16} \sqrt{\frac{y_{N}(0)(\lambda+\mu)}{N(\lambda-\mu)}}\right) \leq 2 e^{-\left(N(\lambda-\mu)^{2}\right)^{1 / 8} / 8}$. 
EXTINCTION TIME FOR THE WEAKER OF TWO COMPETING SIS EPIDEMICS 35

Proof. We have

$$
\begin{aligned}
y_{N}(t)-y(t)= & \left(y_{N}(0)-y(0)\right)-\lambda \int_{0}^{t}\left(y_{N}(s)-y(s)\right)\left(y_{N}(s)+y(s)-\frac{\lambda-\mu}{\lambda}\right) d s \\
& +m_{N}(s) .
\end{aligned}
$$

For $t \leq \tau, y(t) \geq 2(\lambda-\mu) / \lambda$ and so $y_{N}(s)+y(s)-(\lambda-\mu) / \lambda \geq 0$. By Lemma 3.2 in Brightwell, House and Luczak (2018),

$$
\sup _{t \leq \tau}\left|y_{N}(t)-y(t)\right| \leq 2\left|y_{N}(0)-y(0)\right|+2 \sup _{t \leq \tau}\left|m_{N}(s)\right| \text {. }
$$

From (17),

$$
e^{(\lambda-\mu) \tau}-1=1-\frac{2(\lambda-\mu)}{\lambda y(0)}
$$

Arguing as in the proof of Lemma 3.1 in Brightwell, House and Luczak (2018), using standard martingale techniques and the fact that

$$
\int_{0}^{t} y(s) d s=\frac{1}{\lambda} \log \left(\lambda y(0)\left(e^{\left(\lambda_{\mu}\right) t}-1\right)+(\lambda-\mu)\right)-\frac{1}{\lambda} \log (\lambda-\mu),
$$

we see that, if $\phi=\phi(N) \leq N^{1 / 2}$, then

$$
\mathbb{P}\left(\sup _{t \leq \tau}\left|y_{N}(t)-y(t)\right|>2 \sqrt{y_{N}(0) \phi(\lambda+\mu) / N(\lambda-\mu)}\right) \leq 2 e^{-\phi / 8}
$$

and so, taking $\phi=\left(N(\lambda-\mu)^{2}\right)^{1 / 8}$,

$$
\mathbb{P}\left(\sup _{t \leq \tau}\left|y_{N}(t)-y(t)\right|>2\left(N(\lambda-\mu)^{2}\right)^{1 / 16} \sqrt{\frac{y_{N}(0)(\lambda+\mu)}{N(\lambda-\mu)}}\right) \leq 2 e^{-\left(N(\lambda-\mu)^{2}\right)^{1 / 8} / 8} .
$$

Next we consider the ratio $Q\left(X_{N}(t)\right)=X_{N, 1}(t) / X_{N, 2}(t)$. We will show that the value of $Q\left(X_{N}(t)\right)$ does not change very much over a time period of length 'nearly' $\left(\lambda_{1}-\lambda_{2}\right)^{-1}$.

Lemma 13. Let $\psi=\psi(N) \rightarrow \infty$ in such a way that $\psi\left(\lambda_{1}-\lambda_{2}\right) \rightarrow 0$ as $N \rightarrow \infty$. Let $t_{0}=\left(\lambda_{1}-\lambda_{2}\right)^{-1} \psi(N)^{-1}$. Then, for $N$ large enough, $\mathbb{P}\left(\sup _{t \leq t_{0}}\left|\frac{X_{N, 1}(t)}{X_{N, 2}(t)}-\frac{X_{N, 1}(0)}{X_{N, 2}(0)}\right|>2 \psi(N)^{-1 / 4}\right) \leq 2 e^{-\psi(N)^{1 / 2} \beta^{2} / 128 \alpha(\alpha+\beta)}+4 e^{-\sqrt{N}\left(\lambda_{2}-1\right)}$.

Proof. Given a vector $X=\left(X_{1}, X_{2}\right)^{T}$ with non-negative integer components, such that $X_{2}>0$ and $X_{1}+X_{2} \leq N$, the drift $g_{N}(X)$ in $Q(X)$ is

$$
\begin{array}{r}
\frac{1}{X_{2}} \lambda_{1} X_{1}\left(1-\frac{X_{1}}{N}-\frac{X_{2}}{N}\right)-\frac{X_{1}}{X_{2}} \\
+X_{1}\left(\frac{1}{X_{2}+1}-\frac{1}{X_{2}}\right) \lambda_{2} X_{2}\left(1-\frac{X_{1}}{N}-\frac{X_{2}}{N}\right)+X_{1}\left(\frac{1}{X_{2}-1}-\frac{1}{X_{2}}\right) X_{2} \\
=\left(1-\frac{X_{1}}{N}-\frac{X_{2}}{N}\right)\left(\frac{\lambda_{1} X_{1}}{X_{2}}-\frac{\lambda_{2} X_{1}}{X_{2}+1}\right)-\frac{X_{1}}{X_{2}}+\frac{X_{1}}{X_{2}-1} .
\end{array}
$$


Clearly, we see that $g_{N}\left(X_{N}(t)\right) \geq 0$ for all $t$ at most the weaker species extinction time $\kappa_{N}$. Also,

$$
\begin{aligned}
g_{N}(X) & =\frac{X_{1}}{X_{2}}\left(\lambda_{1}-\lambda_{2}\right)+\lambda_{2} X_{1}\left(\frac{1}{X_{2}}-\frac{1}{X_{2}+1}\right)-X_{1}\left(\frac{1}{X_{2}}-\frac{1}{X_{2}-1}\right) \\
& +\left(\frac{X_{1}}{N}+\frac{X_{2}}{N}\right) X_{1}\left(\frac{\lambda_{2}}{X_{2}+1}-\frac{\lambda_{1}}{X_{2}}\right) \\
& \leq \frac{X_{1}}{X_{2}}\left(\lambda_{1}-\lambda_{2}\right)+\lambda_{2} X_{1}\left(\frac{1}{X_{2}}-\frac{1}{X_{2}+1}\right)-X_{1}\left(\frac{1}{X_{2}}-\frac{1}{X_{2}-1}\right) \\
& \leq \frac{X_{1}}{X_{2}}\left(\lambda_{1}-\lambda_{2}+\frac{\lambda_{2}}{X_{2}+1}+\frac{1}{X_{2}-1}\right)
\end{aligned}
$$

Let $T_{1}$ be the infimum of times $t$ such that $X_{N, 2}(t)-1<N\left(\lambda_{1}-\lambda_{2}\right)$. Then, since $N\left(\lambda_{1}-\lambda_{2}\right)^{2} \rightarrow \infty, 0 \leq g_{N}\left(X_{N}(t)\right) \leq 3 Q\left(X_{N}(t)\right)\left(\lambda_{1}-\lambda_{2}\right)$ for $t<T_{1}$, if $N$ is large enough.

We write $Q\left(X_{N}(t)\right)=Q\left(X_{N}(0)\right)+\int_{0}^{t} g_{N}\left(X_{N}(s)\right) d s+M_{N}(t)$, where $M_{N}(t)$ is a martingale. Let $R_{N}(X)$ be given by

$$
\begin{array}{r}
\frac{\lambda_{1} X_{1}}{X_{2}^{2}}\left(1-\frac{X_{1}}{N}-\frac{X_{2}}{N}\right)+\frac{X_{1}}{X_{2}^{2}}+X_{1}^{2}\left(\frac{1}{X_{2}+1}-\frac{1}{X_{2}}\right)^{2} \lambda_{2} X_{2}\left(1-\frac{X_{1}}{N}-\frac{X_{2}}{N}\right) \\
+X_{1}^{2}\left(\frac{1}{X_{2}-1}-\frac{1}{X_{2}}\right)^{2} X_{2} .
\end{array}
$$

Let $T_{2}$ be the infimum of times $t$ such that $Q\left(X_{N}(t)\right)>2 Q\left(X_{N}(0)\right)$. For $t<T_{1} \wedge T_{2}$, if $N$ is large enough,

$$
\begin{aligned}
R_{N}\left(X_{N}(t)\right) \leq & 6 Q\left(X_{N}(0)\right)\left(\lambda_{1}-\lambda_{2}\right)+4\left(Q\left(X_{N}(0)\right)\right)^{2}\left(\lambda_{1}-\lambda_{2}\right) \\
& +4\left(Q\left(X_{N}(0)\right)\right)^{2}\left(\lambda_{1}-\lambda_{2}\right) \\
\leq & 8 Q\left(X_{N}(0)\right)\left(1+Q\left(X_{N}(0)\right)\right)\left(\lambda_{1}-\lambda_{2}\right) .
\end{aligned}
$$

Let $T(\delta)=\inf \left\{t \geq 0:\left|M_{N}(t)\right|>\delta\right\}$, and denote $Q\left(X_{N}(0)\right)=q_{0}$. Then a standard exponential martingale argument, using the bound on the quantity $R_{N}\left(X_{N}(t)\right)$ above, shows that, given $t_{0}>0, N$ large enough and $0 \leq \delta \leq$ $16 \log 2 t_{0} q_{0}\left(1+q_{0}\right)$,

$$
\mathbb{P}\left(T(\delta) \leq t_{0} \wedge T_{1} \wedge T_{2}\right) \leq 2 e^{-\delta^{2} / 32 t_{0} q_{0}\left(1+q_{0}\right)\left(\lambda_{1}-\lambda_{2}\right)} .
$$

Also, by Grönwall's inequality, on the event $t_{0}<T_{1} \wedge T(\delta)$,

$$
\sup _{t \leq t_{0}} Q_{N}\left(X_{N}(t)\right) \leq\left(Q_{N}\left(X_{N}(0)\right)+\sup _{t \leq t_{0}}\left|M_{N}(t)\right|\right) e^{3\left(\lambda_{1}-\lambda_{2}\right) t_{0}} \leq\left(q_{0}+\delta\right) e^{3\left(\lambda_{1}-\lambda_{2}\right) t_{0}} .
$$

Furthermore, on the event $t_{0}<T_{1} \wedge T(\delta)$, $\inf _{t \leq t_{0}} Q_{N}\left(X_{N}(t)\right) \geq q_{0}-\delta$. In other words, on the event $t_{0}<T_{1} \wedge T(\delta)$,

$$
\sup _{t \leq t_{0}}\left|Q_{N}\left(X_{N}(t)\right)-q_{0}\right| \leq \delta e^{3\left(\lambda_{1}-\lambda_{2}\right) t_{0}}+q_{0}\left(e^{3\left(\lambda_{1}-\lambda_{2}\right) t_{0}}-1\right) .
$$

Let $\psi=\psi(N) \rightarrow \infty$ as $N \rightarrow \infty$, in such a way that $\psi\left(\lambda_{1}-\lambda_{2}\right) \rightarrow 0$, and let $t_{0}(N)=\left(\lambda_{1}-\lambda_{2}\right)^{-1} \psi(N)^{-1}$. We also let $\delta=\psi_{N}^{-1 / 4}$, so $\delta \leq 16 t_{0} \log 2 q_{0}(1+$ 
EXTINCTION TIME FOR THE WEAKER OF TWO COMPETING SIS EPIDEMICS 37

$q_{0}$ ) for $N$ sufficiently large. It follows that, for $N$ sufficiently large, on the event $t_{0}<T_{1} \wedge T\left(\psi(N)^{-1 / 4}\right)$,

$$
\sup _{t \leq t_{0}}\left|Q_{N}\left(X_{N}(t)\right)-q_{0}\right| \leq 2 \psi(N)^{-1 / 4} .
$$

Let $T_{3}=\inf \left\{t \geq 0: \mid Q_{N}\left(X_{N}(t)-q_{0} \mid>2 \psi(N)^{-1 / 4}\right\}\right.$. Clearly, $\mathbb{P}\left(T_{2}<T_{3}\right)=$ 0 . Then we have shown that, with $t_{0}=\left(\lambda_{1}-\lambda_{2}\right)^{-1} \psi(N)^{-1}$ as above,

$$
\begin{aligned}
\mathbb{P}\left(T_{3} \leq t_{0}\right) & \leq \mathbb{P}\left(T_{1} \wedge T\left(\psi(N)^{-1 / 4}\right) \leq t_{0} \wedge T_{3}\right) \\
& \leq \mathbb{P}\left(T\left(\psi(N)^{-1 / 4}\right) \leq t_{0} \wedge T_{1} \wedge T_{3}\right)+\mathbb{P}\left(T_{1} \leq t_{0} \wedge T_{3}\right) \\
& \leq 2 e^{-\psi(N)^{1 / 2} / 32 q_{0}\left(1+q_{0}\right)}+\mathbb{P}\left(T_{1} \leq t_{0} \wedge T_{3}\right) .
\end{aligned}
$$

Let $T_{4}=\inf \left\{t \geq 0: X_{N, 1}(t)+X_{N, 2}(t)<N\left(\lambda_{1}-1\right) / 4\right\}$. Then, if $N$ is sufficiently large, $\mathbb{P}\left(T_{1} \leq t_{0} \wedge T_{3}\right) \leq \mathbb{P}\left(T_{4} \leq t_{0}\right)$. We will use Lemma 10, and Lemma 11, with $\lambda=\lambda_{2}, \mu=1, \omega=8 \sqrt{N\left(\lambda_{2}-1\right)^{2}}$. Note $\left(\lambda_{2}-\right.$ $1)^{-1} e^{\sqrt{N\left(\lambda_{2}-1\right)^{2}}} \geq\left(\lambda_{1}-\lambda_{2}\right)^{-1} \geq t_{0}$ for large $N$, since

$$
e^{\sqrt{N\left(\lambda_{2}-1\right)^{2}}} \geq e^{\left(\frac{N\left(\lambda_{2}-1\right)^{2}}{N\left(\lambda_{1}-\lambda_{2}\right)^{2}}\right)^{1 / 2}} \geq\left(\frac{N\left(\lambda_{2}-1\right)^{2}}{N\left(\lambda_{1}-\lambda_{2}\right)^{2}}\right)^{1 / 2}=\frac{\lambda_{2}-1}{\lambda_{1}-\lambda_{2}} .
$$

Hence $\mathbb{P}\left(T_{4} \leq t_{0}\right) \leq 4 e^{-\sqrt{N}\left(\lambda_{2}-1\right)}$, and the result follows, as $q_{0} \leq 2 \alpha / \beta$ for large $N$.

For the next phase, after time $\left(\lambda_{1}-\lambda_{2}\right)^{-1} \psi^{-1}$, we approximate vector $\tilde{x}_{N}(t)$ by the solution $\tilde{x}(t)$ to (9). When $\mu_{1}=\mu_{2}=1$, then equation (9) takes the form:

$$
\begin{aligned}
\frac{d \tilde{x}_{1}(t)}{d t} & =-\left(\lambda_{1}-1\right) \tilde{x}_{1}(t)-\lambda_{1} \tilde{x}_{1}(t)^{2}-\frac{\left(\lambda_{1}-\lambda_{2}\right)^{2}}{\lambda_{1}\left(\lambda_{1}-1\right)}\left(\frac{\tilde{x}_{2}(t)}{a}\right)^{2}+\frac{\left(\lambda_{1}-\lambda_{2}\right) \lambda_{1}}{\lambda_{1}-1} \tilde{x}_{1}(t) \frac{\tilde{x}_{2}(t)}{a} \\
\frac{d \tilde{x}_{2}(t)}{d t} & =-\frac{\lambda_{1}-\lambda_{2}}{\lambda_{1}} \tilde{x}_{2}(t)-\lambda_{2} \tilde{x}_{2}(t) \tilde{x}_{1}(t)+\frac{\lambda_{2}}{\lambda_{1} a} \frac{\lambda_{1}-\lambda_{2}}{\lambda_{1}-1} \tilde{x}_{2}(t)^{2} .
\end{aligned}
$$

Lemma 14. Assume that $X_{N, 1}(0)+X_{N, 2}(0) \leq 2 N\left(\lambda_{1}-1\right) / \lambda_{1}, X_{N, 1}(0) / X_{N, 2}(0)=$ $\alpha / \beta+\varepsilon_{N}$, where $\varepsilon_{N} \rightarrow 0$ as $N \rightarrow \infty$. Let $x_{i}(0)=N^{-1} X_{N, i}(0)$ for $i=1,2$, with $\tilde{x}_{i}(0)$ being derived from $x_{i}(0)$ according to the change of variables. Let $\omega=\omega(N)>0$, where $\omega(N) \rightarrow \infty$ with $N$ and $N\left(\lambda_{1}-\lambda_{2}\right)^{2} / \omega(N) \rightarrow \infty$. For $t \geq 0$, let

$$
f_{N}(t)=\max \left\{\frac{\lambda_{1}-1}{\lambda_{1}-\lambda_{2}}\left|\tilde{x}_{N, 1}(t)-\tilde{x}_{1}(t)\right|,\left|\tilde{x}_{N, 2}(t)-\tilde{x}_{2}(t)\right|\right\} .
$$

Then, for $N$ large enough,

$$
\mathbb{P}\left(\sup _{t \leq\left(\lambda_{1}-1\right)^{-1} e^{\omega / 8}} f_{N}(t)>8 \frac{\lambda_{1}-1}{\lambda_{1}-\lambda_{2}} \sqrt{\frac{\omega}{N}} e^{32\left(\lambda_{1} \frac{\beta}{\alpha}+1\right)}\right) \leq 12 e^{-\omega / 8} .
$$


Proof. Using the integral form of (9) and its stochastic analogue as in Section 4, writing $\eta_{1}=\lambda_{1}-1$ and $\eta_{2}=\left(\lambda_{1}-\lambda_{2}\right) / \lambda_{1}$ and noting that $\eta_{1}>\eta_{2}$ for large $N$, we can write

$$
\begin{aligned}
\left|\tilde{x}_{N, 1}(t)-\tilde{x}_{1}(t)\right| & \leq\left|\tilde{x}_{N, 1}(0)-\tilde{x}_{1}(0)\right| e^{-t \eta_{1}}+\left|\int_{0}^{t} e^{-\eta_{1}(t-s)} d M_{N, 1}(s)\right| \\
& +\lambda_{1} \int_{0}^{t} e^{-\eta_{1}(t-s)}\left|\tilde{x}_{N, 1}(s)-\tilde{x}_{1}(s)\right|\left|\tilde{x}_{N, 1}(s)+\tilde{x}_{1}(s)\right| d s \\
& +\frac{\left(\lambda_{1}-\lambda_{2}\right)^{2}}{\lambda_{1}\left(\lambda_{1}-1\right) a^{2}} \int_{0}^{t} e^{-\eta_{1}(t-s)}\left|\tilde{x}_{N, 2}(s)-\tilde{x}_{2}(s)\right|\left(\tilde{x}_{N, 2}(s)+\tilde{x}_{2}(s)\right) d s \\
& +\frac{\left(\lambda_{1}-\lambda_{2}\right) \lambda_{1}}{a\left(\lambda_{1}-1\right)} \int_{0}^{t} e^{-\eta_{1}(t-s)}\left|\tilde{x}_{N, 1}(s)-\tilde{x}_{1}(s)\right| \tilde{x}_{N, 2}(s) d s \\
& +\frac{\left(\lambda_{1}-\lambda_{2}\right) \lambda_{1}}{a\left(\lambda_{1}-1\right)} \int_{0}^{t} e^{-\eta_{1}(t-s)}\left|\tilde{x}_{N, 2}(s)-\tilde{x}_{2}(s)\right|\left|\tilde{x}_{1}(s)\right| d s,
\end{aligned}
$$

and

$$
\begin{aligned}
& \left|\tilde{x}_{N, 2}(t)-\tilde{x}_{2}(t)\right| \\
& \quad \leq\left|\tilde{x}_{N, 2}(0)-\tilde{x}_{2}(0)\right| e^{-t \eta_{2}}+\lambda_{2} \int_{0}^{t} e^{-(t-s) \eta_{2}}\left|\tilde{x}_{N, 2}(s)-\tilde{x}_{2}(s)\right|\left|\tilde{x}_{N, 1}(s)\right| d s \\
& \quad+\lambda_{2} \int_{0}^{t} e^{-(t-s) \eta_{2}}\left|\tilde{x}_{N, 1}(s)-\tilde{x}_{1}(s)\right| \tilde{x}_{2}(s) d s+\left|\int_{0}^{t} e^{-(t-s) \eta_{2}} d M_{N, 2}(s)\right| \\
& \quad+\frac{\lambda_{2}}{\lambda_{1} a} \frac{\lambda_{1}-\lambda_{2}}{\lambda_{1}-1} \int_{0}^{t} e^{-(t-s) \eta_{2}}\left|\tilde{x}_{N, 2}(s)-\tilde{x}_{2}(s)\right|\left(\tilde{x}_{N, 2}(s)+\tilde{x}_{2}(s)\right) d s
\end{aligned}
$$

Let

$$
f_{N}(t)=\max \left\{\left|\tilde{x}_{N, 1}(t)-\tilde{x}_{1}(t)\right| \frac{\lambda_{1}-1}{\lambda_{1}-\lambda_{2}},\left|\tilde{x}_{N, 2}(t)-\tilde{x}_{2}(t)\right|\right\},
$$

and let $g_{N}(t)=e^{t \eta_{2}} f_{N}(t)$. Then

$$
\begin{aligned}
& \frac{\lambda_{1}-1}{\lambda_{1}-\lambda_{2}}\left|\tilde{x}_{N, 1}(t)-\tilde{x}_{1}(t)\right| e^{t \eta_{2}} \\
& \leq \quad g_{N}(0)+\lambda_{1} \int_{0}^{t} g_{N}(s)\left|\tilde{x}_{N, 1}(s)+\tilde{x}_{1}(s)\right| d s \\
& \quad+\frac{\lambda_{1}-\lambda_{2}}{\lambda_{1} a^{2}} \int_{0}^{t} g_{N}(s)\left(\tilde{x}_{N, 2}(s)+\tilde{x}_{2}(s)\right) d s+\frac{\left(\lambda_{1}-\lambda_{2}\right) \lambda_{1}}{a\left(\lambda_{1}-1\right)} \int_{0}^{t} g_{N}(s) \tilde{x}_{N, 2}(s) d s \\
& \quad+\frac{\lambda_{1}}{a} \int_{0}^{t} g_{N}(s)\left|\tilde{x}_{1}(s)\right| d s+\frac{\lambda_{1}-1}{\lambda_{1}-\lambda_{2}} e^{t \eta_{2}}\left|\int_{0}^{t} e^{-\eta_{1}(t-s)} d M_{N, 1}(s)\right|
\end{aligned}
$$


EXTINCTION TIME FOR THE WEAKER OF TWO COMPETING SIS EPIDEMICS 39

and

$$
\begin{aligned}
& \left|\tilde{x}_{N, 2}(t)-\tilde{x}_{2}(t)\right| e^{t \eta_{2}} \\
& \quad \leq \quad g_{N}(0)+\lambda_{2} \int_{0}^{t} g_{N}(s)\left|\tilde{x}_{N, 1}(s)\right| d s+\lambda_{2} \frac{\lambda_{1}-\lambda_{2}}{\lambda_{1}-1} \int_{0}^{t} g_{N}(s) \tilde{x}_{2}(s) d s \\
& \quad+\frac{\lambda_{2}}{\lambda_{1} a} \frac{\lambda_{1}-\lambda_{2}}{\lambda_{1}-1} \int_{0}^{t} g_{N}(s)\left(\tilde{x}_{N, 2}(s)+\tilde{x}_{2}(s)\right) d s+e^{t \eta_{2}}\left|\int_{0}^{t} e^{-(t-s) \eta_{2}} d M_{N, 2}(s)\right| .
\end{aligned}
$$

It follows that

$$
\begin{aligned}
& \frac{\lambda_{1}-1}{\lambda_{1}-\lambda_{2}}\left|\tilde{x}_{N, 1}(t)-\tilde{x}_{1}(t)\right| e^{t \eta_{2}} \\
& \leq g_{N}(0)+2 \lambda_{1} \int_{0}^{t} g_{N}(s)\left|\tilde{x}_{1}(s)\right| d s+\frac{\lambda_{1}\left(\lambda_{1}-\lambda_{2}\right)}{\lambda_{1}-1} \int_{0}^{t} g_{N}(s)^{2} e^{-s \eta_{2}} d s \\
& \quad+\frac{2\left(\lambda_{1}-\lambda_{2}\right)}{\lambda_{1} a^{2}} \int_{0}^{t} g_{N}(s) \tilde{x}_{2}(s) d s+\frac{\lambda_{1}-\lambda_{2}}{\lambda_{1} a^{2}} \int_{0}^{t} g_{N}(s)^{2} e^{-s \eta_{2}} d s \\
& +\frac{\left(\lambda_{1}-\lambda_{2}\right) \lambda_{1}}{a\left(\lambda_{1}-1\right)} \int_{0}^{t} g_{N}(s) \tilde{x}_{2}(s) d s+\frac{\left(\lambda_{1}-\lambda_{2}\right) \lambda_{1}}{a\left(\lambda_{1}-1\right)} \int_{0}^{t} g_{N}(s)^{2} e^{-s \eta_{2}} d s \\
& +\frac{\lambda_{1}}{a} \int_{0}^{t} g_{N}(s)\left|\tilde{x}_{1}(s)\right| d s+\frac{\lambda_{1}-1}{\lambda_{1}-\lambda_{2}} e^{t \eta_{2}}\left|\int_{0}^{t} e^{-\eta_{1}(t-s)} d M_{N, 1}(s)\right|,
\end{aligned}
$$

and

$$
\begin{aligned}
& \left|\tilde{x}_{N, 2}(t)-\tilde{x}_{2}(t)\right| e^{t \eta_{2}} \\
& \quad \leq \quad g_{N}(0)+\lambda_{2} \int_{0}^{t} g_{N}(s)\left|\tilde{x}_{1}(s)\right| d s+\lambda_{2} \frac{\lambda_{1}-\lambda_{2}}{\lambda_{1}-1} \int_{0}^{t} g_{N}(s)^{2} e^{-s \eta_{2}} d s \\
& \quad+\lambda_{2} \frac{\lambda_{1}-\lambda_{2}}{\lambda_{1}-1} \int_{0}^{t} g_{N}(s) \tilde{x}_{2}(s) d s+\frac{2 \lambda_{2}\left(\lambda_{1}-\lambda_{2}\right)}{a \lambda_{1}\left(\lambda_{1}-1\right)} \int_{0}^{t} g_{N}(s) \tilde{x}_{2}(s) d s \\
& \quad+\frac{\lambda_{2}}{\lambda_{1} a} \frac{\lambda_{1}-\lambda_{2}}{\lambda_{1}-1} \int_{0}^{t} g_{N}(s)^{2} e^{-s \eta_{2}} d s+e^{t \eta_{2}}\left|\int_{0}^{t} e^{-(t-s) \eta_{2}} d M_{N, 2}(s)\right| .
\end{aligned}
$$

So, since $a \rightarrow 1$ as $N \rightarrow \infty$, for $N$ large enough,

$$
\begin{aligned}
& \frac{\lambda_{1}-1}{\lambda_{1}-\lambda_{2}}\left|\tilde{x}_{N, 1}(t)-\tilde{x}_{1}(t)\right| e^{t \eta_{2}} \\
& \quad \leq \quad g_{N}(0)+4 \lambda_{1} \int_{0}^{t} g_{N}(s)\left|\tilde{x}_{1}(s)\right| d s+\frac{2 \lambda_{1}\left(\lambda_{1}-\lambda_{2}\right)}{\lambda_{1}-1} \int_{0}^{t} g_{N}(s) \tilde{x}_{2}(s) d s \\
& \quad+2 \frac{\lambda_{1}\left(\lambda_{1}-\lambda_{2}\right)}{\lambda_{1}-1} \int_{0}^{t} g_{N}(s)^{2} e^{-s \eta_{2}} d s+\frac{\lambda_{1}-1}{\lambda_{1}-\lambda_{2}} e^{t \eta_{2}}\left|\int_{0}^{t} e^{-\eta_{1}(t-s)} d M_{N, 1}(s)\right|,
\end{aligned}
$$


and

$$
\begin{aligned}
& \left|\tilde{x}_{N, 2}(t)-\tilde{x}_{2}(t)\right| e^{t \eta_{2}} \\
& \quad \leq \quad g_{N}(0)+\lambda_{2} \int_{0}^{t} g_{N}(s)\left|\tilde{x}_{1}(s)\right| d s+4 \frac{\lambda_{2}\left(\lambda_{1}-\lambda_{2}\right)}{\lambda_{1}-1} \int_{0}^{t} g_{N}(s) \tilde{x}_{2}(s) d s \\
& \quad+4 \frac{\lambda_{2}\left(\lambda_{1}-\lambda_{2}\right)}{\lambda_{1}-1} \int_{0}^{t} g_{N}(s)^{2} e^{-s \eta_{2}} d s+e^{t \eta_{2}}\left|\int_{0}^{t} e^{-(t-s) \eta_{2}} d M_{N, 2}(s)\right| .
\end{aligned}
$$

Hence, if $N$ is large enough,

$$
\begin{aligned}
g_{N}(t) & \leq g_{N}(0)+4 \lambda_{1} \int_{0}^{t} g_{N}(s)\left|\tilde{x}_{1}(s)\right| d s+4 \frac{\lambda_{1}\left(\lambda_{1}-\lambda_{2}\right)}{\lambda_{1}-1} \int_{0}^{t} g_{N}(s) \tilde{x}_{2}(s) d s \\
& +4 \frac{\lambda_{1}\left(\lambda_{1}-\lambda_{2}\right)}{\lambda_{1}-1} \int_{0}^{t} g_{N}(s)^{2} e^{-s \eta_{2}} d s+e^{t \eta_{2}} M
\end{aligned}
$$

where $M$ is the maximum of $\frac{\lambda_{1}-1}{\lambda_{1}-\lambda_{2}}\left|\int_{0}^{t} e^{-\eta_{1}(t-s)} d M_{N, 1}(s)\right|$ and $\left|\int_{0}^{t} e^{-(t-s) \eta_{2}} d M_{N, 2}(s)\right|$.

Let $T_{1}$ be the infimum of times $t$ such that $x_{N, 1}(t)+x_{N, 2}(t)>4\left(\lambda_{1}-1\right) / \lambda_{1}$. Similarly to the calculations in the proof of Lemma 6, on the event $t<T_{1}$, for $N$ large enough,

$$
\begin{aligned}
\int_{0}^{t} \sum_{y} q\left(\tilde{x}_{N}(s), \tilde{x}_{N}(s)+y\right)\left(e^{\tilde{A}(t-s)} y\right)_{1}^{2} d s & \leq \frac{4\left(\lambda_{1}+1\right)}{N} \int_{0}^{t} \frac{\lambda_{1}-1}{\lambda_{1}} e^{-2(t-s) \eta_{1}} d s \\
& \leq \frac{2\left(\lambda_{1}+1\right)}{\lambda_{1} N} \leq \frac{4}{N}
\end{aligned}
$$

and

$$
\begin{aligned}
& \int_{0}^{t} \sum_{y} q\left(\tilde{x}_{N}(s), \tilde{x}_{N}(s)+y\right)\left(e^{\tilde{A}(t-s)} y\right)_{2}^{2} d s \\
& \quad \leq \frac{4\left(\lambda_{1}+1\right)\left(\lambda_{1}-1\right)}{N\left(\lambda_{1}-\lambda_{2}\right)} \int_{0}^{t} \frac{\lambda_{1}-\lambda_{2}}{\lambda_{1}} e^{-2(t-s) \eta_{2}} d s \leq \frac{4 \lambda_{1}\left(\lambda_{1}-1\right)}{N\left(\lambda_{1}-\lambda_{2}\right)} .
\end{aligned}
$$

Given $\omega=\omega(N)$ as in the statement of the lemma, let $T_{2}$ be the infimum of times $t$ such that

$$
\left|\int_{0}^{t} e^{-\left(\lambda_{1}-1\right)(t-s)} d M_{N, 1}(s)\right|>6 \sqrt{\frac{\omega}{N}}
$$

or

$$
\left|\int_{0}^{t} e^{-(t-s) \frac{\lambda_{1}-\lambda_{2}}{\lambda_{1}}} d M_{N, 2}(s)\right|>6 \sqrt{\frac{\omega \lambda_{1}\left(\lambda_{1}-1\right)}{N\left(\lambda_{1}-\lambda_{2}\right)}} .
$$

Then on the event $t<T_{1} \wedge T_{2}$, for $N$ large enough,

$$
\begin{aligned}
g_{N}(t) & \leq g_{N}(0)+4 \lambda_{1} \int_{0}^{t} g_{N}(s)\left|\tilde{x}_{1}(s)\right| d s+\frac{4 \lambda_{1}\left(\lambda_{1}-\lambda_{2}\right)}{\lambda_{1}-1} \int_{0}^{t} g_{N}(s) \tilde{x}_{2}(s) d s \\
& +\frac{4 \lambda_{1}\left(\lambda_{1}-\lambda_{2}\right)}{\lambda_{1}-1} \int_{0}^{t} g_{N}(s)^{2} e^{-s \eta_{2}} d s+6 e^{t \eta_{2}} \frac{\lambda_{1}-1}{\lambda_{1}-\lambda_{2}} \sqrt{\frac{\omega}{N}}
\end{aligned}
$$


EXTINCTION TIME FOR THE WEAKER OF TWO COMPETING SIS EPIDEMICS 41

Let $T_{3}$ be the infimum of times $t$ such that

$$
g_{N}(t)>10 e^{32\left(\lambda_{1} \beta / \alpha+1\right)} e^{t \eta_{2}} \frac{\lambda_{1}-1}{\lambda_{1}-\lambda_{2}} \sqrt{\frac{\omega}{N}} .
$$

On the event $t<T_{1} \wedge T_{2} \wedge T_{3}$, for $N$ large enough,

$$
\begin{aligned}
\frac{4 \lambda_{1}\left(\lambda_{1}-\lambda_{2}\right)}{\lambda_{1}-1} \int_{0}^{t} g_{N}(s)^{2} e^{-s \eta_{2}} d s & \leq 400 \lambda_{1}^{2} e^{64\left(\lambda_{1} \beta / \alpha+1\right)} \frac{\left(\lambda_{1}-1\right)}{\left(\lambda_{1}-\lambda_{2}\right)^{2}} \frac{\omega}{N} e^{t \eta_{2}} \\
& \leq e^{t \eta_{2}} \frac{\lambda_{1}-1}{\lambda_{1}-\lambda_{2}} \sqrt{\frac{\omega}{N}}
\end{aligned}
$$

since we have assumed that $N\left(\lambda_{1}-\lambda_{2}\right)^{2} / \omega \rightarrow \infty$. It follows that, for $N$ large enough, on the event $t<T_{1} \wedge T_{2} \wedge T_{3}$,

$$
\begin{aligned}
g_{N}(t) \leq & g_{N}(0)+4 \lambda_{1} \int_{0}^{t} g_{N}(s)\left|\tilde{x}_{1}(s)\right| d s+\frac{4 \lambda_{1}\left(\lambda_{1}-\lambda_{2}\right)}{\lambda_{1}-1} \int_{0}^{t} g_{N}(s) \tilde{x}_{2}(s) d s \\
& +7 e^{t \eta_{2}} \frac{\lambda_{1}-1}{\lambda_{1}-\lambda_{2}} \sqrt{\frac{\omega}{N}} \\
\leq & 8 e^{t \eta_{2}} \frac{\lambda_{1}-1}{\lambda_{1}-\lambda_{2}} \sqrt{\frac{\omega}{N}}+4 \lambda_{1} \int_{0}^{t} g_{N}(s)\left|\tilde{x}_{1}(s)\right| d s \\
& +\frac{4 \lambda_{1}\left(\lambda_{1}-\lambda_{2}\right)}{\lambda_{1}-1} \int_{0}^{t} g_{N}(s) \tilde{x}_{2}(s) d s,
\end{aligned}
$$

provided $g_{N}(0)=f_{N}(0) \leq \frac{\lambda_{1}-1}{\lambda_{1}-\lambda_{2}} \sqrt{\frac{\omega}{N}}$. By Grönwall's lemma, on the event $t<T_{1} \wedge T_{2} \wedge T_{3}$, for $N$ large enough,

$$
g_{N}(t) \leq 8 e^{t \eta_{2}} \frac{\lambda_{1}-1}{\lambda_{1}-\lambda_{2}} \sqrt{\frac{\omega}{N}} e^{4 \lambda_{1} \int_{0}^{t}\left|\tilde{x}_{1}(s)\right| d s+\frac{4 \lambda_{1}\left(\lambda_{1}-\lambda_{2}\right)}{\lambda_{1}-1} \int_{0}^{t} \tilde{x}_{2}(s) d s} .
$$

Now, by (5),

$$
\begin{aligned}
\tilde{x}_{2}(t) & =x_{2}(t)=\left(x_{1}(t) / x_{1}(0)\right)^{\lambda_{2} / \lambda_{1}} x_{2}(0) e^{-t \frac{\lambda_{1}-\lambda_{2}}{\lambda_{1}}} \\
& \leq\left(x_{2}(0) / x_{1}(0)\right)^{\lambda_{2} / \lambda_{1}} x_{1}(t)^{\lambda_{2} / \lambda_{1}} e^{-t \frac{\lambda_{1}-\lambda_{2}}{\lambda_{1}}} \\
& =\left(\frac{\beta}{\alpha}(1+o(1))\right)^{\lambda_{2} / \lambda_{1}} x_{1}(t)^{\lambda_{2} / \lambda_{1}} e^{-t \frac{\lambda_{1}-\lambda_{2}}{\lambda_{1}}} \leq 4(\beta / \alpha) \frac{\lambda_{1}-1}{\lambda_{1}} e^{-t \frac{\lambda_{1}-\lambda_{2}}{\lambda_{1}}}
\end{aligned}
$$

where we have also used the facts that $x_{1}(0)+x_{2}(0) \leq 2\left(\lambda_{1}-1\right) / \lambda_{1}$ implies $x_{1}(t)+x_{2}(t) \leq 2\left(\lambda_{1}-1\right) / \lambda_{1}$ for all $t$, and that $\left(\lambda_{1}-\lambda_{2}\right) \log \left(\lambda_{1}-1\right) \rightarrow 0$ (and so $x_{1}(t)^{\lambda_{2} / \lambda_{1}} \leq 3\left(\lambda_{1}-1\right) / \lambda_{1}$ for $N$ large enough). Thus

$$
\frac{4 \lambda_{1}\left(\lambda_{1}-\lambda_{2}\right)}{\lambda_{1}-1} \int_{0}^{t} \tilde{x}_{2}(s) d s \leq 16 \lambda_{1}(\beta / \alpha) .
$$

Also, while $\tilde{x}_{1}(t) \geq 0$, using (9), we have for $N$ large enough,

$$
\frac{d \tilde{x}_{1}(t)}{d t} \leq-\tilde{x}_{1}(t)\left(\left(\lambda_{1}-1\right)-\frac{\left(\lambda_{1}-\lambda_{2}\right) \lambda_{1}}{\left(\lambda_{1}-1\right) a} \tilde{x}_{2}(t)\right) \leq-\frac{\lambda_{1}-1}{2} \tilde{x}_{1}(t),
$$


since $\tilde{x}_{2}(t)=x_{2}(t) \leq 2\left(\lambda_{1}-1\right) / \lambda_{1}$ for all $t$. On the other hand, when $\tilde{x}_{1}(t)$ becomes negative, and while $\left|\tilde{x}_{1}(t)\right| \leq\left(\lambda_{1}-1\right) / 4 \lambda_{1}$, then for $N$ large enough,

$$
\frac{d \tilde{x}_{1}(t)}{d t} \geq-\frac{\lambda_{1}-1}{2} \tilde{x}_{1}(t)-\frac{\left(\lambda_{1}-\lambda_{2}\right)^{2}}{\lambda_{1}\left(\lambda_{1}-1\right)}\left(\frac{\tilde{x}_{2}(t)}{a}\right)^{2},
$$

and so, using $x_{2}(t) \leq 4(\beta / \alpha) \frac{\lambda_{1}-1}{\lambda_{1}} e^{-t\left(\lambda_{1}-\lambda_{2}\right) / \lambda_{1}}$,

$$
\tilde{x}_{1}(t) \geq-\frac{32 \beta^{2}}{\alpha^{2} a^{2} \lambda_{1}^{2}}\left(\lambda_{1}-\lambda_{2}\right)^{2} e^{-2 t\left(\lambda_{1}-\lambda_{2}\right) / \lambda_{1}} .
$$

Since $\tilde{x}_{1}(0) \leq 4\left(\lambda_{1}-1\right) / \lambda_{1}$ for $N$ large enough, we see that, if $N$ is large enough, then $4 \lambda_{1} \int_{0}^{t}\left|\tilde{x}_{1}(s)\right| d s \leq 32$.

It follows that if $N$ is large enough, then on the event $t<T_{1} \wedge T_{2} \wedge T_{3}$,

$$
g_{N}(t) \leq 8 e^{t\left(\lambda_{1}-\lambda_{2}\right) / \lambda_{1}} \frac{\lambda_{1}-1}{\lambda_{2}-\lambda_{1}} \sqrt{\frac{\omega}{N}} e^{32\left(\lambda_{1} \beta / \alpha+1\right)} .
$$

and so

$$
f_{N}(t) \leq 8 \frac{\lambda_{1}-1}{\lambda_{1}-\lambda_{2}} \sqrt{\frac{\omega}{N}} e^{32\left(\lambda_{1} \beta / \alpha+1\right)} .
$$

Let $T_{4}=\inf \left\{t: f_{N}(t)>8 \frac{\lambda_{1}-1}{\lambda_{2}-\lambda_{1}} \sqrt{\frac{\omega}{N}} e^{32\left(\lambda_{1} \beta / \alpha+1\right)}\right\}$. Let $t_{0}=t_{0}(N)=$ $\left(\lambda_{1}-1\right)^{-1} e^{\omega / 8}$. By the above,

$\mathbb{P}\left(T_{4} \leq t_{0}\right) \leq \mathbb{P}\left(T_{1} \wedge T_{2} \wedge T_{3} \leq T_{4} \wedge t_{0}\right) \leq \mathbb{P}\left(T_{1} \leq t_{0}\right)+\mathbb{P}\left(T_{2} \leq T_{1} \wedge t_{0}\right)+\mathbb{P}\left(T_{3} \leq T_{4}\right)$.

By Lemma 10, and by Lemma 11 with $\lambda=\lambda_{1}$ and $\mu=1$, if $N$ is large enough, then $\mathbb{P}\left(T_{1} \leq t_{0}\right) \leq 4 e^{-\omega / 8}$. By Lemma 7 applied to $\left(\tilde{x}_{N}(t)\right)$, this time taking $\sigma=\left(\lambda_{1}-1\right)^{-1}, K_{1}=4 / N, K_{2}=4 \lambda_{1}\left(\lambda_{1}-1\right) / N\left(\lambda_{1}-\lambda_{2}\right)$, $\mathbb{P}\left(T_{2} \leq T_{1} \wedge t_{0}\right) \leq 8 e^{-\omega / 8}$. Also, clearly, for $N$ large enough, $\mathbb{P}\left(T_{3} \leq T_{4}\right)=0$. It follows that $\mathbb{P}\left(T_{4} \leq t_{0}\right) \leq 12 e^{-\omega / 8}$, as required.

Lemma 15. Let $\omega_{1}=\omega_{1}(N) \rightarrow \infty$ as $N \rightarrow \infty$. Let the assumptions of Lemma 14 on $X_{N}(0)$ and $x(0)$ be satisfied. Assume further that

$$
N\left(\lambda_{1}-\lambda_{2}\right)^{2} / \log \left(\frac{\lambda_{1}-1}{\lambda_{1}-\lambda_{2}}\right) e^{\omega_{1} / 2} \rightarrow \infty .
$$

For $t \geq 0$, let $f_{N}(t)=\left|\tilde{x}_{N, 2}(t)-\tilde{x}_{2}(t)\right|$. Set

$$
\delta_{N}(t)=\left(2\left(\lambda_{1}-\lambda_{2}\right)^{-1} \log ^{1 / 2}\left(\frac{\lambda_{1}-1}{\lambda_{1}-\lambda_{2}}\right) e^{\omega_{1} / 8} x_{2}(t)+8 \sqrt{\frac{\lambda_{1}\left(\lambda_{1}-1\right)}{\lambda_{1}-\lambda_{2}}}\right) \sqrt{\frac{\omega_{1}}{N}} e^{32(1+\beta / \alpha)} .
$$

Then, for $N$ large enough,

$$
\mathbb{P}\left(\sup _{t \leq \frac{\lambda_{1}}{\lambda_{1}-\lambda_{2}} e^{\omega_{1} / 8}} f_{N}(t)>\delta_{N}(t)\right) \leq 16 e^{-\omega_{1} / 8} .
$$

Proof. Let $\omega_{2}=\log \left(\frac{\lambda_{1}\left(\lambda_{1}-1\right)}{\lambda_{1}-\lambda_{2}}\right)+\omega_{1}$, and note that $N\left(\lambda_{1}-\lambda_{2}\right)^{2} / \omega_{2} \rightarrow \infty$. 
EXTINCTION TIME FOR THE WEAKER OF TWO COMPETING SIS EPIDEMICS 43

As in the proof of Lemma 14, with $\eta_{2}=\left(\lambda_{1}-\lambda_{2}\right) / \lambda_{1}$,

$$
\begin{aligned}
& f_{N}(t)=\left|\tilde{x}_{N, 2}(t)-\tilde{x}_{2}(t)\right| \\
& \quad \leq\left|\tilde{x}_{N, 2}(0)-\tilde{x}_{2}(0)\right| e^{-t \eta_{2}}+\lambda_{2} \int_{0}^{t} e^{-(t-s) \eta_{2}}\left|\tilde{x}_{N, 2}(s)-\tilde{x}_{2}(s)\right|\left|\tilde{x}_{N, 1}(s)\right| d s \\
& \quad+\lambda_{2} \int_{0}^{t} e^{-(t-s) \eta_{2}}\left|\tilde{x}_{N, 1}(s)-\tilde{x}_{1}(s)\right| \tilde{x}_{2}(s) d s+\left|\int_{0}^{t} e^{-(t-s) \eta_{2}} d M_{N, 2}(s)\right| \\
& \quad+\frac{\lambda_{2}}{\lambda_{1} a} \frac{\lambda_{1}-\lambda_{2}}{\lambda_{1}-1} \int_{0}^{t} e^{-(t-s) \eta_{2}}\left|\tilde{x}_{N, 2}(s)-\tilde{x}_{2}(s)\right|\left(\tilde{x}_{N, 2}(s)+\tilde{x}_{2}(s)\right) d s
\end{aligned}
$$

Let $g_{N}(t)=f_{N}(t) e^{t \eta_{2}}$. Let $T_{1}$ be the infimum of times $t$ such that

$$
\left|\tilde{x}_{N, 1}(t)-\tilde{x}_{1}(t)\right|>8 \sqrt{\frac{\omega_{2}}{N}} e^{32\left(\lambda_{1} \beta / \alpha+1\right)} .
$$

Let $T_{2}$ be the infimum of times $t$ such that

$$
\left|\int_{0}^{t} e^{-(t-s) \eta_{2}} d M_{N, 2}(s)\right|>6 \sqrt{\frac{\omega_{1} \lambda_{1}\left(\lambda_{1}-1\right)}{N\left(\lambda_{1}-\lambda_{2}\right)}} .
$$

Then for $t<T_{1} \wedge T_{2}$, if $N$ is large enough,

$$
\begin{aligned}
g_{N}(t) & \leq g_{N}(0)+\lambda_{2} \int_{0}^{t} g_{N}(s)\left|\tilde{x}_{N, 1}(s)\right| d s \\
& +8 \lambda_{2} \sqrt{\frac{\omega_{2}}{N}} e^{32\left(\lambda_{1} \beta / \alpha+1\right)} \int_{0}^{t} e^{s \eta_{2}} \tilde{x}_{2}(s) d s+6 e^{t \eta_{2}} \sqrt{\frac{\omega_{1} \lambda_{1}\left(\lambda_{1}-1\right)}{N\left(\lambda_{1}-\lambda_{2}\right)}} \\
& +\frac{4\left(\lambda_{1}-\lambda_{2}\right)}{\lambda_{1}-1} \int_{0}^{t} g_{N}(s) \tilde{x}_{2}(s) d s+\frac{2\left(\lambda_{1}-\lambda_{2}\right)}{\lambda_{1}-1} \int_{0}^{t} g_{N}(s)^{2} e^{-s \eta_{2}} d s .
\end{aligned}
$$

Let $T_{3}$ be the infimum of times $t$ such that

$$
\begin{aligned}
g_{N}(t)> & 10 \sqrt{\frac{\lambda_{1}\left(\lambda_{1}-1\right)}{\lambda_{1}-\lambda_{2}}} \sqrt{\frac{\omega_{1}}{N}} e^{32(1+\beta / \alpha)} e^{t \eta_{2}} \\
& +4 \sqrt{\frac{\omega_{1}}{N\left(\lambda_{1}-\lambda_{2}\right)^{2}}} \log ^{1 / 2}\left(\frac{\lambda_{1}-1}{\lambda_{1}-\lambda_{2}}\right) e^{32(1+\beta / \alpha)} e^{\omega_{1} / 8} x_{2}(t) e^{t \eta_{2}} .
\end{aligned}
$$

Now, by (19),

$$
x_{2}(t) \leq 4(\beta / \alpha) \frac{\lambda_{1}-1}{\lambda_{1}} e^{-t \eta_{2}},
$$

and, by (5) and (19), the fact that, for all $t, x_{1}(t)+x_{2}(t) \geq \frac{1}{2} \frac{\lambda_{1}-1}{\lambda_{1}}$ if $N$ is large enough, and the fact that $x_{1}(t) / x_{2}(t)$ is increasing, also

$$
x_{2}(t) \geq \frac{1}{4} \frac{\beta}{\alpha+\beta} \frac{\lambda_{1}-1}{\lambda_{1}} e^{-t \eta_{2}} .
$$


Then for $t<T_{3}$, if $N$ is large enough,

$$
\begin{aligned}
& \frac{2\left(\lambda_{1}-\lambda_{2}\right)}{\lambda_{1}-1} \int_{0}^{t} g_{N}(s)^{2} e^{-s \eta_{2}} d s \\
& \leq \frac{200 \omega_{1} \lambda_{1}^{2}}{\left(\lambda_{1}-\lambda_{2}\right) N} e^{64(1+\beta / \alpha)} e^{t \eta_{2}}+\frac{1024(\beta / \alpha)^{2}\left(\lambda_{1}-1\right) \omega_{1}}{N\left(\lambda_{1}-\lambda_{2}\right)^{2}} e^{64(1+\beta / \alpha)} \log \left(\frac{\lambda_{1}-1}{\lambda_{1}-\lambda_{2}}\right) e^{\omega_{1} / 4} \\
& \leq e^{t \eta_{2}} \sqrt{\frac{\omega_{1} \lambda_{1}\left(\lambda_{1}-1\right)}{N\left(\lambda_{1}-\lambda_{2}\right)}}+\sqrt{\frac{\omega_{1}}{N\left(\lambda_{1}-\lambda_{2}\right)^{2}}} \log ^{1 / 2}\left(\frac{\lambda_{1}-1}{\lambda_{1}-\lambda_{2}}\right) e^{\omega_{1} / 8} x_{2}(t) e^{t \eta_{2}},
\end{aligned}
$$

since our assumptions imply that $N\left(\lambda_{1}-1\right)\left(\lambda_{1}-\lambda_{2}\right) / \omega_{1} \rightarrow \infty$ and $N\left(\lambda_{1}-\right.$ $\left.\lambda_{2}\right)^{2} / \log \left(\frac{\lambda_{1}-1}{\lambda_{1}-\lambda_{2}}\right) e^{\omega_{1} / 4} \rightarrow \infty$. So, for $t<T_{1} \wedge T_{2} \wedge T_{3}$, if $N$ is large enough and $g_{N}(0) \leq \sqrt{\frac{\omega_{1} \lambda_{1}\left(\lambda_{1}-1\right)}{N\left(\lambda_{1}-\lambda_{2}\right)}}$,

$$
\begin{aligned}
g_{N}(t) & \leq \lambda_{2} \int_{0}^{t} g_{N}(s)\left|\tilde{x}_{1}(s)\right| d s+\frac{4\left(\lambda_{1}-\lambda_{2}\right)}{\lambda_{1}-1} \int_{0}^{t} g_{N}(s) \tilde{x}_{2}(s) d s \\
& +32 \sqrt{\frac{\omega_{2}}{N}} e^{32\left(\lambda_{1} \beta / \alpha+1\right)} \frac{\beta}{\alpha}\left(\lambda_{1}-1\right) t+8 e^{t \eta_{2}} \sqrt{\frac{\omega_{1} \lambda_{1}\left(\lambda_{1}-1\right)}{N\left(\lambda_{1}-\lambda_{2}\right)}} \\
& +8 \lambda_{2} \sqrt{\frac{\omega_{2}}{N}} e^{32\left(\lambda_{1} \beta / \alpha+1\right)} \int_{0}^{t} g_{N}(s) d s+\sqrt{\frac{\omega_{1}}{N\left(\lambda_{1}-\lambda_{2}\right)^{2}}} \log ^{1 / 2}\left(\frac{\lambda_{1}-1}{\lambda_{1}-\lambda_{2}}\right) e^{\omega_{1} / 8} x_{2}(t) e^{t \eta_{2}} .
\end{aligned}
$$

Let $t_{0}=t_{0}(N)=\lambda_{1}\left(\lambda_{1}-\lambda_{2}\right)^{-1} e^{\omega_{1} / 8}$. By Grönwall's lemma, for all $t \leq$ $t_{0}(N)$, on the event $t<T_{1} \wedge T_{2} \wedge T_{3}$,

$$
\begin{aligned}
g_{N}(t) & \leq\left(32 \sqrt{\frac{\omega_{2}}{N}} e^{32\left(\lambda_{1} \beta / \alpha+1\right)} \frac{\beta}{\alpha}\left(\lambda_{1}-1\right) t+8 e^{t \eta_{2}} \sqrt{\frac{\omega_{1} \lambda_{1}\left(\lambda_{1}-1\right)}{N\left(\lambda_{1}-\lambda_{2}\right)}}\right. \\
& \left.+\sqrt{\frac{\omega_{1}}{N\left(\lambda_{1}-\lambda_{2}\right)^{2}}} \log ^{1 / 2}\left(\frac{\lambda_{1}-1}{\lambda_{1}-\lambda_{2}}\right) e^{\omega_{1} / 8} x_{2}(t) e^{t \eta_{2}}\right) e^{H_{N}(t)}
\end{aligned}
$$

where

$$
\begin{aligned}
H_{N}(t) & =\lambda_{2} \int_{0}^{t}\left|\tilde{x}_{1}(s)\right| d s+\frac{4\left(\lambda_{1}-\lambda_{2}\right)}{\lambda_{1}-1} \int_{0}^{t} \tilde{x}_{2}(s) d s+8 \lambda_{2} t \sqrt{\frac{\omega_{2}}{N}} e^{32\left(\lambda_{1} \beta / \alpha+1\right)} \\
& \leq 16\left(1+\frac{\beta}{\alpha}\right)+\frac{8 \lambda_{1} \lambda_{2}}{\sqrt{N}\left(\lambda_{1}-\lambda_{2}\right)} \sqrt{\omega_{2}} e^{\omega_{1} / 8} e^{32\left(\lambda_{1} \beta / \alpha+1\right)} \\
& \leq 16\left(1+\frac{\beta}{\alpha}\right)+\frac{1}{\sqrt{N}\left(\lambda_{1}-\lambda_{2}\right)} \sqrt{\log \left(\frac{\lambda_{1}-1}{\lambda_{1}-\lambda_{2}}\right)} e^{\omega_{1} / 4} \\
& \leq 32\left(1+\frac{\beta}{\alpha}\right),
\end{aligned}
$$

for $N$ large enough, since

$$
N\left(\lambda_{1}-\lambda_{2}\right)^{2} / \log \left(\frac{\lambda_{1}-1}{\lambda_{1}-\lambda_{2}}\right) e^{\omega_{1} / 2} \rightarrow \infty .
$$


EXTINCTION TIME FOR THE WEAKER OF TWO COMPETING SIS EPIDEMICS 45

It follows that, for $t \leq t_{0}(N)$, on the event $t<T_{1} \wedge T_{2} \wedge T_{3}$, if $N$ is large enough, then

$$
\begin{aligned}
f_{N}(t) & \leq\left(32 \sqrt{\frac{\omega_{2}}{N}} e^{32\left(\lambda_{1} \beta / \alpha+1\right)} \frac{\beta}{\alpha}\left(\lambda_{1}-1\right) t e^{-t \eta_{2}}+8 \sqrt{\frac{\omega_{1} \lambda_{1}\left(\lambda_{1}-1\right)}{N\left(\lambda_{1}-\lambda_{2}\right)}}\right. \\
& \left.+\sqrt{\frac{\omega_{1}}{N\left(\lambda_{1}-\lambda_{2}\right)^{2}}} \log ^{1 / 2}\left(\frac{\lambda_{1}-1}{\lambda_{1}-\lambda_{2}}\right) e^{\omega_{1} / 8} x_{2}(t)\right) e^{32\left(1+\frac{\beta}{\alpha}\right)} .
\end{aligned}
$$

Now, using (19) and (20), it thus follows that, for $t \leq t_{0}(N)$, on the event $t<T_{1} \wedge T_{2} \wedge T_{3}$, for $N$ large enough,

$$
\begin{aligned}
f_{N}(t) \leq(128 & \frac{\alpha+\beta}{\alpha} \sqrt{\frac{\log \left(\lambda_{1}\left(\lambda_{1}-1\right) /\left(\lambda_{1}-\lambda_{2}\right)\right)+\omega_{1}}{N}} e^{32\left(\lambda_{1} \beta / \alpha+1\right)} \lambda_{1} x_{2}(t) t \\
& \left.+8 \sqrt{\frac{\omega_{1} \lambda_{1}\left(\lambda_{1}-1\right)}{N\left(\lambda_{1}-\lambda_{2}\right)}}+\sqrt{\frac{\omega_{1}}{N\left(\lambda_{1}-\lambda_{2}\right)^{2}}} \log ^{1 / 2}\left(\frac{\lambda_{1}-1}{\lambda_{1}-\lambda_{2}}\right) e^{\omega_{1} / 8} x_{2}(t)\right) e^{32\left(1+\frac{\beta}{\alpha}\right)},
\end{aligned}
$$

Now, for all $t \leq t_{0}(N)$ such that $t<T_{1} \wedge T_{2} \wedge T_{3}$, for $N$ large enough,

$$
f_{N}(t) \leq\left(2\left(\lambda_{1}-\lambda_{2}\right)^{-1} \log ^{1 / 2}\left(\frac{\lambda_{1}-1}{\lambda_{1}-\lambda_{2}}\right) e^{\omega_{1} / 8} x_{2}(t)+8 \sqrt{\frac{\lambda_{1}\left(\lambda_{1}-1\right)}{\lambda_{1}-\lambda_{2}}}\right) \sqrt{\frac{\omega_{1}}{N}} e^{32\left(1+\frac{\beta}{\alpha}\right)},
$$

Let $T_{4}$ be the infimum of times $t$ such that

$$
f_{N}(t)>\left(2\left(\lambda_{1}-\lambda_{2}\right)^{-1} \log ^{1 / 2}\left(\frac{\lambda_{1}-1}{\lambda_{1}-\lambda_{2}}\right) e^{\omega_{1} / 8} x_{2}(t)+8 \sqrt{\frac{\lambda_{1}\left(\lambda_{1}-1\right)}{\lambda_{1}-\lambda_{2}}}\right) \sqrt{\frac{\omega_{1}}{N}} e^{32\left(1+\frac{\beta}{\alpha}\right)} .
$$

By the above, and as in the proof of Lemma 14,

$\mathbb{P}\left(T_{4} \leq t_{0}\right) \leq \mathbb{P}\left(T_{1} \wedge T_{2} \wedge T_{3} \leq T_{4} \wedge t_{0}\right) \leq \mathbb{P}\left(T_{1} \leq t_{0}\right)+\mathbb{P}\left(T_{2} \leq T_{1} \wedge t_{0}\right)+\mathbb{P}\left(T_{3} \leq T_{4}\right)$.

By Lemma 14, $\mathbb{P}\left(T_{1} \leq t_{0}\right) \leq 12 e^{-\omega_{2} / 8}$. By Lemma 7 applied to $\left(\tilde{x}_{N}(t)\right)$, this time taking $\sigma=\left(\lambda_{1}-\lambda_{2}\right)^{-1}, K_{2}=4 \lambda_{1}\left(\lambda_{1}-1\right) / N\left(\lambda_{1}-\lambda_{2}\right)$, and $\eta=\eta_{2}$, $\mathbb{P}\left(T_{2} \leq T_{1} \wedge t_{0}\right) \leq 4 e^{-\omega_{1} / 8}$. Also, clearly, for $N$ large enough, $\mathbb{P}\left(T_{3} \leq T_{4}\right)=0$. It follows that $\mathbb{P}\left(T_{4} \leq t_{0}\right) \leq 16 e^{-\omega_{1} / 8}$, as required.

Proof of Theorem 4. Let

$t_{1}=t_{1}(N)=\left(\lambda_{1}-1\right)^{-1 / 2}\left(\lambda_{1}-\lambda_{2}\right)^{-1 / 2}=\frac{1}{\lambda_{1}-\lambda_{2}} \sqrt{\frac{\lambda_{1}-\lambda_{2}}{\lambda_{1}-1}}=\frac{1}{\lambda_{1}-1} \sqrt{\frac{\lambda_{1}-1}{\lambda_{1}-\lambda_{2}}}$.

By Lemma 13 with $\psi=\left(\lambda_{1}-1\right)^{1 / 2}\left(\lambda_{1}-\lambda_{2}\right)^{-1 / 2}$, for $N$ large enough,

$\mathbb{P}\left(\left|\frac{X_{N, 1}\left(t_{1}\right)}{X_{N, 2}\left(t_{1}\right)}-\frac{X_{N, 1}(0)}{X_{N, 2}(0)}\right|>2\left(\frac{\lambda_{1}-\lambda_{2}}{\lambda_{1}-1}\right)^{1 / 8}\right) \leq 4 e^{-\sqrt{N}\left(\lambda_{2}-1\right)}+e^{-\left(\frac{\lambda_{1}-1}{\lambda_{1}-\lambda_{2}}\right)^{1 / 8}}$.

Assume that $x_{N, 1}(0)+x_{N, 2}(0)>\left(\lambda_{1}-1\right) / \lambda_{1}$. Let $x=\min \left\{x_{N, 1}(0)+\right.$ $\left.x_{N, 2}(0), N^{-1}\left\lfloor N\left(\lambda_{2}-1\right)\left(N\left(\lambda_{2}-1\right)^{2}\right)^{1 / 8}\right\rfloor\right\}$, and let $T$ be the infimum of times $t$ such that $x_{N, 1}(t)+x_{N, 2}(t)=x$. Lemma 4.1 in Brightwell, House and Luczak (2017) is still valid for a supercritical stochastic SIS logistic epidemic, so by 
that lemma, combined with Lemma 10 in the present paper and Markov's inequality, for $N$ large enough,

$$
\mathbb{P}\left(x_{N, 1}\left(t_{1} / 4\right)+x_{N, 2}\left(t_{1} / 4\right)>x\right) \leq 2\left(N\left(\lambda_{2}-1\right)^{2}\right)^{-1 / 8},
$$

and so $T \leq t_{1} / 4$ with probability at least $1-2\left(N\left(\lambda_{2}-1\right)^{2}\right)^{-1 / 8}$.

Let $y(t)$ solve equation (16) with $\lambda=\lambda_{1}$ and $\mu=1, y(T)=x$, and let $z(t)$ solve equation (16) with $\lambda=\lambda_{2}$ and $\mu=1$, and $z(T)=x$. Let $Y_{N}(t)$ and $Z_{N}(t)$ be the corresponding stochastic SIS logistic epidemics satisfying $Y_{N}(T)=N x$ and $Z_{N}(T)=N x$ respectively. It is easily seen from (18) that, if $N$ is sufficiently large and $T \leq t_{1} / 4$, then $y\left(t_{1} / 2\right) \leq 2\left(\lambda_{1}-1\right) / \lambda_{1}$ and $z\left(t_{1} / 2\right) \leq 2\left(\lambda_{2}-1\right) / \lambda_{2}$. Furthermore, using (18) over the time-interval $\left[t_{1} / 2, t_{1}\right]$, we see that in that case

$$
\left|y\left(t_{1}\right)-\left(\lambda_{1}-1\right) / \lambda_{1}\right| \leq \frac{\lambda_{1}-1}{\lambda_{1}} e^{-\frac{1}{2} \sqrt{\frac{\lambda_{1}-1}{\lambda_{1}-\lambda_{2}}}},
$$

and

$$
\left|z\left(t_{1}\right)-\left(\lambda_{2}-1\right) / \lambda_{2}\right| \leq \frac{\lambda_{2}-1}{\lambda_{2}} e^{-\left(\lambda_{2}-1\right) t_{1} / 2} \leq \frac{\lambda_{2}-1}{\lambda_{2}} e^{-\frac{1}{4} \sqrt{\frac{\lambda_{1}-1}{\lambda_{1}-\lambda_{2}}}} .
$$

We will now apply Lemma 12 twice, both starting at time $T$, once with $\lambda=\lambda_{1}, \mu=1$, ending at time $\tau_{1}=\inf \left\{t \geq T: y(t) \leq 2\left(\lambda_{1}-1\right) / \lambda_{1}\right\}$, and the second time with $\lambda=\lambda_{2}, \mu=1$, ending at time $\tau_{2}=\inf \{t \geq T: z(t) \leq$ $\left.2\left(\lambda_{2}-1\right) / \lambda_{2}\right\}$. We further apply Lemma 11] twice, once to $Y_{N}(t)$, starting at time $\tau_{1}$, with $\lambda=\lambda_{1}, \mu=1$ and $\omega=\sqrt{N\left(\lambda_{1}-1\right)^{2}}$, and once to $Z_{N}(t)$, starting at time $\tau_{2}$, with $\lambda=\lambda_{2}, \mu=1$ and $\omega=\sqrt{N\left(\lambda_{2}-1\right)^{2}}$. Additionally applying Lemma 10, we see that, for $N$ sufficiently large,

$$
\begin{array}{r}
\mathbb{P}\left(\left|x_{N, 1}\left(t_{1}\right)+x_{N, 2}\left(t_{1}\right)-\frac{\lambda_{1}-1}{\lambda_{1}}\right|>6 e^{4} \frac{\left(N\left(\lambda_{1}-1\right)^{2}\right)^{1 / 4} \sqrt{\lambda_{1}+1}}{\sqrt{N \lambda_{2}}}+\lambda_{1}-\lambda_{2}\right) \\
\leq 12 e^{-\left(N\left(\lambda_{2}-1\right)^{2}\right)^{1 / 8} / 8}+2\left(N\left(\lambda_{2}-1\right)^{2}\right)^{-1 / 8} \leq 3\left(N\left(\lambda_{2}-1\right)^{2}\right)^{-1 / 8} .
\end{array}
$$

In particular, we see that with probability $1-\delta_{N}$ event $\mathcal{E}_{N}$ holds that $x_{N, 1}\left(t_{1}\right)+x_{N, 2}\left(t_{1}\right) \leq 2\left(\lambda_{1}-1\right) / \lambda_{1}, x_{N, 1}\left(t_{1}\right) / x_{N, 2}\left(t_{2}\right)=\alpha / \beta+\varepsilon_{N}$, where $\delta_{N}, \varepsilon_{N} \rightarrow 0$ as $N \rightarrow \infty$.

In the case when $x_{N, 1}(0)+x_{N, 2}(0)<\left(\lambda_{1}-1\right) / \lambda_{1}$ (this is only relevant when $\lambda_{1}$ is bounded away from 1 ), we can skip the first two phases and only use Lemma 11, We omit the details.

Let $\omega_{1}=\omega_{1}(N) \rightarrow \infty$ be such that $\omega_{1} \leq \log \left(\frac{\lambda_{1}-1}{\lambda_{1}-\lambda_{2}}\right)$ and let $\omega_{2}=$ $16 \log \left(\frac{\lambda_{1}-1}{\lambda_{1}-\lambda_{2}}\right)$. Let $t_{2}=t_{2}(N)=t_{1}(N)+\frac{\lambda_{1}}{\lambda_{1}-\lambda_{2}} e^{\omega_{1} / 8}$, and note that $t_{2}-t_{1} \leq$ $\left(\lambda_{1}-1\right)^{-1} e^{\omega_{2} / 8}$.

Consider solution $x(t)=\left(x_{1}(t), x_{2}(t)\right)^{T}$ to (11) subject to condition $x_{1}\left(t_{1}\right)=$ $\left.N^{-1} X_{N, 1}\left(t_{1}\right), x_{2}\left(t_{1}\right)=N^{-1} X_{N, 2}\left(t_{1}\right)\right)^{T}$. Let also $\tilde{x}(t)=\left(\tilde{x}_{1}(t), \tilde{x}_{2}(t)\right)^{T}$ be the corresponding solution to (9). By (19),

$$
x_{2}\left(t_{2}\right) \leq 4(\beta / \alpha) \frac{\lambda_{1}-1}{\lambda_{1}} e^{-\left(t_{2}-t_{1}\right)\left(\lambda_{1}-\lambda_{2}\right) / \lambda_{1}},
$$


EXTINCTION TIME FOR THE WEAKER OF TWO COMPETING SIS EPIDEMICS 47

and by (20)

$$
x_{2}\left(t_{2}\right) \geq \frac{1}{4} \frac{\beta}{\alpha+\beta}\left(\lambda_{1}-1\right) e^{-\left(t_{2}-t_{1}\right) \frac{\lambda_{1}-\lambda_{2}}{\lambda_{1}}} .
$$

for $N$ large enough. Note that $\omega_{1}$ can be chosen in such a way that $\left(\lambda_{1}-\right.$ $\left.\lambda_{2}\right)^{-1} x_{2}\left(t_{2}\right) \rightarrow 0$ : for instance, we choose $\omega_{1}$ satisfying $e^{\omega_{1} / 8}=\log \left(\frac{\lambda_{1}-1}{\lambda_{1}-\lambda_{2}}\right)+$ $\phi$ for a suitable $\phi=\phi(N) \rightarrow \infty$ such that $\phi \leq \log \left(\frac{\lambda_{1}-1}{\lambda_{1}-\lambda_{2}}\right)$. Since then $x_{2}\left(t_{2}\right) \geq \frac{1}{4} \frac{\beta}{\alpha+\beta}\left(\lambda_{1}-\lambda_{2}\right)^{2}\left(\lambda_{1}-1\right)^{-1}$, we further have $N x_{2}\left(t_{2}\right)\left(\lambda_{1}-\lambda_{2}\right) \rightarrow \infty$.

Note that, since $N\left(\lambda_{1}-\lambda_{2}\right)^{3}\left(\lambda_{1}-1\right)^{-1} \rightarrow \infty$, conditions of Lemmas 14 and 15 are satisfied. By Lemma 14 with $\omega=\omega_{2}$ and by Lemma 15 with the value of $\omega_{1}$ above, with $X_{N, 1}\left(t_{1}\right), X_{N, 2}\left(t_{1}\right)$ as initial values, with probability at least $1-16 e^{-\omega_{1} / 8}-12 e^{-\omega_{2} / 8}$, the event $\mathcal{E}\left(t_{2}\right)$ holds that

$$
\left|\tilde{x}_{N, 1}\left(t_{2}\right)-\tilde{x}_{1}\left(t_{2}\right)\right| \leq 8 \sqrt{\frac{\omega_{2}}{N}} e^{32\left(\lambda_{1} \beta / \alpha+1\right)},
$$

and

$$
\begin{aligned}
& \left|\tilde{x}_{N, 2}\left(t_{2}\right)-\tilde{x}_{2}\left(t_{2}\right)\right| \\
& \quad \leq\left[2\left(\lambda_{1}-\lambda_{2}\right)^{-1} \log ^{1 / 2}\left(\frac{\lambda_{1}-1}{\lambda_{1}-\lambda_{2}}\right) e^{\omega_{1} / 8} x_{2}\left(t_{2}\right)+8 \sqrt{\frac{\lambda_{1}\left(\lambda_{1}-1\right)}{\lambda_{1}-\lambda_{2}}}\right] \sqrt{\frac{\omega_{1}}{N}} e^{32(1+\beta / \alpha)} .
\end{aligned}
$$

Hence also, for $N$ large enough, on $\mathcal{E}\left(t_{2}\right)$,

$$
\begin{aligned}
& \left|x_{N, 1}\left(t_{2}\right)-\left(\lambda_{1}-1\right) / \lambda_{1}\right| \\
& \quad \leq\left[2\left(\lambda_{1}-\lambda_{2}\right)^{-1} \log ^{1 / 2}\left(\frac{\lambda_{1}-1}{\lambda_{1}-\lambda_{2}}\right) e^{\omega_{1} / 8} x_{2}\left(t_{2}\right)+10 \sqrt{\frac{\lambda_{1}\left(\lambda_{1}-1\right)}{\lambda_{1}-\lambda_{2}}}\right] \sqrt{\frac{\omega_{1}}{N}} e^{32(1+\beta / \alpha)} \\
& \quad+8 \frac{\beta}{\alpha} \frac{\lambda_{1}-1}{\lambda_{1}} e^{-\left(t_{2}-t_{1}\right)\left(\lambda_{1}-\lambda_{2}\right) / \lambda_{1}} .
\end{aligned}
$$

Note that

$$
N\left(\lambda_{1}-\lambda_{2}\right)^{3}\left(\lambda_{1}-1\right)^{-1} / \log \log \left(N\left(\lambda_{1}-\lambda_{2}\right)^{2}\right) \rightarrow \infty
$$

implies that

$$
N\left(\lambda_{1}-\lambda_{2}\right)^{3}\left(\lambda_{1}-1\right)^{-1} / \log \log \left(\frac{\lambda_{1}-1}{\lambda_{1}-\lambda_{2}}\right) \rightarrow \infty,
$$

and so we can choose $\phi$ so that

$$
N\left(\lambda_{1}-\lambda_{2}\right)^{3}\left(\lambda_{1}-1\right)^{-1} / \omega_{1} e^{2 \phi} \rightarrow \infty .
$$

With this choice of $\phi$, it follows that

$$
\left(\lambda_{1}-1\right) e^{-\left(t_{2}-t_{1}\right) \frac{\lambda_{1}-\lambda_{2}}{\lambda_{1}}} \gg \sqrt{\frac{\lambda_{1}-1}{\lambda_{1}-\lambda_{2}}} \sqrt{\frac{\omega_{1}}{N}},
$$

and so, on the event $\mathcal{E}\left(t_{2}\right)$, both $x_{N, 1}\left(t_{2}\right)$ and $x_{N, 2}\left(t_{2}\right)$ are concentrated around $\left(\lambda_{1}-1\right) / \lambda_{1}$ and $x_{2}\left(t_{2}\right)$ respectively, $x_{N, 1}\left(t_{2}\right)$ with error of size $o\left(\lambda_{1}-\right.$ 
$\left.\lambda_{2}\right)$ and $x_{N, 2}\left(t_{2}\right)$ with error of size $o\left(x_{2}\left(t_{2}\right)\right)=o\left(\lambda_{1}-\lambda_{2}\right)$. Also, $\mathbb{P}\left(\mathcal{E}\left(t_{2}\right)\right) \rightarrow 1$ as $N \rightarrow \infty$.

Let

$$
t_{3}=t_{2}+\frac{10 \lambda_{1}}{\lambda_{1}-\lambda_{2}} \log \left(N\left(\lambda_{1}-\lambda_{2}\right)^{2}\right) \leq t_{1}+\left(\lambda_{1}-1\right)^{-1} e^{\omega_{3} / 8}
$$

where

$$
\omega_{3}=32 \log \left(\frac{\lambda_{1}-1}{\lambda_{1}-\lambda_{2}}\right)+32 \log \log \left(N\left(\lambda_{1}-\lambda_{2}\right)^{2}\right) .
$$

Also,

$$
t_{3}-t_{1} \leq \frac{\lambda_{1}}{\lambda_{1}-\lambda_{2}} e^{\omega_{4} / 8}
$$

where

$$
\omega_{4}=16 \log \log \left(\frac{\lambda_{1}-1}{\lambda_{1}-\lambda_{2}}\right)+2 \log \log \left(N\left(\lambda_{1}-\lambda_{2}\right)^{2}\right) .
$$

Note that $\omega_{3}$ and $\omega_{4}$ satisfy conditions of Lemmas 14 and 15 respectively, so we can apply these Lemmas on the interval $\left[t_{1}, t_{3}\right]$.

For $t \geq t_{2}$, let $\tilde{\mathcal{E}}(t)$ be the event that, for all $s \in\left[t_{2}, t\right]$,

$$
\begin{aligned}
& \left|x_{N, 1}(s)-\left(\lambda_{1}-1\right) / \lambda_{1}\right| \\
& \quad \leq\left[4\left(\lambda_{1}-\lambda_{2}\right)^{-1}\left(\frac{\lambda_{1}-1}{\lambda_{1}-\lambda_{2}}\right)^{1 / 4} x_{2}\left(t_{2}\right)+20 \sqrt{\frac{\lambda_{1}\left(\lambda_{1}-1\right)}{\lambda_{1}-\lambda_{2}}}\right] \sqrt{\frac{\omega_{3}}{N}} e^{32(1+\beta / \alpha)} \\
& \quad+16 \frac{\beta}{\alpha} \frac{\lambda_{1}-1}{\lambda_{1}} e^{-\left(t_{2}-t_{1}\right)\left(\lambda_{1}-\lambda_{2}\right) / \lambda_{1}} .
\end{aligned}
$$

and $x_{N, 2}(s) \leq 2 x_{N, 2}\left(t_{2}\right)$. Note that on the event $\tilde{\mathcal{E}}_{t}$, for all $t_{2} \leq s \leq t$, $x_{N, 1}(s)$ is concentrated around $\left(\lambda_{1}-1\right) / \lambda_{1}$ with an $o\left(\lambda_{1}-\lambda_{2}\right)$ error.

By Lemmas 14 and 15, $\mathbb{P}\left(\tilde{\mathcal{E}}\left(t_{3}\right)\right) \rightarrow 1$ as $N \rightarrow \infty$.

On the event $\mathcal{E}\left(t_{2}\right)$, using a standard argument similar to the proof of Lemma 8 and the proof of Lemma 2.1 in Brightwell, House, and Luczak (2018), we couple the subsequent evolution of $X_{N, 2}(t)$ with two linear birthand-death chains, each with birth rate $\frac{\lambda_{2}}{\lambda_{1}}+o\left(\lambda_{1}-\lambda_{2}\right)$, and death rate 1 , so as to sandwich it between two such chains. The next event after time $t \geq t_{2}$ in each of the three chains can be coupled together, as long as event $\tilde{\mathcal{E}}(t)$ holds. Extinction happens by time $t_{3}$ with high probability, since the length of the final phase is, with high probability,

$$
\begin{array}{r}
\frac{\lambda_{1}}{\lambda_{1}-\lambda_{2}}\left(\log N+\log x_{2}\left(t_{2}\right)+\log \frac{\lambda_{1}-\lambda_{2}}{\lambda_{1}}+o(1)+G_{N}\right) \\
=\frac{\lambda_{1}}{\lambda_{1}-\lambda_{2}}\left(\log N\left(\lambda_{1}-\lambda_{2}\right)^{2}-\phi(N)+O(1)\right),
\end{array}
$$

where $G_{N}$ converges to a Gumbel random variable $G$ as $N \rightarrow \infty$.

The length of the 'fluid-limit' phase can be expressed as

$$
\frac{\lambda_{2}}{\lambda_{1}-\lambda_{2}} \log \left(x_{1}\left(t_{2}\right) / x_{1}\left(t_{1}\right)\right)-\frac{\lambda_{1}}{\lambda_{1}-\lambda_{2}} \log \left(x_{2}\left(t_{2}\right) / x_{2}\left(t_{1}\right)\right)
$$


and the length of the first phase is $t_{1}=\left(\lambda_{1}-\lambda_{2}\right)^{-1} \sqrt{\left(\lambda_{1}-\lambda_{2}\right) /\left(\lambda_{1}-1\right)}=$ $o\left(\left(\lambda_{1}-\lambda_{2}\right)^{-1}\right)$.

Hence, using the fact that $\left(\lambda_{1}-\lambda_{2}\right) \log \left(\lambda_{1}-1\right) \rightarrow 0$, the total time to extinction is, with high probability,

$$
\frac{\lambda_{1}}{\lambda_{1}-\lambda_{2}}\left(\log \left(\frac{N\left(\lambda_{1}-1\right)\left(\lambda_{1}-\lambda_{2}\right) \beta}{\lambda_{1}^{2} \alpha}+o(1)+G_{N}\right)\right),
$$

thus proving Theorem 4

7.1. Relaxing the assumption on separation from criticality. As stated above, we believe Theorem 4 is in fact valid under the weaker condition $N\left(\lambda_{1}-\lambda_{2}\right)^{2} \rightarrow \infty$ (still assuming $\mu_{1}=\mu_{2}=1$ and $\left(\lambda_{1}-\lambda_{2}\right)\left(\lambda_{1}-1\right)^{-1} \rightarrow$ $\infty)$. Here is a sketch of how one might go about proving such an extension. The differential equation approximation phase can be split into a number of subphases, each corresponding to a refined version of Lemma 15 with a smaller value of $x_{N, 2}(0)$ and thus a smaller bound on the quadratic variation of the martingale term. Roughly speaking the first subphase would have $x_{N, 2}(0)$ of order $\lambda_{1}-1$ and the martingale quadratic variation $N^{-1 / 2}\left(\lambda_{1}-\lambda_{2}\right)^{-1 / 2}\left(\lambda_{1}-1\right)^{1 / 2}$. The first subphase would last until $x_{N, 2}(t)$ is of size about $N^{-1 / 2}\left(\lambda_{1}-\lambda_{2}\right)^{-1 / 2}\left(\lambda_{1}-1\right)^{1 / 2} \omega^{3 / 4}$, for a suitable $\omega(N) \rightarrow \infty$, and would thus take time just slightly less than $\left(\lambda_{1}-\right.$ $\left.\lambda_{2}\right)^{-1} \log \sqrt{N\left(\lambda_{1}-1\right)\left(\lambda_{1}-\lambda_{2}\right)}$. The second subphase would have $x_{N, 2}(0)$ of order $N^{-1 / 2}\left(\lambda_{1}-\lambda_{2}\right)^{-1 / 2}\left(\lambda_{1}-1\right)^{1 / 2} \omega^{3 / 4}$ and the martingale quadratic variation $N^{-3 / 4}\left(\lambda_{1}-\lambda_{2}\right)^{-3 / 4}\left(\lambda_{1}-1\right)^{1 / 4} \omega^{3 / 8}$. It would last until $x_{N, 2}(t)$ is of size about $N^{-3 / 4}\left(\lambda_{1}-\lambda_{2}\right)^{-3 / 4}\left(\lambda_{1}-1\right)^{1 / 4} \omega^{15 / 16}$, and would thus take time just slightly less than $\left(\lambda_{1}-\lambda_{2}\right)^{-1} \log \left(N\left(\lambda_{1}-1\right)\left(\lambda_{1}-\lambda_{2}\right)\right)^{1 / 4}$. The third subphase would have $x_{N, 2}(0)$ of order $N^{-3 / 4}\left(\lambda_{1}-\lambda_{2}\right)^{-3 / 4}\left(\lambda_{1}-1\right)^{1 / 4} \omega^{15 / 16}$ and the martingale quadratic variation $N^{-7 / 8}\left(\lambda_{1}-\lambda_{2}\right)^{-7 / 8}\left(\lambda_{1}-1\right)^{1 / 8} \omega^{15 / 32}$. It would last until $x_{N, 2}(t)$ is of size about $N^{-7 / 8}\left(\lambda_{1}-\lambda_{2}\right)^{-7 / 8}\left(\lambda_{1}-1\right)^{1 / 8} \omega^{63 / 64}$, for a suitable $\omega(N) \rightarrow \infty$, and would thus take time just slightly less than $\left(\lambda_{1}-\lambda_{2}\right)^{-1} \log \left(N\left(\lambda_{1}-1\right)\left(\lambda_{1}-\lambda_{2}\right)\right)^{1 / 8}$. And, in principle, one should be able to carry on this process. The phases would be joined together using the end value of $x_{N, 2}(t)$ from the previous phase as initial condition for the differential equation in the next phase, and the various deterministic solutions with different random initial conditions would become closer and closer together over time.

As many phases would be used as needed to 'reach' $x_{N, 2}(t)$ of order $o\left(\lambda_{1}-\right.$ $\left.\lambda_{2}\right)$, while keeping the deviation smaller than the mean. Since the 'limiting' quadratic variation in the above process is $N^{-1}\left(\lambda_{1}-\lambda_{2}\right)^{-1}$, this should in principle be possible as long as $N\left(\lambda_{1}-\lambda_{2}\right)^{2} \rightarrow \infty$, and as $N\left(\lambda_{1}-\lambda_{2}\right)^{2}$ tends to infinity more and more slowly, the time spent in the differential equation phase becomes closer and closer to $\left(\lambda_{1}-\lambda_{2}\right)^{-1} \log \left(N\left(\lambda_{1}-1\right)\left(\lambda_{1}-\lambda_{2}\right)\right)$.

After the condition $N\left(\lambda_{1}-\lambda_{2}\right)^{2} \rightarrow \infty$ fails, one can still carry out the differential equation phase from the time when $x_{N, 2}$ if of the order $\lambda_{1}-1$ through the various phases until it is of the order about $N^{-1}\left(\lambda_{1}-\lambda_{2}\right)^{-1}$, 
which takes time of the order about $\left(\lambda_{1}-\lambda_{2}\right)^{-1} \log \left(N\left(\lambda_{1}-1\right)\left(\lambda_{1}-\lambda_{2}\right)\right)$. After that, the fluctuations dominate, and the remaining time is about $\left(\lambda_{1}-\lambda_{2}\right)^{-2}$ steps, translating to a time of order $N^{-1}\left(\lambda_{1}-\lambda_{2}\right)^{-2}=o\left(\left(\lambda_{1}-\lambda_{2}\right)^{-1}\right)$.

A differential equation approximation phase is possible as long as the initial quadratic variation $N^{-1 / 2}\left(\lambda_{1}-\lambda_{2}\right)^{-1 / 2}\left(\lambda_{1}-1\right)^{1 / 2}$ is $o\left(\lambda_{1}-1\right)$, which is as long as $N\left(\lambda_{1}-1\right)\left(\lambda_{1}-\lambda_{2}\right) \rightarrow \infty$.

This would join up our result nicely with that of Kogan et al. (2014), showing that when the two basic reproductive ratios are equal, then the time to extinction is of order $N$.

We hope all the details above can be filled in to yield a complete proof, but we leave this till the next paper.

\section{ACKNowledgement}

The authors are grateful to Graham Brightwell for helpful comments that inspired Lemma 15, and as a result helped us achieve an improved understanding of near-critical phenomena in this model.

\section{REFERENCES}

Anderson RM, May RM (1996) The population biology of the interaction between HIV-1 and HIV-2; coexistence or competitive exclusion? AIDS 10:1663-1673

Andersson H, Djehiche B (1998) A threshold limit theorem for the stochastic logistic epidemic. J Appl Probab 35:662-670

Andreassen V, Lin J, Levin SA (1997) The dynamics of cocirculating influenza strains conferring partial cross-immunity. J Math Biol 35:825-842

Antia R, Regoes RR, Koella JC, Bergstrom CT (2003) The role of evolution in the emergence of infectious diseases. Nature 426:658-661

Arinaminpathy N, McLean AR (2009) Evolution and emergence of novel human infections. P Roy Soc Lond B Bio 276:3937-3943

Bahl J, Vijaykrishna D, Holmes EC, Smith GJD, Guan Y (2009) Gene flow and competitive exclusion of avian influenza $A$ virus in natural reservoir hosts. Virology 390:289-297

Barbour AD, Hamza K, Kaspi H, Klebaner FC (2015) Escape from the boundary in Markov population processes. Adv Appl Probab 47:11901211

Barbour AD, Luczak MJ (2012) A law of large numbers approximation for Markov population processes with countably many types. Probab Theory Rel 153:727-757

Brightwell GR, House T, Luczak MJ (2018) Extinction times in the subcritical stochastic SIS logistic epidemic, to appear in J Math Biol. arXiv:1312.7449

Brightwell GR, Luczak MJ (2012) Vertices of high degree in the preferential attachment tree. Electron J Probab 17 
Castillo-Chavez C, Huang H, Li J (1999) Competitive exclusion and coexistence of multiple strains in an SIS STD model. SIAM J Appl Math 59:1790-1811

Cattiaux P, Méléard S (2010) Competitive or weak cooperative stochastic Lotka-Volterra systems conditioned on non-extinction. J Math Biol 6:797829

Darling RWR, Norris JR (2008) Differential equation approximation for Markov chains. Probab Surveys 5:37-79

Domingo E, Escarmis C, Sevilla N, Moya A, Elena SF, Quer J, Novella IS, Holland JJ (1996) Basic concepts in RNA virus evolution. FASEB J 10:859-864

Feng Z, Velasco-Hernandez JX (1997) Competitive exclusion in a vector-host model for dengue fever. J Math Biol 35:523-544

Gart JJ, De Vries JL (1966) The mathematical analysis of concurrent epidemics of yaws and chickenpox. J Hyg-Camb 64:431-439

Humplik J, Hill AL, Nowak MA (2014) Evolutionary dynamics of infectious diseases in finite populations. J Theor Biol 360:149-162

Kirupaharan N, Allen LJS (2004) Coexistence of multiple pathogen strains in stochastic epidemic models with density-dependent mortality. B Math Biol 66:841-864

Kogan O, Khasin M, Meerson B, Schneider D, Myers CR (2014) Twostrain competition in quasineutral stochastic disease dynamics. Phys Rev E 90:042149

Kurtz TG (1970) Solutions of ordinary differential equations as limits of pure jump Markov processes. J Appl Probab 7:49-58

Leventhal GE, Hill AL, Nowak MA, Bonhoeffer S (2015) Evolution and emergence of infectious diseases in theoretical and real-world networks. Nat Commun 6:6101

Levin SA (1970) Community equilibria and stability, and an extension of the competitive exclusion principle. Am Nat 104: 413-423

Lotka AJ (1925) Elements of physical biology. Williams and Wilkins.

Moran PAP (1958) Random processes in genetics. Math Proc Cambridge 54:60-71

Moya A, Holmes EC, Gonzáles-Candelas F (2004) The population genetics and evolutionary epidemiology of RNA virus. Nat Rev Microbiol 2:279288

Nåsell I (2011) Extinction and Quasi-stationarity in the Stochastic Logistic SIS Model. Lecture Notes in Mathematics 2022. Springer-Verlag, Berlin, Heidelberg

Parsons TL, Quince C (2007a) Fixation and haploid populations exhibiting density dependence i: the non-neutral case. Theor Popul Biol 72:121-135

Parsons TL, Quince C (2007b) Fixation and haploid populations exhibiting density dependence ii: the quasi-neutral case. Theor Popul Biol 72:468479 
Parsons TL, Quince C, Plotkin JB (2008) Absorption and fixation times for neutral and quasi-neutral populations with density dependence. Theor Popul Biol 74:302-310

Renshaw E (2011) Stochastic Population Processes. Oxford University Press Schrag SJ, Wiener P (1995) Emerging infectious disease: what are the relative roles of ecology and evolution? Trends Ecol Evol 10:319-324

Thorisson H (2000) Coupling, Stationarity and Regeneration. Probability and Applications. Springer-Verlag, New York.

Volterra V (1931) Leçons sur la Théorie Mathématique de la Lutte pour la Vie. Gautier-Villars.

Walter W (1998) Ordinary Differential Equations. Graduate Texts in Mathematics. Springer-Verlag, New York.

Zeeman ML (1995) Extinction in competitive Lotka-Volterra systems. P Am Math Soc 123:87-96

Pontificia Universidad Católica de Valparaiso.

School of Mathematics and Statistics, University of Melbourne 